\title{
LIPIDS AND THYROID HORMONES
}

\author{
FreDERIC L. HoCH*
}

Departments of Internal Medicine and Biological Chemistry, The University of Michigan Medical School, Ann Arbor, Michigan 48109, U.S.A.

\section{CONTENTS}

Abbreviations

I. Thyroid Hormone Mechanisms

A. Calorigenic and anabolic effects

B. Thyroid hormone entry into cells

II. LIPID MetabolisM

A. Cell lipid composition

B. Fatty acid synthesis

1. Protein phosphorylation-dephosphorylation $\quad 206$

2. Gene expression

C. NADPH generation and oxidation

1. Cytoplasm: NADP ${ }^{+} \rightarrow$ NADPH

2. Mitochondria: NADP $^{+}+\mathrm{NADH} \rightarrow \mathrm{NADPH}+\mathrm{NAD}^{+}$

3. Microsomes: NADPH $\rightarrow$ NADP $^{+}$

D. Fatty acid activation

E. Glycerolipid synthesis

F. Lipolysis

G. Ketogenesis

H. Cholesterol metabolism

1. Intracellular cholesterol metabolism

2. Cholesterol transport $\quad 220$

I. Fatty acid desaturation $\quad 221$

1. Anuran metamorphosis 223

J. Fatty acid oxidation $\quad 224$

$\begin{array}{ll}\text { K. Prostanoid synthesis } & 227\end{array}$

L. Sulfolipid synthesis $\quad 227$

227
2.27

III. Cell Membrane and Associated Systems

A. Lipid composition and physical properties $\quad 228$

B. cAMP synthesis and hydrolysis $\quad 229$

1. Adenylate cyclase $\quad 229$

2. Cyclic nucleotide phosphodiesterases

C. $\mathrm{Ca}^{2+}$-ATPases 233

D. $\mathrm{Na}^{+} \mathrm{K}^{+}$-ATPase $\quad 235$

E. Plasma Membrane Permeability $\quad 239$

1. $\mathrm{Na}^{+}, \mathrm{K}^{+}$-Cotransporters 239

2. $\mathrm{Na}^{+}-\mathrm{H}^{+}$Antiporter $\quad 240$

IV. Mitochondrial Oxidative Phosphorylation $\quad 241$

A. State 4 respiration in liver mitochondria

B. State 3 respiration in liver mitochondria $\quad 248$

$\begin{array}{ll}\text { 1. ADP/ATP carrier } & 249\end{array}$

2. Substrate carriers $\quad 250$

3. Cytochrome $b c_{1}$ segment 250

4. Cytochrome oxidase $\quad 251$

5. $P_{\mathrm{i}}$ transporter 251

6. Substrate dehydrogenases 251

7. ATP-synthetase 252

8. $\mathrm{Ca}^{2+}$-transporter 252

9. Pyridine nucleotide transhydrogenase

10. Repetitive additions of ADP $\quad 254$

C. Liver mitochondria of fetal and neonatal rats

D. Heart and brain mitochondria $\quad 255$

E. Essential fatty acid deficiency

$\begin{array}{lr}\text { V. Perspective } & 259\end{array}$

ACKNOWLEDGEMENTS $\quad 259$

$\begin{array}{lr}\text { REFERENCES } & 259\end{array}$

T3-triiodothyronine

ABBREVIATIONS

T4-thyroxine

EFA-essential fatty acids

FFA-free fatty acids

*Mailing address: 3455 Woodland Road, Ann Arbor, MI 48104, U.S.A. 
UFA-unsaturated fatty acyls

MUFA-monounsaturated fatty acyls

PUFA-polyunsaturated fatty acyls

HMGR $-\beta$-hydroxy $\beta$-methylglutaryl-CoA reductase

CPT-carnitine palmitoyl transferase

TH, TH-pyridine nucleotide transhydrogenase, energy-independent or -dependent

UI- unsaturation index [ $\Sigma(\%$ fatty acyl group $\times$ number of unsaturated bonds $)]$

$[\Psi]-$ concentration of $\Psi$

\section{THYROID HORMONE MECHANISMS}

The availability of thyroid hormones affects reactions in almost all pathways of lipid metabolism, as has been documented in previous reviews. ${ }^{23,226,231,233,477,641}$ Changes in lipid metabolism are only a portion of the diverse, pleiotypic effects of altered thyroid states on cell metabolism. Attempts have been made to reduce this diversity into one or a few common initial events. Such an event should include the specific binding of L-triiodothyronine (LT3) or thyroxine (LT4) at a cellular locus that exists only in thyroid-sensitive cells. Integrated overviews of thyroid regulation have been based on the consequences of receptor occupancy only in nuclei, with subsequent gene expression; ${ }^{125,451-454,599}$ in mitochondria, with subsequent modulation of cell energy metabolism; ${ }^{226,231.233}$ in both nuclei and mitochondria; ${ }^{577}$ and in nuclei, mitochondria, plasma membranes, and perhaps cytosol. ${ }^{412}$ Evidence is reviewed here that thyroid hormones act at nuclear receptors to express genes for a few lipogenic enzymes and perhaps their modifiers, and that ensuing changes in membrane lipid compositions mediate processes that underlie part of the pleiotypy. Lipids of membranes are considered both as regulating membrane-dependency that modulates protein enzyme-, transporter- or receptor-mediated activities, and as permitting unmediated cation leakage. Pertinent thyroid hormone mechanisms are first examined in general terms, then empirical observations of thyroid influence over lipid composition, and mechanisms whereby thyroid deficiency or administration regulates reactions in lipid metabolism. Effects of hormone-dependent lipid metabolism on membrane receptors, transporters, and enzymes are described in Sections III and IV.

\section{A. Calorigenic and Anabolic Effects}

Like other hormones, $\mathrm{T} 3$ and $\mathrm{T} 4$ are necessary intercellular messengers that coordinate cellular metabolic adaptations ${ }^{447}$ to a constantly changing external and internal environment. Three general coordinative roles can be distinguished for thyroid hormones. First, thyroid accelerates, and thyroid deficiency slows, catabolic, exergonic oxidative reactions in most cells. Thus, an appropriate level of thyroid hormone is necessary for an optimal rate of free energy liberation. Energy utilization by the ubiquitous $\mathrm{Na}^{+} \mathrm{K}^{+}$-ATPase is also thyroid-dependent, and is proposed to determine calorigenic rates (see Section III.D). Thyroid up-regulates lipid catabolism. Lipolysis of stored triglycerides and oxidation of fatty acids support increased calorigenesis. Second, thyroid hormones simultaneously exert major anabolic effects; lipogenesis is stimulated, which seems paradoxical for the calorigenic role of the hormones. In thyrotoxic subjects, the energetically wasteful combination of lipid synthesis and oxidation has been thought to act as a futile cycle to promote heat production. However, since hypothyroidism slows both synthesis and oxidation, normal $\mathrm{T} 3$ and $\mathrm{T} 4$ concentrations must also stimulate both processes constructively. This is seen in the requirement for thyroid hormones for growth and development in the mammalian fetus and neonate. I propose that thyroid-stimulated fatty acid synthesis and desaturations regulate mitochondrial oxidations via altered membrane fatty acyl compositions. Third, thyroid hormones have a permissive function, being necessary for and/or synergistic with actions of other hormones, e.g. lipolytic hormones, insulin, cortisol (in increasing mRNA synthesis), and testosterone (in inducing liver $\alpha_{2 U^{-}}$ globulin). The increase in scope of controls through permissive actions contributes to the synchronization of cell metabolism for whole-body adaptations, and is discussed in the next two sections. 
What organismic purpose is served when thyroid hormones stimulate both lipogenesis and fatty acid oxidation? Two are discussed in subsequent sections. One is calorigenic, in a futile cycle. The other is regulatory, the control of concentrations of specific metabolites through differential effects on both synthesis and further degradation. Some of these metabolites are effectors and also expand the hormone signal, e.g. cholesterol, cAMP, and membrane fatty acyl groups.

\section{B. Thyroid Hormone Entry into Cells}

Thyroid hormones act by binding to stereospecific, high-affinity, limited-capacity protein sites that impart a message when occupied that in turn alters cell metabolism. Sites with such properties are 'receptors', although that term is often used for just binding sites. Reasons to believe that the lipophilic T3 or T4 molecules themselves do not alter mitochondrial membrane permeability are discussed in Section IV. Thyroid hormone receptors exist in plasma membranes, ${ }^{455}$ cytosol, ${ }^{26}$ endoplasmic reticulum, ${ }^{84,85}$ mitochondrial inner membranes (Ref. 577, but see Ref. 189), and nuclei. Injected labeled hormone reaches all these sites within minutes (see Ref. 116). Receptor occupancy promotes gene expression in nuclei (see Section II.D), possibly oxidative phosphorylation in mitochondria (see Section IV), T3 and 2-deoxyglucose uptake, and $\mathrm{Ca}^{2+}$-ATPase activity in selected plasma membranes (see Section III.E.1), and T3 transport in cytosol.

Several sets of thyroid hormone-binding 'receptors' are described. 'The' nuclear receptor in all vertebrates that circulate $\mathrm{T} 3$ is a $50.5 \mathrm{kDa}$ protein, and its occupancy initiates thyroid action. ${ }^{454} \mathrm{~A}$ second 'receptor' is a common, abundant, LT3-binding protein of molecular mass $\sim 55 \mathrm{kDa}$ that is found in membranes of the endoplasmic reticulum and their continuations into the nuclear envelope in all lines of cultured cells examined. ${ }^{84,85}$ This protein is present in all tissues of an adult monkey, even those not thyroid-responsive, unlike the nuclear $50.5 \mathrm{kDa}$ receptor. In cultured cells of rat pituitary tumor $\left(\mathrm{GH}_{3}\right), 48-\mathrm{hr}$ incubation with $\mathrm{T} 3$ decreases the number of $55 \mathrm{kDa}$ protein molecules by specifically accelerating their degradation, but without changing their initial rate of synthesis or the amount of p55-coding mRNA. ${ }^{46 a}$ Thus, T3 regulates at a post-translational level. A similar mechanism of down-regulation of the $50.5 \mathrm{kDa}$ nuclear receptor operates in $\mathrm{GH}_{1}$ cells after incubation with T3 for $24 \mathrm{hr}$, but on briefer exposure T3 inhibits synthesis. ${ }^{521}$ Since T3 does not down-regulate liver T3-receptors in vivo, ${ }^{521}$ the susceptibility of the $55 \mathrm{kDa}$ protein to T3 control does not necessarily make it a 'receptor'. The sequence of the $55 \mathrm{kDa}$ protein is not similar to that of human plasma thyroid-binding prealbumin or globulin..$^{526,628}$ However, a brief note says that the coding region of the $55 \mathrm{kDa}$ protein has an $85 \%$ and $98 \%$ sequence homology to the endoplasmic reticulum enzymes protein disulfide isomerase and the $\beta$-subunit of prolyl 4-hydroxylase, respectively. ${ }^{85}$ It is not stated that the $55 \mathrm{kDa}$ protein has these enzymatic activities, but the $\beta$-subunit has the same disulfide isomerase activity in vitro as the disulfide isomerase itself, and monoclonal antibody to this subunit inhibits isomerase activity. ${ }^{476}$ The $\beta$-subunit is present in many cells in great excess over the active hydroxylase $\alpha \beta$-tetramers.

A third 'receptor' group comprises nuclear proteins, encoded by the c-erb-A gene, that are present in small amounts in many adult and embryonic cells. They have the binding properties of the nuclear T3-receptor although their functional capacities are not as yet described; weights are 46,52 , and $55 \mathrm{kDa}$; sequences of two cellular c-er $b-A$ proteins are dissimilar to that of the nuclear $55 \mathrm{kDa}$ protein. ${ }^{526,628} \mathrm{~A}$ viral counterpart encoded by the $\mathrm{v}$-erb- $A$ oncogene does not bind $\mathrm{T} 3$ similarly. A high degree of sequence homology between c-erb- $A$ protein and human glucocorticoid- and estradiol-receptor proteins suggests that all three may have evolved from a primordial receptor gene. ${ }^{630}$ All have a cysteine-rich region thought to bind DNA; the protein disulfide isomerase that resembles the nuclear $55 \mathrm{kDa}$ protein also has a cysteine-rich sequence in the active site. ${ }^{127}$

A fourth set of 'receptor' proteins with high affinity for thyroid hormones is identified in nuclei of tissues that do not contain the nuclear $55 \mathrm{kDa}$ protein and do not respond to administered hormone by increasing their respiration or lipogenesis. One is in the adult 
rat brain but not in liver, and is proposed to be one of many receptors that account for the diversity of thyroid hormone effects. ${ }^{605}$ Another is in testis. ${ }^{29}$ These binders await certification as receptors. Are the criteria for defining a receptor molecule by its binding properties to thyroid hormones sufficiently rigorous to exclude other lipophilic proteins found in membranes? A similar question applies to the identification of the mitochondrial T3-receptor as the ADP/ATP carrier (see Section IV.B.1).

Thyroid entry into cells is apparently membrane-mediated, being saturable, specific, and sensitive to temperature and to protein- and lipid-modifications. ${ }^{492}$ Plasma membrane receptors may be involved in T3 entry into cells. Triiodothyronine enters the cell across the plasma membrane via an energy-dependent transporter system. ${ }^{124,321,492}$ Extranuclear proteins bind $96 \%$ of hepatocyte $\mathrm{T} 3$; the minor fraction of $\mathrm{T} 3$ in hepatic nuclei plays a major role in promoting gene expression of lipogenic enzymes. In a rat pituitary tumor line, cytosol proteins compete less and nuclei contain $44 \%$ of cell T3. ${ }^{156}$ Recent evidence shows that another energy-dependent, stereospecific system carries LT3 across the hepatic cytosol to the nuclear binding sites; ${ }^{404,452}$ it accounts for the in situ nuclear LT3 content being 100 times greater than would be predicted from affinity constants of isolated nuclei or solubilized receptors. Free LT3 does bind to its nuclear receptor, however, unlike the steroids which must complex with a cytoplasmic receptor before they bind and act on nuclear components. The absence of reports that incubation of nuclei with $\mathrm{T} 3$ in vitro stimulates nuclear RNA polymerases therefore remains puzzling-unless it is an LT3-protein complex that actually binds to nuclear receptors. A T3-binding protein in rat kidney cytosol mediates T3-uptake by kidney mitochondria, perhaps by donating its T3 to a T3-receptor in the mitochondrial outer membrane, which is transported by other T3-carrier proteins in the intermembrane space, to a T3-receptor in the inner membrane. ${ }^{214}$

Transport of LT3 across the cytosol seems to be tissue-specific. The T3-pump in the plasma membrane or the cytosol is invoked as the source of a highly selective differential effect of a T3-analogue on heart and liver. To measure the contribution of the pump to the selectivity, Underwood et al. ${ }^{614}$ compare binding and displacement of LT3 and analogues in vivo and in vitro (to eliminate operation of the pump). As biologic responses to nuclear-receptor-binding, they assay mitochondrial glycerol-3-phosphate dehydrogenase and depression of plasma [cholesterol] in hypothyroid rats $48 \mathrm{hr}$ after one dose of LT3 or analogue. The 3'-pyridazinone-3,5-dibromothyronine (SK\&F-94901) is highly selective for liver: it has $18 \%$ of the inducing potency of LT3 in liver ( $0.1 \%$ in heart), liver binds it in vivo $50 \%$ as well as LT3 (heart, $1.3 \%$ ), and liver nuclei bind it in vitro $0.9 \%$ as well as LT3 (heart, 2\%). LT3 and SK\&F-94901 depress plasma [cholesterol] effectively and equally (see Section II.H). This selective access to liver nuclei is proposed to reside in the hormone-concentrating mechanisms, presumably in the energy-dependent plasma membrane and/or cytosol hormone-transporters - where hepatic cytosol may contain more SK\&F-94901 than heart cytosol either because hepatocytes accumulate it more actively or cardiomyocytes exclude it. DT4 has no selective potency, and induces glycerol-3-phosphate dehydrogenase in either heart or liver about $10 \%$ as effectively as LT3, as might be expected from the relative binding of DT4 and LT3 by the nuclear receptor.

\section{LIPID METABOLISM}

\section{A. Cell Lipid Composition}

Thyroid state influences cell and plasma lipid composition, but some reports are contradictory, probably because of differences in degree and duration of abnormal thyroid levels, and the many other effectors of lipid composition. Lipids in plasma of hyperthyroid rats, as compared with normals, show the expected decreases in free and esterified

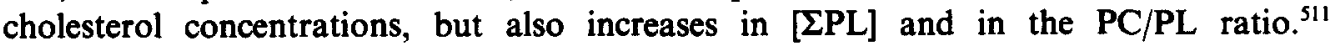
Erythrocyte membranes do not equilibrate lipid content with plasma, as is shown by their greater cholesterol content and normal PC/PL ratio, although PL/protein also increases; 
the PL contents of 18:1, 18:2 and 20:4 acyls remain unchanged. In livers of rats, either hypo- or hyperthyroidism halves total lipid content, while hypothyroidism depresses cholesterol/protein by $20 \%,{ }^{396}$ Ellefson and Mason ${ }^{130}$ find TG content low in hypothyroids, PL contents high in hyperthyroids; Tata ${ }^{596,598,599}$ finds diminished PL/protein ratios in livers of hyperthyroid rats.

In hepatic microsomes Faas and Carter ${ }^{140.141}$ report that hypo- or hyperthyroidism leaves unaltered the contents of PL, TG and cholesterol, and the distribution of the different PL classes. On the other hand, Ruggiero et al. ${ }^{510}$ see that hyperthyroidism decreases microsomal PL and cholesterol contents.

Hyperthyroidism induced with our original enormous doses of LT4, $40 \mu \mathrm{g} / \mathrm{g} / \mathrm{day}^{246}$ and given for 6 days, increases the PL content of rat liver mitochondria but does not change the proportions of the PL classes (although no CL fraction was distinguished), whilst hypothyroidism does not change either in the studies by Nelson and Cornatzer. ${ }^{424}$ In liver mitochondria of our hypothyroid rats, we find normal amounts of total lipids, PL and NL per $\mathrm{g}$ protein and increased CL/PL ratios. ${ }^{248}$ Studies combining measurements of CL and cytochrome $a$ contents per $\mathrm{g}$ protein in whole cell homogenates and in purified mitochondria, with stereological micrographic analyses of liver cells and mitochondria, have compared hypothyroids and normals, and used a prolonged T4-treatment (doses every other day for 21 days). ${ }^{277}$ Hypothyroidism selectively decreases, and T4 increases, the relative area of mitochondrial inner membranes, but the ratios of mitochondrial/liver protein and mitochondrial/cell volume do not change. In hypothyroid cells and mitochondria, CL and cytochrome $a$ contents per $\mathrm{g}$ protein decrease $20-40 \%$ below normal levels; T4, $0.1 \mu \mathrm{g} / \mathrm{g}$, restores normal values. When normal rats receive $1.2 \mu \mathrm{g} / \mathrm{g} \mathrm{T} 4, \mathrm{CL}$ and cytochrome $a$ contents increase to $20-40 \%$ above normal.

Mitochondrial membranes also proliferate in thyrotoxic rat hearts. ${ }^{460}$ Taken together with data showing that cell $\Sigma$ PL contents are $27 \%$ lower in hypothyroids than in euthyroids, ${ }^{596}$ and our data exhibiting high CL/PL ratios in hypothyroids, it would appear that hypothyroidism, or this prolonged T4-regimen, alters amounts of $\Sigma$ PL per $\mathrm{g}$ protein more severely than amounts of CL. Thyroid state affects the CL contents of individual liver mitochondria rather than the number of mitochondria per cell. Reasons to think that the $\mathrm{CL} / \mathrm{PL}$ ratio of such an altered mitochondrion regulates its inner membrane properties are discussed in Section IV.

Fatty acyl compositions of PL are more consistently found to be thyroid-dependent, although details vary. Some of the effects of thyroid state on PL fatty acyl composition result from changes in the fatty acyl proportions in one or more PL classes, others from a shift in the proportions of PL classes that have distinctive fatty acyl compositions. ${ }^{248}$ For example, CL contains much more 18:2 acyls and less other PUFA than PC, PE, or PI. ${ }^{153}$ Liver PL from hypothyroid rats, as compared with euthyroids, have slightly lowered 18:2 and $20: 4$ contents, and vigorous LT4 treatment $(2 \mu \mathrm{g} / \mathrm{g} /$ day $\times 14$ days $)$ of either hypothyroid or normal rats increases percentage contents of $20: 4$ while decreasing percentage contents of 18:2, indicating that T4 activates the 'degradation' of hepatic unsaturated fatty acyls. ${ }^{396,471}$ In other studies, ${ }^{130}$ liver phospholipids in hypothyroid rats have contents of 16:0,18:1 and 18:2 that are $80 \%$ above normal levels, and $20: 4$ contents that are $70 \%$ below normal, and what might be a 20:3 acyl appears; in thyrotoxic rats, 18:1 and 18:2 contents increase by $90 \%$, and $20: 4$ decreases $25 \%$. Since the hormone augments adipose tissue 16:0 and 18:1 contents, Ellefson and Mason ${ }^{130}$ propose that the hormone stimulates fatty acid de novo synthesis, fatty acid mobilization from fat $\rightarrow$ liver, and desaturations of saturated and unsaturated fatty acids. In hypothyroid hamsters, liver lipids are slightly depleted in 18:2; in thyrotoxic hamsters, $18: 2$ and $20: 4$ decrease more severely while 18:1 content increases. ${ }^{468}$ The results of Peifer ${ }^{471}$ on thyroid effects are more difficult to fit into the pattern because the rats were fed diets containing $1 \% 18: 2 \omega 6,1 \% 18: 3 \omega 3$, and $8 \%$ saturated fatty acids, and made hyperthyroid by inclusions of dessicated thyroid powder for 2 months. In these hyperthyroids, liver lipids were $40 \%$ below untreated 'controls' in percentage contents of $18: 2$ and $22: 6$, while in heart lipids, 18:2 percentage was halved and 22:6 percentage was 2.6-times elevated. This diet would produce EFA-deficiency as 
well as the ready replacement of cardiac $\omega 6$-PUFA by $\omega 3$-PUFA noted by Gudbjarnason et al.; ${ }^{196}$ the hormone was thought to accelerate the desaturative biosynthesis of both $22: 6$ and $20: 4$ from their dietary precursors.

Since cell PL are almost all in membranes, it is to be expected that organelle and cell membranes reflect the PL fatty acyl composition changes. Liver mitochondrial fatty acyl profiles in thyrotoxic rats have slightly lower $18: 2$ and $33 \%$ lower $20: 4$ percentages. ${ }^{478}$ Heart mitochondria from hypothyroid rats and hamsters show no fatty acyl changes, while those in thyrotoxics have slightly lowered $18: 2 ;{ }^{468,575}$ this is discussed further in Section IV. Table 1 compares total lipid contents of fatty acyl groups in liver mitochondria prepared from fasted control or hypothyroid rats, or from rats of either group killed after injection(s) of LT3. ${ }^{235,236}$ In our studies, hypothyroidism elevates mitochondrial contents of $18: 2,18: 3 \omega 6$, and $20: 3 \omega 6$ fatty acyls and depletes $20: 4$ acyls. One LT3-injection into hypothyroid rats rapidly substitutes saturated acyls for a part of the unsaturated fatty acyls. The hormone depresses mitochondrial 18:2, 20:4 and 22:6 fatty acyl contents to as low as $50 \%$ of the levels in untreated hypothyroids by $1-2.5 \mathrm{hr}$. The $35 \%$-increased contents of $16: 0$ and 18:0 acyls replace the polyenes, and the UI diminishes to $71 \%$ of that in untreated hypothyroids and to $62 \%$ of that in the euthyroid controls. Only weeks-long EFA-deficiency (see Section IV.E), or a few hours of feeding carbohydrate to previously starved euthyroid rats (see Section II.B), depletes liver mitochondrial $\omega 6$-PUFA to this extent. Three-day LT3-treatment of control rats also raises liver mitochondrial 18:0 content but, in addition, depletes 18:2 and 20:4 acyls.

Changes in oxidative phosphorylation at $25^{\circ} \mathrm{C}$ accompany the early depressions of mitochondrial PUFA contents within $3 \mathrm{hr}$ after hypothyroid rats are injected with LT3 (see Section IV.A), as well as during the more chronic depletions of w6-PUFA in EFAdeficiency (see Section IV.E), but do not appear to have been examined during carbohydrate refeeding. Altered thermotropic properties of partial reactions also occur in liver and heart mitochondria when thyroid status is changed (see Section IV). These findings indicate that the changes in mitochondrial fatty acyl composition are great enough to have functional consequences.

Liver microsomes from hypothyroid rats have abnormal fatty acyl compositions similar to those in their mitochondria, but these have no effect on $\Delta 9$-desaturase activity. ${ }^{243}$

TABLE 1. Fatty Acyl Compositions of Liver Mitochondria of Hypothyroid and Control Rats, and Changes after LT3 Injection(s)*

\begin{tabular}{|c|c|c|c|c|c|c|}
\hline \multirow{2}{*}{$\begin{array}{l}\text { Fatty } \\
\text { acyls }\end{array}$} & \multirow{2}{*}{$\begin{array}{l}\text { Controls } \\
(6)\end{array}$} & \multirow{2}{*}{$\begin{array}{l}\text { Hypothyroids } \\
\text { (9) }\end{array}$} & \multicolumn{3}{|c|}{$\begin{array}{l}\text { Hypothyroids + LT3 } \\
\text { (hours after injection) }\end{array}$} & \multirow{2}{*}{$\begin{array}{l}\text { Controls } \\
\text { + LT3 (4) }\end{array}$} \\
\hline & & & $0.5(2)$ & $1.0-2.5(8)$ & $4.0(3)$ & \\
\hline $16: 0$ & 12.6 & $16.0^{\mathrm{b}}$ & 16.2 & $21.2^{\mathrm{E}}$ & 21.1 & 16.7 \\
\hline $18: 0$ & 17.3 & 17.2 & 18.5 & $23.4^{\mathrm{D}}$ & 16.5 & $24.6^{\mathrm{E}}$ \\
\hline $18: 1$ & 17.9 & 18.2 & 14.3 & 19.6 & 21.7 & 15.1 \\
\hline $18: 2$ & 18.7 & $24.1^{b}$ & 22.9 & $17.6^{\mathrm{E}}$ & 18.8 & $12.5^{\mathrm{A}}$ \\
\hline $18: 3$ & 0.3 & $1.3^{\mathrm{c}}$ & 1.2 & 1.5 & 1.8 & 2.5 \\
\hline $20: 3$ & 1.0 & $2.0^{\mathrm{d}}$ & 2.9 & 1.4 & 1.8 & 0.7 \\
\hline $20: 4$ & 23.5 & $16.7^{b}$ & 16.5 & $9.4^{\mathrm{C}}$ & 9.6 & $16.9^{\mathrm{E}}$ \\
\hline $22: 6$ & 4.6 & 3.2 & 2.6 & $1.5^{\mathrm{B}}$ & 3.1 & 4.7 \\
\hline \multicolumn{7}{|l|}{ Unsat. } \\
\hline Index & 189 & 167 & 166 & $118^{B}$ & 136 & 162 \\
\hline$" \omega 6$ & 131 & 122 & 126 & $81^{D}$ & $84^{E}$ & 105 \\
\hline$" \omega 3$ & 29 & 26 & 21 & $17^{\mathrm{D}}$ & 22 & 36 \\
\hline
\end{tabular}

*Fatty acyl percentage contents are shown as means; for 55 values, the coefficient of variation is $16.6 \% \pm 1.9 \% \mathrm{SE}$. The number of experiments, each on one rat, is in parentheses. The unsaturation index (U.I. $=\Sigma[\%$ fatty acyl group $\times$ number of unsaturated bonds]) is shown for all unsaturated fatty acyl groups and separately for $\omega 6$ - and $\omega 3$-acyls. Some hypothyroid rats were injected with LT3 (+LT3) intraperitoneally, $1 \mu \mathrm{g} / \mathrm{g}$, and killed at the times indicated; some control rats were similarly injected with LT3, $1 \mu \mathrm{g} / \mathrm{g} /$ day for 3 days, and killed on the 4th day (controls $+\mathrm{LT} 3$ ). Student's $t$ test was used for group comparisons: $p<{ }^{\mathrm{aA}} 0.001 ;{ }^{\mathrm{b}} 0.005 ;{ }^{\circ} 0.01 ;{ }^{\mathrm{C} D} 0.025 ;{ }^{\mathrm{eE}} 0.05$; lower case symbols denote $p$ values for comparisons of Hypothyroids vs. Controls, upper case symbols for comparisons of Hypothyroids + LT3 vs. Hypothyroids and Controls + LT3 vs. Controls. 
LT3-injection changes microsomal composition slightly later and less extensively than in mitochondria. The proportion of $20: 4$ acyls falls at $2.5 \mathrm{hr}$ and reaches $75 \%$ of the hypothyroid level ( $49 \%$ of the control level) at $4.0 \mathrm{hr}$. With the accompanying decrease in $22: 6$ acyl percentage, the unsaturation index falls to $89 \%$ of the hypothyroid value at $4 \mathrm{hr}$. Three daily injections of LT3 into control rats have little effect on microsomal fatty acyl composition, other than to increase 18:0 content and decrease the $\omega 6$-unsaturation index.

In liver mitochondria of hypothyroid rats, it is the phosphatidylcholines and phosphatidylethanolamines that contain excess 18:2 fatty acyls and are depleted in 20:4 acyls, while the CLs have normal contents of $18: 2$ but the ratio CL/PL is increased. ${ }^{248}$ Hepatic nuclear ${ }^{549}$ phospholipids prepared from hypothyroid rats show the same abnormal fatty acid composition as mitochondria (Table 1) and microsomes, suggesting a general defect in unsaturated fatty acid metabolism. Because laboratory diets for rats contain no arachidonic acid, the desaturative biosynthesis of $20: 4$ fatty acyls from dietary $18: 2$ seems at fault. Although fatty acyl-CoA $\Delta 6$-desaturase activity is cited as 'rate-limiting' in this conversion, ${ }^{56}$ the appearance of $20: 3 \omega 6$ acyls suggests a more limiting defect in the $\Delta 5$-desaturase in hypothyroidism. Thyroid regulation of desaturase activities is discussed below (see Section II.I).

\section{B. Fatty Acid Synthesis}

Thyroid status is one of the regulators of de novo fatty acid synthesis. In general, rapid $(0-24 \mathrm{hr})$, reversible modifications of existing enzymes regulate fatty acid synthesis by several mechanisms. ${ }^{621}$ Allosteric effectors signal intracellular conditions, and cyclic nucleotide- or $\mathrm{Ca}^{2+}$-mediated phosphorylation/dephosphorylation regulates under hormonal control for whole-organism needs. The acetyl-CoA carboxylase, ${ }^{445}$ which is said to be 'rate-limiting' under most circumstances, is rapidly activated by citrate and inactivated by long-chain fatty acyl-CoA, but no certain thyroid influence via these effectors is established. Phosphorylation inactivates, and dephosphorylation reactivates, the acetylCoA carboxylase and fatty acid synthetase enzymes. An appropriate stimulus starts synthesis of some lipogenic enzymes even more quickly than the actions of rapid effectors or covalent modifiers. Synthesis rather than degradation is stated to regulate the activity of the fatty acid synthetase, ${ }^{5}$ the malic enzyme, ${ }^{177}$ the glucose-6-phosphate dehydrogenase, and the 6-phosphogluconate dehydrogenase, through the selective expression of genes and the nuclear synthesis or supression of mRNAs (see Section II.B.2).

Hypothyroidism in developing or adult rats depresses hepatic fatty acid synthetase activity by $50 \%$, and diminishes but does not abolish carbohydrate-induced activation of fatty acid synthetase. ${ }^{336,620}$ Cultures of hepatocytes obtained from hypothyroid rats synthesize fatty acids from acetate half as fast as normals. ${ }^{173}$ Incubation of these hepatocytes with $\sim 1 \mathrm{nM}$ LT3 stimulates synthesis in $2 \mathrm{hr}$ and triples the rate by $4 \mathrm{hr}$ independently of protein synthesis, since cycloheximide fails to inhibit. Greater LT3 concentrations accelerate fatty acid synthesis in hepatocytes from normal rats $37 \%$ in $4 \mathrm{hr}$. LT4-injection of normal rats doubles the amount of hepatic citrate lyase and triples that of malic enzyme in $7.5 \mathrm{hr},{ }^{170}$ and increases fatty acid synthetase and acetyl-CoA carboxylase activities more slowly. ${ }^{366}$

Starvation, ${ }^{7,8,170,648}$ diabetes, ${ }^{105}$ or hypophysectomy ${ }^{324 a}$ decrease hepatic fatty acid synthesis more severely (to $<10 \%$ of normal), as well as activities of fatty acid synthetase, acetyl-CoA carboxylase, malic enzyme, citrate lyase, and glucose-6-phosphate dehydrogenase. In starved or diabetic rats, the liver contains considerable amounts of immunoreactive fatty acid synthetase that is enzymatically inactive. Carbohydrate fed to fasted rats ${ }^{648}$ increases fatty acid synthetase in two phases. Within $1 \mathrm{hr}$, additional inactive fatty acid synthetase is synthesized. After $3 \mathrm{hr}$, fatty acid synthetase activity begins to increase, in parallel with incorporation of labeled 4'-phosphopantotheine into fatty acid synthetase apoenzyme, forming holoenzyme. We do not know what makes existing apoenzyme take up its prosthetic group. Refeeding accelerates synthesis of malic enzyme 
and citrate lyase in $6 \mathrm{hr} .{ }^{170}$ When rats are refed with carbohydrates for 15 days, depressed activities of hepatocyte cytosol acetyl-CoA carboxylase, fatty acid synthetase, malic enzyme, citrate lyase, and glucose-6-phosphate dehydrogenase coordinately rise to levels many times normal. ${ }^{170}$ This coordination prevents the increased flux of metabolites from accumulating behind each relatively slowed step. Supplementation of fat-free diet with $18: 2$ or $20: 4$ (but not $16: 0$ or 18:1) dampens the induction of all these enzymes; as discussed under Section II.I, major regulatory properties are also ascribed to dietary linoleate through its potent inhibition of liver fatty acid synthetase and the fatty acyl-CoA $\Delta 9$-desaturase. ${ }^{279}$ Thyroid state controls the hepatic uptake and esterification of administered 18:2 266 , thereby further regulating lipogenic enzymes. The suppressive effect of fat-feeding on the carbohydrate-inductions of acetyl-CoA carboxylase, fatty acid synthetase, and malic enzyme diminishes with aging of rats, and is relieved by T3treatment, more in young rats than in old. ${ }^{163}$ Thyroid hormones and refeeding fasted rats with carbohydrates exert synergistic effects on lipogenic enzyme gene expression (see Section II.B.2).

Effects of one $\mathrm{T} 3$ injection on hepatic fatty acid synthetase and acetyl-CoA carboxylase have been measured in hypophysectomized ${ }^{324 a}$ or diabetic ${ }^{105}$ rats. T3 given to hypophysectomized rats does not increase fatty acid synthetase activity from 4-12 hr later, but Kumar et al. ${ }^{324 a}$ did not measure short-term effects on fatty acid synthetase amount or synthesis. Long-term, T3 activates fatty acid synthetase and acetyl-CoA carboxylase only after $24 \mathrm{hr}$, to reach maximal activity at 3-4 days; injecting T3 twice over 7 days accelerates activities of fatty acid synthetase and acetyl-CoA carboxylase 15- to 20-fold, and synthesis of fatty acid synthetase $\sim 10$-fold. A different biphasic pattern is seen after diabetic rats are injected with T3. ${ }^{105}$ In 4-12 hr, activities of fatty acid synthetase and acetyl-CoA carboxylase rise slowly, together with conversion of apoenzyme $\rightarrow$ holoenzyme and accumulation of immnoreactive fatty acid synthetase; however, cycloheximide or actinomycin $\mathrm{D}$ does not block the increase in activities or the parallel holoenzyme formation. In a second stage from $24-72 \mathrm{hr}$ after T3 injection, a more rapid increase in activities and fatty acid synthetase antibody titer occurs that is inhibited by blockage of transcription or translation and so represents net protein synthesis. Comparable long-term T3 treatment of diabetic animals stimulates hepatic fatty acid synthesis from all precursors. ${ }^{584 a}$ Here, insulin does not mediate $\mathrm{T} 3$ effects. Insulin given to diabetic rats acts more quickly than T3, and increases fatty acid synthetase activity 5-fold in $6 \mathrm{hr}, 20$-fold in $12 \mathrm{hr}$ (see Kumar et al. ${ }^{324 a}$ for Refs). In contrast, in avian liver explant culture, insulin induces a 5 -fold increase in fatty acid synthetase; T3 or hydrocortisone potentiate the induction, individually 2-fold, together 4-fold; without insulin, they are ineffective. ${ }^{150.285}$ Apparently, these cultures require insulin, and T3 or hydrocortisone play supportive roles in induction of fatty acid synthetase.

\section{Protein Phosphorylation-Dephosphorylation}

As noted above, cAMP-dependent and -independent protein kinases inhibit ratelimiting enzymes of fatty acid synthesis. In vitro, sequential additions of protein kinases and phosphatases interconvert active and inactive forms of an enzyme. ${ }^{264,444}$ For example, in vitro dephosphorylation activates pigeon liver fatty acid synthetase 15-fold, and phosphorylation inactivates to the same degree. ${ }^{487}$ Usual procedures for fractionation indicate that under normal physiologic conditions $90 \%$ of a lipogenic liver enzyme, $\beta$-hydroxy- $\beta$-methylglutaryl-CoA reductase (HMGR, see Section II.H), exists in an inactive phosphorylated form. ${ }^{649}$ In this case, decapitating conscious rats is shown to result in almost complete phosphorylation of enzyme in seconds. Using anesthetized fed rats, and cold-clamped liver to eliminate the delay during centrifugal fractionations, HMGR is $80 \%$ active. Such procedures may well be critical in correlating the receptor-mediated thyroid-state effects on other lipogenic enzymes as well.

Thyroid treatment induces several protein kinases and increases the in vivo phosphorylation of endogenous proteins. In the absence of valid examinations of phos- 
phorylation states of lipogenic enzymes, evidence for direct connections with thyroid levels remains circumstantial-we discern motive, means, and opportunity but have no witness to dephosphorylation. In the myocardium, $2 \mathrm{hr}$ after T3-injection, the kinase activity of nuclear non-histone proteins increases for each substrate tested (histones, casein, phosvitin). ${ }^{171}$ Activity doubles after 3 daily T3-injections but even continued treatment for more than 7 days does not prevent the return of kinase activities to control levels. T3-treatment also activates two isozymes of a myocardial cytosol cAMP-dependent protein kinase $I$ in $2 \mathrm{hr}$; detectable rises in [cAMP] and [cGMP] start at $4 \mathrm{hr} .{ }^{48}$ The significance of these early kinase activations or inductions for lipogenesis is uncertain, since the heart has little lipogenic enzyme apparatus.

In rat liver, T3-treatment produces stimulations analogous to those in heart, but more slowly. When hepatocytes from fetal rats are incubated with T3 for 3 days (but not for $5 \mathrm{hr}$ ) increased activity of nuclear protein kinases and susceptibility of chromosomal non-histone proteins to phosphorylation are observed. ${ }^{199}$ Nuclear non-histone proteins extracted from livers of rats after 2 daily injections of $\mathrm{T} 3$ are increased in phosphorylative actions on casein and phosvitin by $60-90 \%$; partially purified fractions of nuclear extracts obtained after 8 daily injections are 4 times more active than those from control rats. ${ }^{324,501}$ These T3-induced fractions, as compared with controls, have different $\mathrm{pI}$ values, are more heat-labile, are less sensitive to inhibition by a sulfhydryl reagent, and have greater autophosphorylative activity. ${ }^{324}$ Thus T3 seems to be evoking gene expression of 'new' protein kinases. Since these kinases, like those in heart, disappear even with continued T3-treatment, a down-regulation may decrease the number of nuclear receptors ${ }^{521}$ or LT3 access to the receptors.

In livers of hypothyroid rats, phosphorus-content of ribosomal proteins, and activities and cAMP-binding of soluble protein-kinases, are below euthyroid levels. ${ }^{100}$ Nuclear chromatin non-histone proteins autophosphorylate at a subnormal level, except for one large fraction that is much more active than normal. ${ }^{324}$ Hypothyroidism decreases both nuclear and cytosolic casein kinase activity and T3-injection restores these activities in $15 \mathrm{hr}$. Protamine kinase in the cytosol is more than normally active, and decreases after T3-treatment. ${ }^{501}$ Thus, thyroid state appears to modulate specific protein kinases, and hypothyroidism even activates some nuclear kinase(s) while inactivating others. Further characterization of thyroid-responsive cytosolic protein kinases in livers of hypothyroid rats $^{416,469,470}$ shows that the cAMP-independent histone kinases are normally active while the casein kinases have half-normal activity. T4-treatment restores one fraction of the cAMP-dependent casein kinases to normal levels in $2-5$ days, ${ }^{469,470}$ and these are responsible for the phosphorylation of the cytosolic proteins. ${ }^{416}$ In addition, T3-administration to hypothyroid rats for 3 days activates a cytosolic cAMP-dependent protamine kinase. ${ }^{416}$ Lag periods of days must be compared with the few hours it takes for T3 to activate hepatic lipogenic enzymes that are well documented to be activated when dephosphorylated. Thus, in vivo studies on protein phosphorylation in hypothyroid rats fasted-and-refed-glucose and injected with ${ }^{32} \mathrm{P}_{\mathrm{i}}$ and $\mathrm{T} 3$ before killing, ${ }^{93}$ are instructive because fasted/refed animals normally dephosphorylate hepatic cytosolic lipogenic enzymes. Before the hypothyroid rats receive T3, nuclear proteins incorporate injected ${ }^{32} \mathrm{P}$ at $15-35 \%$ below normal levels-so hypothyroidism suppresses nuclear protein phosphorylation. T3-injection progressively increases ${ }^{32} \mathrm{P}$-incorporation to reach significant levels $6 \mathrm{hr}$ later. Cytosol protein phosphorylation increases even more slowly. Again, the lag time in the nucleus seems too great to account for the $40 \mathrm{~min}$ that it takes T3 to activate the nuclear DNA-dependent RNA polymerase II (Ref. 286; but see Ref. 608). This is curious because both the hepatic polymerases I and II are activated by either cAMP-dependent or -independent protein kinases and inactivated by phosphoprotein phosphatases. ${ }^{319}$

Phosphoprotein phosphatases are of particular interest when one regards the number of enzyme reactions in the pathways of lipid metabolism (and of carbohydrate metabolism, for that matter) that are activated both by T3-treatment and by protein dephosphorylation. ${ }^{211,212,267,319}$ No protein phosphatase has as yet been connected with thyroid 
state. However, some of these phosphatases are indirectly activated through the phosphorylation of phosphatase-inhibitor proteins by either cAMP-dependent or -independent protein kinases, ${ }^{265}$ and thyroid state regulates activity of protein kinases of either persuasion, as well as [cAMP] (see Section III.B).

Covalent enzyme modifications by phosphorylation/dephosphorylation mechanisms ${ }^{211,212}$ may also account for some hormonal and dietary interactions with T3 effects. A preliminary note reports that starvation (which was taken as a glucagon signal) decreases the degree of phosphorylation of several histones and non-histones in isolated nuclei incubated with $\left[\gamma{ }^{32} \mathrm{P}\right] \mathrm{ATP}$, and refeeding carbohydrate (taken as an insulin signal) rephosphorylates nuclear non-histones. ${ }^{344}$ In contrast, insulin promotes dephosphorylation in hepatic cytoplasm. Dephosphorylation appears to regulate important cytosol enzymes in the pathways that convert glucose-6-phosphate to cytoplasmic acetyl-CoA, viz. phosphofructokinase 2, pyruvate kinase (a cAMP-dependent protein kinase inactivates), and pyruvate dehydrogenase. Citrate lyase is subject to reversible phosphorylation, but its activity remains unaltered. As is discussed elsewhere in this review, conversion of acetyl-CoA to lipids also involves enzymes activated by dephosphorylation, ${ }^{319}$ insulin, or thyroid hormone; these include acetyl-CoA carboxylase, glycerol-3-phosphate acyltransferase (inactivated by a cAMP-kinase), HMG-CoA reductase, cholesterol- $7 \alpha$-hydroxylase, cholesterol ester-hydrolase (activated by a cAMP-kinase), and possibly fatty acyl-CoA $\Delta 9$-desaturase.

\section{Gene Expression}

As noted in the preceding section, $\mathrm{T} 3$ or $\mathrm{T} 4$ increase amounts and synthetic rates of several enzymes involved in fatty acid synthesis, in some cases after activating inactive enzyme. The rapid progress of studies on the T3-induction of such enzymes, and its relationship to the mechanisms whereby dietary carbohydrate induces lipogenic enzyme activities in the livers of starved animals, ${ }^{604}$ has been updated, ${ }^{381,451-454}$ the details of information transfer are beyond the scope of this review. The enzymes most studied are fatty acid synthetase, acetyl-CoA carboxylase, citrate lyase, malic enzyme, glucose-6phosphate dehydrogenase, and 6-phosphogluconate dehydrogenase. Additional rapidly induced lipogenic enzymes are the mitochondrial glycerol-3-phosphate dehydrogenase (see Section II.E) and the fatty acyl-CoA $\Delta 9$-desaturase (see Section II.I). The mechanisms of the synergism between carbohydrate-feeding and T3 treatment do not involve a carbohydrate-induced increase in nuclear T3-uptake per dosage, or the number and affinity of the T3-receptor sites. Further indication that insulin is not necessary for the T3-effect lies in observations that fructose-feeding restores the depressed T3-induction of hepatic malic enzyme in diabetic rats, since fructose is metabolized in the absence of insulin (see Kumar et al. ${ }^{324 a}$ for Refs). It is therefore postulated that some intracellular product of glucose metabolism induces malic enzyme, and that T3 acts as a constant multiplier of that signal in the nucleus. The nature of this metabolite is not known, but it appears to be of mitochondrial origin, since dichloroacetate promotes its action: dichloroacetate activates the pyruvate dehydrogenase by inhibiting the dehydrogenase kinase in the dehydrogenase complex.

When injected in vivo, the hormone augments or suppresses 21 out of 250 separated liver mRNAs. A T3-induced increase in pituitary growth hormone level effects the changes in 7 of these 21 mRNAs. Carbohydrate-feeding also changes 8 of these 21 , and in the same direction as T3. A dose of T3 large enough to convert receptor occupancy from the hypothyroid to the hyperthyroid level augments 4 of the mRNAs in carbohydrate-fed animals, from 4-fold to 13 -fold. ${ }^{376}$ Three of them are mostly induced in the range of T3-occupancy from euthyroid to hyperthyroid, as is malic enzyme-mRNA, mRNA (see below), and activity of the lipogenic enzymes. Only $1 \mathrm{mRNA}$ is induced at $4 \mathrm{hr}$ (the earliest time of examination), the other 3 at $>8 \mathrm{hr}$. Obviously, only a few more proteins are involved in these rapid inductions than the half-dozen or so lipogenic enzymes studied, but not nearly enough proteins to account for the great diversity of thyroid effects. 
And the $11 \mathrm{hr}$ of $\mathrm{T} 3$ residence in the liver ${ }^{537}$ is too short to account for additional T3-receptor-mediated, long-term syntheses.

Several liver mRNAs respond very rapidly to T3. T3 and carbohydrate-feeding each start hepatic malic enzyme synthesis in about $2 \mathrm{hr}$, so neither works by stimulating the other. The amount of malic enzyme-mRNA translated correlates with the increase in malic enzyme synthesis, so synthesis and/or processing of the mRNA are primary events. These times are still longer than the $20-60 \mathrm{~min}$ after $\mathrm{T} 3$ injection reported for generation of a hepatic mRNA dubbed mRNA $_{\mathbf{S 1 4}}$. A heteronuclear precursor RNA for mRNA $_{\mathbf{S 1 4}}$ is said to appear as early as $10 \mathrm{~min}$ after a dose of $\mathrm{T} 3$ that saturates liver T3-receptors. ${ }^{420}$ The proportion of basal, unstimulated [mRNA $\mathrm{m}_{\mathrm{S14}}$ ] to total mRNA contents is high in lipogenic tissues: 10 times greater in adipose cells than in livers of males, and 3 times more in livers of females ( 7 times if they are lactating) than in males. Hypothyroidism drops the mRNA $\mathrm{S}_{\mathrm{S} 14}$ content in fat by $80 \% .{ }^{287} \mathrm{~T} 3$ raises $\left[\mathrm{mRNA}_{\mathrm{S} 14}\right.$ ] in lipogenic cells, to a varying degree. At $24 \mathrm{hr}$, a receptor-saturating dose of $\mathrm{T} 3$ increases mRNA $_{\mathrm{S} 14}$ in fat cells twice as much as in liver cells. ${ }^{287}$

Non-lipogenic tissues have much less $\mathrm{mRNA}_{\mathrm{S1} 4}$ : brain contains about $6 \%$ as much as male liver, kidney, heart and spleen $<1 \%$; $\mathrm{mRNA}_{\mathrm{SI} \mid}$ in these tissues does not increase after $\mathrm{T} 3$ administration. Thus, the presence of $\mathrm{T} 3$ receptors in kidney and heart is not sufficient for $\mathrm{T} 3$ to evoke this mRNA or its product. The translation product of $\mathrm{mRNA}_{\mathbf{S 1 4}}$, a cytoplasmic polypeptide of $17 \mathrm{kDa}$, does not appear to be a component of enzymes or transporters involved in fatty acid metabolism that have registered sequences. From its temporal primacy after T3 treatment, from the involvement of the T3-induced carbohydrate signal with protein phosphorylation, from the early T3-activation of existing inactive lipogenic enzymes, and from the known T3-induction of nuclear protein kinases, ${ }^{93}$ one can not resist the speculation that this is a subunit of such a kinase (or a phosphoprotein phosphatase). This, despite the fact that so far T3 induces known kinases much too slowly, and has no demonstrated effects on phosphoprotein phosphatases.

Adipocyte nuclear receptors bind $0.4 \mathrm{ng} \mathrm{LT} 3 / \mathrm{mg} \mathrm{DNA}^{71}$ as compared with $0.6 \mathrm{ng} / \mathrm{mg}$ DNA in liver nuclei. However, there is more disagreement as to the effects of thyroid state on enzymes of fatty acid synthesis and $\mathrm{NADP}^{+}$reduction in adipose tissues. In hyperthyroid rats, Roncari and Murthy ${ }^{499}$ find a $40 \%$ decrease in the in vivo synthesis of fatty acids, activities of fatty acid synthetase and acetyl-CoA carboxylase, and total fatty acid synthetase; corresponding values in liver are $+40 \%$ to $+90 \%$. Contrarily, apparently equivalent LT4 or LT3 dose/time schedules increase fatty acid synthetase activity by $50-70 \%$ in white fat ${ }^{115,380}$ and rat neonate brown fat. ${ }^{205}$ But Diamant et al. ${ }^{115}$ find hyperthyroidism does not increase malic enzyme activity while it augments glucose-6phosphate dehydrogenase and 6-phosphogluconate dehydrogenase activities, whereas Mariash et al ${ }^{380}$ see a rise in malic enzyme activity and no changes in these dehydrogenases. In adipose tissue from hypothyroid rats, activities of fatty acid synthetase, malic enzyme, glucose-6-phosphate dehydrogenase, and 6-phosphogluconate dehydrogenase are reported to be depressed $40 \%$ to $80 \%$ below normal levels. ${ }^{380}$ In apparently similar adipose tissue, acetyl-CoA carboxylase and glucose-6-phosphate dehydrogenase $V$ values are $\sim 2.5$ times normal, and the specific activities of fatty acid synthetase and 6-phosphogluconate dehydrogenase are $50 \%$ to $80 \%$ above normal levels; in the report of Correze et al. ${ }^{97}$ T3-injection decreases glucose-6-phosphate dehydrogenase activity to near normal levels in $2-5 \mathrm{hr}$. This rapid inactivation is not attributable to any known activation of specific proteolysis, and the glucose-6-phosphate dehydrogenase is reported to be not subject to phosphorylation by cAMP-dependent protein kinases. ${ }^{267}$ Since responses of lipogenic enzymes expected from adipocyte LT3-receptor occupancy and subsequent gene expression are not observed by all investigators, it would appear that other regulatory mechanisms intervene, perhaps including altered states of phosphorylation.

The situation is somewhat cleared by studies of cultured precursors of adipocytes, in which effects of several hormones on lipogenic enzymes can be distinguished (see Ref. 634a). Thyroid hormones, insulin, growth hormone, or glucocorticoids induce adipogenic activities in clonal lines obtained from fibroblast-like cells. As measured by lipid 
synthesis and glycerol-3-phosphate dehydrogenase activity, T3 and T4 have no effects on primary cultures derived from adipose tissues, but insulin and glucocorticoids are required for conversion to adipocytes. T3 is essential for adipose differentiation of $o b 17$ cells ${ }^{169}$ and is active in preadipocytes kept in serum-free medium, but has no effect on rat preadipocytes under the culture conditions of Wiederer and Löffler. ${ }^{634 a}$ Incubation of $o b 17$ cells with $1.5 \mathrm{nM}$ T3 for 12 days first activates existing fatty acid synthetase, later increases the amount of fatty acid synthetase, and subsequently leads to accumulation of inactive enzyme that is still immunoreactive. ${ }^{169}$

Triiodothyronine induces lipogenic enzymes interactively with a number of hormones, including estrogens and luteinizing hormone (see discussion above, of the high basal [mRNA $\left.\mathrm{m}_{\mathbf{S 1 4}}\right]$ in females), growth hormone, glucocorticoids, and insulin. Insulin interactions are the most extensively studied. In all lines of adipocyte precursor cells, insulin induces a coordinate increase of acetyl-CoA carboxylase, fatty acid synthetase, malic enzyme, and citrate lyase. Insulin incubation with extracts of adipocyte plasma membrane normally promotes a kinase-mediated phosphorylation of Tyr in the insulin $\beta$-subunit receptor; in extracts from hypothyroid rats, this autophosphorylation response to insulin increases 2 to 3-fold, and T3-treatment of these hypothyroid rats depresses the insulin action to normal levels in $24 \mathrm{hr} .{ }^{99}$ Such a mechanism would seem to mediate an insulin-T3 antagonism, while covalent modifications might mediate collaboration.

One may ask what physiological purpose is served when calorigenic and anabolic hormones like T3 and T4 act so immediately on nuclear receptors to express genes for just a few lipogenic enzymes that mediate fatty acid and cholesterol (see Section II.H) synthesis, $\mathrm{NADP}^{+}$reduction, and perhaps fatty acyl-CoA desaturation (see Section II.I).

Elongation of palmitoyl-CoA and general fatty acid chain elongation by malonyl-CoA in liver microsomes accelerates up to 3-fold in rats pretreated with thyroid hormones for 21 days, and slows by $30-40 \%$ in rats made hypothyroid. ${ }^{300,336}$ The competing malonylCoA decarboxylase remains unchanged by LT3-treatment, and decreases slightly in hypothyroidism. Fatty acyl chain elongation by acetyl-CoA condensation in mitochondria is not affected by thyroid state, ${ }^{336}$ despite the thyroid-responsiveness of the carnitine palmitoyltransferase in the outer face of the inner membrane (see Section II.J), which is thought to intromit acyl-CoA for elongation. ${ }^{41}$

Brains of adult animals readily incorporate labeled acetate from the blood and synthesize fatty acids that appear in PL, but the slow turnover contrasts with that of other tissues. The acetyl-CoA carboxylase and the fatty acid synthetase of brain are similar to those of other tissues, except that they have longer biologic half-lives, and do not respond to dietary changes. ${ }^{644}$ Hypothyroidism, when induced from birth, depresses fatty acid synthetase activity in brain as well as in liver; fatty acid chain elongation in brain microsomes and mitochondria is also reduced. ${ }^{193 a}$ Daily T3 treatment of normal rats starting at 1 day of age increases brain microsomal chain elongation by malonyl-CoA 2.5-fold by day 6 , to synthesize those saturated, long-chain fatty acids characteristic of myelin lipids. Thyroid treatment of normal adult animals does not affect brain fatty acid synthetase activity in doses that stimulate the liver synthetase. ${ }^{620}$

\section{NADPH Generation and Oxidation}

\section{Cytoplasm: $N A D P^{+} \rightarrow N A D P H$}

Thyroid inductions of hepatic cytosol enzymes that reduce NADP ${ }^{+}$to NADPH include malic enzyme, glucose-6-phosphate dehydrogenase, and 6-phosphogluconate dehydrogenase, which are discussed in Section II.B.2. Fasting/refeeding does not induce the last two enzymes in hypophysectomized rats; partial return of adaptation to refeeding occurs after 10-14 days of treatment with T3 (25\%), hydrocortisone, or growth hormone; hydrocortisone + T3 (or growth hormone) restores induction $50 \%$, and all three produce a supernormal response. ${ }^{602,603}$ 


\section{Mitochondria: $N A D P^{+}+N A D H \rightarrow N A D P H+N A D^{+}$}

Mitochondrial energy-dependent pyridine nucleotide transhydrogenase is thought to play a role in lipid syntheses by mediating the transfer of reducing equivalents from mitochondrial NADH to NADPH, whence the citrate shuttle transfers the equivalents to the cytoplasm to regenerate NADPH-a role analogous to other thyroid-dependent cytoplasmic generators of NADPH (see above). The transhydrogenase also provides mitochondrial NADPH required in the oxidation of unsaturated fatty acids, ${ }^{35}$ and so contributes to $\beta$-oxidation. Among the auxiliary enzymes that metabolize the unusual $\Delta 3$-cis-enoyl-CoA or $\Delta 2$-trans- $\Delta 4$-cis-dienoyl-CoA esters to intermediates accessible to the normal $\beta$-oxidation enzymes are a 2-enoyl-CoA reductase and a 2,4-dienoyl-CoA 4-reductase, both of which use NADPH to reduce double bonds.

The hypothyroid state increases energy-dependent transhydrogenase activity but not energy-independent activity. ${ }^{136,137}$ In intact liver mitochondria, accelerated reduction of $\mathrm{NADP}^{+}$during the State $3 \rightarrow$ State 4 transition provides an indirect measurement. ${ }^{232,234}$ In submitochondrial particles where the enzyme is on the outer surface, manipulation of substrate concentrations provides measurements of kinetics. ${ }^{136,137}$ There the $V$ of the ATP-supported and the succinate-supported activities is doubled in hypothyroid preparations, while the $K_{\mathrm{m}}$ values remain unchanged. Since the kinetics of the energy-independent mode of the enzyme are unchanged, the amount of enzyme would not appear to have increased. Because these particles oxidize succinate at normal rates, the coupling between energy source and transhydrogenation is thought to be increased in hypothyroidism. Within 3 days after injecting hypothyroid rats with LT4 or LT3, energydependent activities decreased to the lower, normal levels. Possible mechanisms that involve membrane lipids in the selective thyroid-dependency of the energy-linked transhydrogenase are discussed in Section IV.B.9.

\section{Microsomes: $N A D P H \rightarrow N A D P^{+}$}

Hyperthyroidism increases, and hypothyroidism decreases, activities of flavoprotein components of hepatic microsomal NADPH-oxidizing systems (NADPH-cytochrome $c$ reductase and NADPH diaphorase). ${ }^{298,588}$ A small dose of LT3, given to hypothyroid rats, corrects activities within $20 \mathrm{hr} .{ }^{588}$ Hypothyroidism concomitantly increases amounts of component hemoproteins (cytochrome $b_{s}$ and P450). Some of these NADPH-dependent systems are involved in steroid metabolism (see Section II.H.1). Flavoprotein components of NADH-cytochrome $c$ reductase and NADH diaphorase are said to increase in activity, but we noted no change in NADH-cytochrome $c$ reductase activity in our hypothyroid rats. $^{243}$

\section{Fatty Acid Activation}

Long-chain fatty acyl-CoA synthetases (A in Fig. 1) in liver exist mostly in endoplasmic reticulum and in outer membrane of mitochondria, and much less in peroxisomes. Microsomal and mitochondrial enzymes are not identical. By their location and perhaps by their specificity, they segregate fatty acyls into a pool for glycerolipid synthesis (and/or chain elongation and desaturation) and a pool for oxidation, as has been shown for the two different acyl-CoA synthetases of the yeast Candida lipolytica. ${ }^{445}$ Injected labeled 18:2 is conserved from oxidation in vivo, ${ }^{95,394}$ although isolated heart mitochondria oxidize 18:2 faster than 16:0.57 Preferential esterification of 18:2 with $\mathrm{CoA}$ by the microsomal synthetase would direct 18:2 into glycerolipids or further desaturation, and away from mitochondrial oxidation. Triglyceride and PL synthesis in hepatocytes have different specificities for fatty acids. ${ }^{75}$ Triglycerides and diacylglycerides incorporate all fatty acids indiscriminately, in a pattern that reflects the composition of the suspending medium. Phospholipids, especially PE and PI, incorporate more 20:4 than do triglycerides when the medium contains $18: 2$ and $20: 4$.

Hepatic esterification of free fatty acids by long-chain acyl-CoA synthetase (A in 
Fig. 1) is reduced in thyrotoxicosis and increased in hypothyroidism, ${ }^{55,333,411}$ effects opposed to the rates of triglyceride lipolysis. This seems consistent with the absence of thermogenic cycling of triglyceride/fatty acid re-esterification in adipose tissue from T3-treated mice. ${ }^{63,64}$ Thyroid treatments do not alter the activity of the palmitoyl-CoA hydrolase (B, Fig. 1) of rabbit heart, ${ }^{295}$ and so hydrolysis does not appear to compete with esterification. Through thyroid stimulation of triglyceride lipolysis (see Section II.F), free fatty acids become more available for acyl-CoA synthesis as thyroid level increases. Thyroid state also controls CoA availability, but inconsistent effects are reported. Thus, low CoA contents of livers of thyroidectomized rats (other tissues are less depleted) increase to above normal levels with T4-treatment, ${ }^{591}$ while others report CoA depletion in livers of hyperthyroid rats, pigeons, and humans (see Ref. 226), and Müller et al. ${ }^{411}$ find increased CoA in perfused livers of either hyper- or hypothyroid rats. In rats made deficient in pantothenic acid, injection of T4 with pantothenate raises liver CoA content much more than does the vitamin alone ${ }^{611}$ suggesting thyroid effects on the enzymes and/or the 5 P-bonds involved in $\mathrm{CoA}$ synthesis. Compartmentation of $\mathrm{CoA}$ accounts for some of these discrepant reports. Liver mitochondrial CoA content per unit protein is normally 2-3 times cytosol CoA content; cytosol CoA decreases by $-40 \%$ below normal in hypothyroidism, and increases $35 \%$ above normal in hyperthyroidism, while mitochondrial CoA hardly changes. ${ }^{366}$ With the hypothyroid liver containing about $20 \%$ of cytosol CoA as malonylCoA, little free CoA must be available.

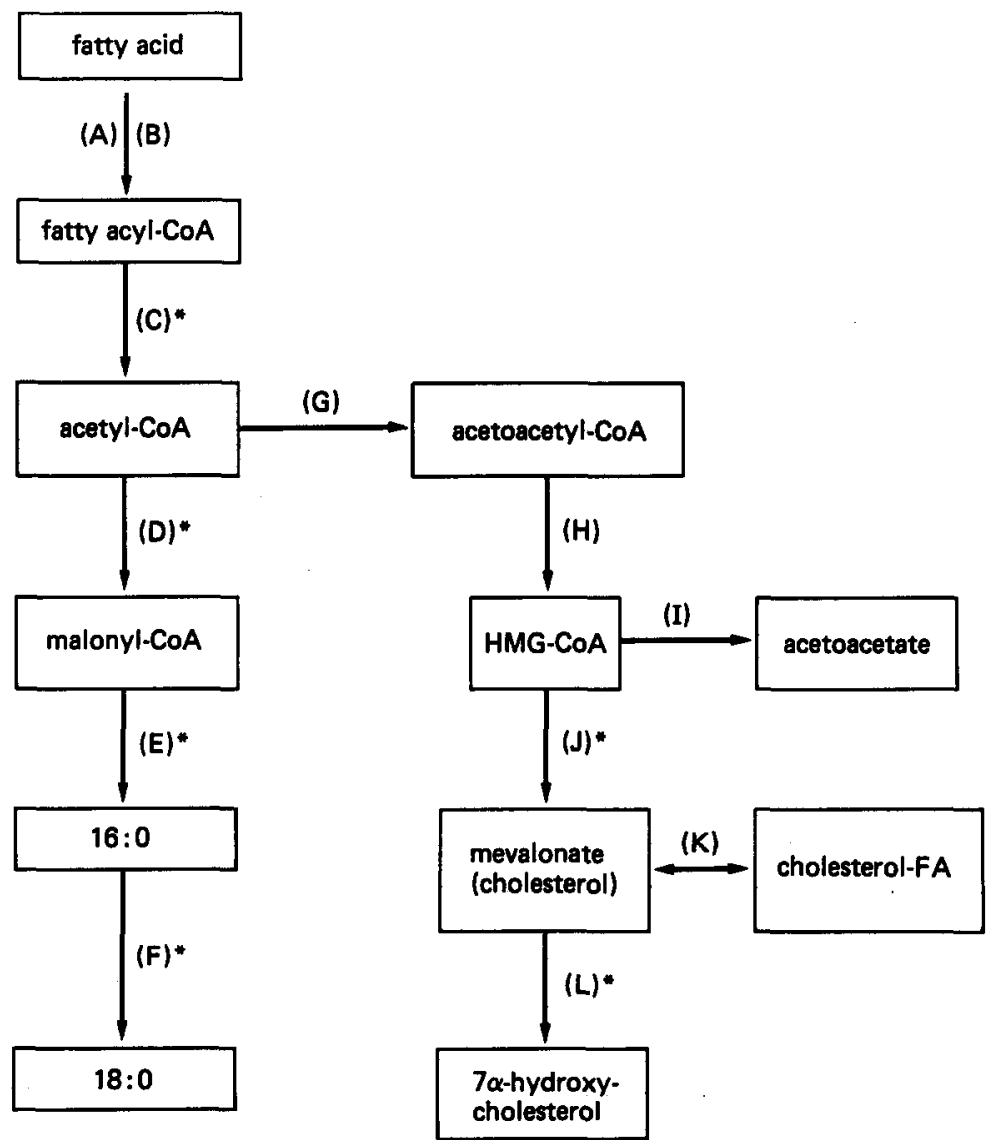

FIG. 1. Fatty acid and cholesterol metabolism: fatty acid $\beta$-oxidation, synthesis of 18:0, acetoacetate and $7 \alpha$-hydroxycholesterol; effects of thyroid state. ${ }^{*}$ Affected by thyroid state. (A) Fatty acyl-CoA synthetase; + CoA. (B) Fatty acyl-CoA hydrolase; -CoA. (C) $\beta$-oxidation. (D) Acetyl-CoA carboxylase; $+\mathrm{CO}_{2}$. (E) Fatty acid synthetase; $-\mathrm{CO}_{2}$. (F) Fatty acid elongation; +NAD(P)H. (G) Thiolase; + acetyl-CoA, - CoA. (H) HMG-CoA synthase; + acetyl-CoA, -CoA. (1) HMG-CoA lyase; + acetyl-CoA. (J) HMG-CoA reductase; + 2NADPH, -2NADP+, - CoA; subsequent synthesis of cholesterol. (K) Acyl-CoA: cholesterol acyltransferase, + acylCoA; cholesterol esterase, -FA. (L) Cholesterol $7 \alpha$-hydroxylase; +NADPH, $+\mathrm{O}_{2},-\mathrm{NADP}+$. 
TABLE 2. Distribution of the $\left[1-{ }^{14} \mathrm{C}\right]$ of Labeled Linoleate into Rat Liver Lipids 6 Hours after Injection ${ }^{248}$

\begin{tabular}{|c|c|c|}
\hline & Normals & Hypothyroids \\
\hline$\%$ of ${ }^{14} \mathrm{C}$ dose in $\Sigma \mathrm{L}$ & 3.5 & 0.9 \\
\hline$\%$ of $\Sigma L{ }^{14} \mathrm{C}$ in PL & 63.1 & 75.5 \\
\hline$\%$ of $\Sigma L{ }^{14} \mathrm{C}$ in $\mathrm{TG}$ & 33.7 & 22.6 \\
\hline$\%$ of $\Sigma L{ }^{14} \mathrm{C}$ in $16: 0$ & 4.7 & 27.4 \\
\hline$\%$ of $\Sigma L{ }^{14} \mathrm{C}$ in $18: 0$ & 0 & 7.9 \\
\hline$\%$ of $\Sigma L{ }^{14} \mathrm{C}$ in $18: 2$ & 70.4 & 55.9 \\
\hline$\%$ of $\Sigma \mathrm{L}{ }^{14} \mathrm{C}$ in $20: 4$ & 24.9 & 8.7 \\
\hline
\end{tabular}

Whether the low hepatic free [CoA] or [fatty acids], or a low activity of the synthetase is (are) responsible, the livers of hypothyroid rats 'take up', i.e. esterify and transacylate, $76 \%$ less of an injected dose of labeled 18:2 than do the livers of euthyroid rats (Table 2; Ref. 248). It should be noted that hypothyroidism here sharply decreases reactions that would augment 18:2 acyl contents of membrane PL, yet organelle PL contain excess 18:2 (see Section II.A). This apparent paradox can be ascribed to a greater decrease in reactions that deplete 18:2 acyl contents, such as desaturations. However, the livers of perinatal rats contain little 18:2 while plasma [T3] and [T4] are low-in part, because esterification and transacylation enzymes have yet to develop, perhaps under influences that include thyroid hormones (see Section IV.C).

\section{E. Glycerolipid Synthesis}

Effects of thyroid state on triglyceride synthesis are tissue-specific: in hyperthyroid rats (normals $+\mathrm{LT} 4,0.5 \mu \mathrm{g} / \mathrm{g} /$ day $\times 5$ days) triglyceride synthesis decreases in subcellular fractions from adipose tissue while it increases 2 -fold in those from liver and heart. ${ }^{499}$ In white adipose tissue of mice similarly pretreated with $L T 3$ and injected with $\left[{ }^{3} \mathrm{H}\right] \mathrm{H}_{2} \mathrm{O}$ to detect a triglyceride/fatty-acid cycle from the incorporation of tritium into glycerol and fatty acyls, triglyceride synthesis decreases by about half while fatty acid synthesis is unaffected, showing that cycling is halved. ${ }^{63,64}$ The activities of the adipose tissue enzymes involved (Fig. 2) have not been described in the various thyroid states.

The four enzymatic steps that convert glycerol-3-phosphate and fatty acyls-CoA to triglyceride (B, C, D, and E in Fig. 2) have been studied in liver and heart. The acylation of glycerol-3-phosphate (B in Fig. 2) has been postulated to limit the rates of triglyceride and phospholipid synthesis in normal animals. ${ }^{260}$ However, relative rates, control coefficients, membrane-dependency, or correlations between activity and gene expression, [enzyme], and enzyme covalent modification of organelle enzymes of either tissue in animals in normal and abnormal thyroid states do not appear to have been compared. The increased potential for triglyceride synthesis ${ }^{499}$ is apparently not expressed in intact perfused livers from LT4-pretreated rats, where synthetic rate decreases by $50 \%$, and where making rats hypothyroid accelerates triglyceride synthesis. ${ }^{333,449}$ In vivo LT3-injection similarly decreases synthesis in subsequently prepared hepatocytes. ${ }^{449}$ Perfused livers and isolated hepatocytes are said to reveal those effects of thyroid state, probably due to $\Delta$ [enzyme], that persist in the absence of extrahepatic influence; thus, perfused livers from thyrotoxic animals fail to show the increases in fatty acid and cholesterol synthesis that appear in in vivo and in vitro studies. ${ }^{333}$ On the other hand, hearts of postnatal rats injected daily with T3 30 times incorporate palmitate into triglycerides abnormally slowly. ${ }^{617}$ Because addition of glycerol to liver perfusates or to isolated hepatocytes, ${ }^{449}$ which increases cell [glycerol-3-phosphate], restores the depressed rate to normal in the hyperthyroid preparations, Olubadewo and Heimberg ${ }^{448}$ conclude that decreased availability of glycerol-3-phosphate accounts for the observed decrease in triglyceride synthesis in intact cells, even though activities of synthesizing enzymes increase. Competition by the extremely thyroid-sensitive mitochondrial glycerol-3-phosphate dehydrogenase (see below) would decrease the availability of glycerol-3-phosphate for acylations. Competition for 


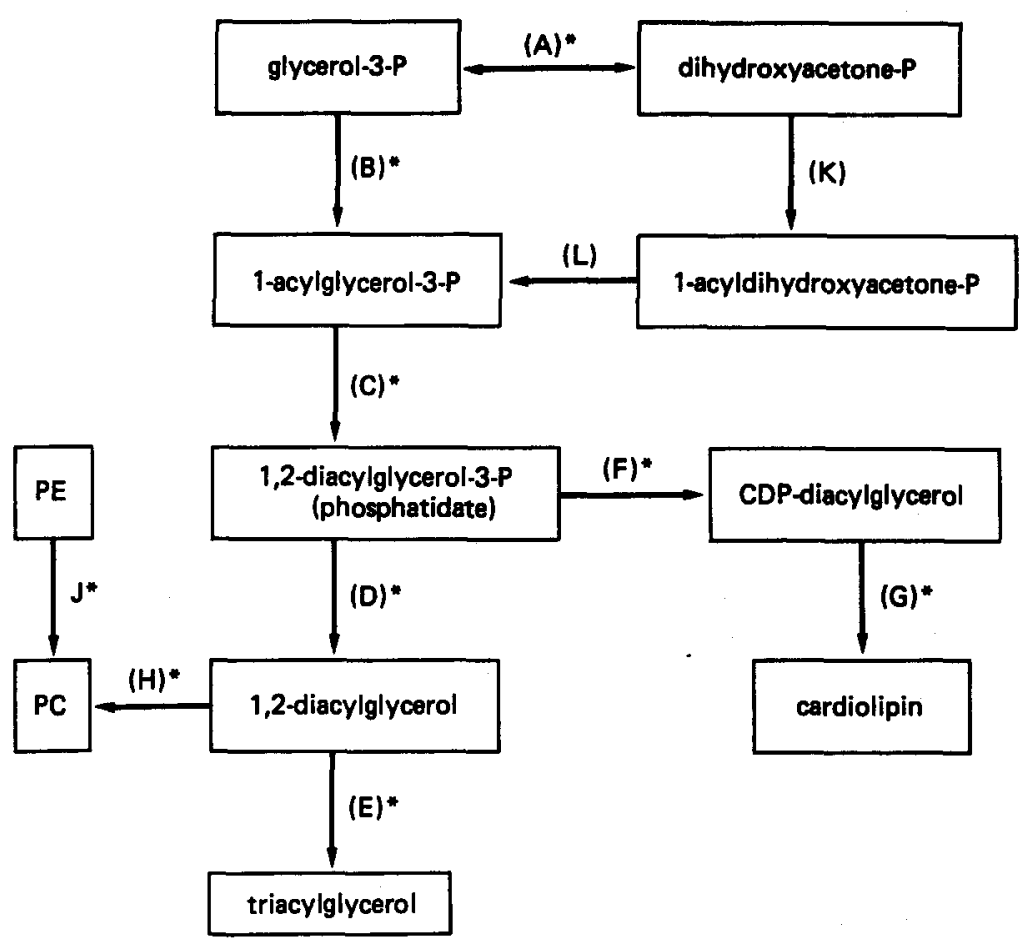

Fig. 2. Effects of thyroid state on some steps in the conversion of glycerol-3-phosphate to triglycerides and phosphoglycerides. $\mathrm{P}=$ phosphate; ${ }^{*}$ affected by thyroid state, others not tested. (A) Glycerol-3-phosphate dehydrogenase; + NAD(H) or FAD $\left(\mathrm{H}_{2}\right)$. (B) Acyl-CoA:glycerol-3phosphate acyltransferase; + acyl-CoA, - CoA. (C) 1-Acylglycerol-3-phosphate acyltransferase; + acyl-CoA, -CoA. (D) Phosphatidate phosphohydrolase; $+\mathrm{H}_{2} \mathrm{O},-\mathbf{P}_{\mathrm{i}}$. (E) Diacylglycerol acyltransferase; +acyl-CoA, - CoA. (F) CTP-phosphatidate cytidyltransferase; +CTP, - PP . (G) Includes phosphatidylglycerophosphate synthetase (+ glycerol-3-P, -CMP), phosphatidylglycerophosphate phosphohydrolase $\left(+\mathrm{H}_{2} \mathrm{O},-\mathrm{P}_{\mathrm{i}}\right)$, and CDP-diglyceride:phosphatidylglycerol phosphatidyltransferase (+CDP-diacylglycerol, -CMP). (H) CDP-choline pathway to PC synthesis, which includes choline kinase, phosphocholine-cytidyl transferase, and phosphocholine diacylglycerol transferase; +choline, +ATP, +CTP, - ADP, - PP, , - CMP. (J) Transmethylation pathway to $\mathrm{PC}$ synthesis, which includes methyltransferase, +S-adenosylmethionine, -S-adenosylhomocysteine. Phospholipase A activities (not shown) can produce FFA at steps B and C. For more complete diagrams of pathways, see Refs $60,388$.

fatty acyls-CoA by the thyroid-sensitive mitochondrial $\mathrm{CPT}_{\mathbf{i}}$ (see Section II.J) would similarly decrease availability of fatty acyls.

Different glycerol-3-phosphate dehydrogenases (A, in Fig. 2) in cytoplasm and in the outer surface of the inner mitochondrial membrane act in a shuttle of substrates that carry reducing equivalents from cytoplasmic $\mathrm{NADH}$ to mitochondrial $\mathrm{FADH}_{2}$. Activity of the $\mathrm{NAD}^{+}$-dependent cytoplasmic enzyme is normally 10 times greater in liver than in heart, and does not change with thyroid state. ${ }^{341,348}$ The FAD-dependent mitochondrial enzyme is normally $40 \%$ more active in heart than in liver. The liver mitochondrial FAD-enzyme responds so robustly to thyroid state that it is used as a verifying marker. Hypothyroidism decreases both liver and heart activity by $-75 \%$, and depresses translocation of reducing equivalents into liver mitochondria. ${ }^{629}$ Thyroid treatment of hypothyroid rats (LT3, $\sim 3 \mu \mathrm{g} / \mathrm{g} 3$ days before killing) or normal rats (thyroid-feeding for 10 days) increases liver mitochondrial activity 20-fold and heart mitochondrial activity 3-fold. PAGE and histochemical identification suggested that the induction does not involve gene expression. ${ }^{607}$ Because treatment with transcription- or translation-blockers inhibits the rise in liver mitochondrial activity, Lee and Miller ${ }^{345}$ concluded that the hormone promotes the supply of mRNA for synthesis of the enzyme. Through the use of polyclonal antibodies to pig brain glycerol-3-phosphate dehydrogenase that react with the rat liver enzyme, Taylor and Ragan ${ }^{601}$ show that T3-treatment of rats concomitantly increases the activity and amount of liver mitochondrial enzyme, and the amount of liver mRNA that codes for the enzyme. 
In livers of rats treated with LT3 for 4 days, microsomal acyl-CoA:glycerol-3-phosphate acyltransferase (B in Fig. 2) activity decreases but mitochondrial activity remains unaffected. ${ }^{104}$ This leaves the increased biosynthesis of triglycerides observed by Roncari and Murthy ${ }^{499}$ unaccounted for, if step B has a high control strength. Hypothyroidism accelerates liver mitochondrial activity $38 \%$, but not the microsomal enzyme. I know of no studies on effects of thyroid on liver enzymes $C$ and $D$ (Fig. 2). Liver microsomal diacylglycerol acyltransferase (E, Fig. 2) activity is thyroid-dependent, being $+60 \%$ above normal in thyrotoxics and $-40 \%$ below normal in hypothyroids ${ }^{647}$ Heart enzymes have been studied more completely. In hearts of rabbits injected with LT3 for 6-10 days, both mitochondria and microsomes form diacylglycerol-3-phosphate from glycerol-3-phosphate and 16:0 fatty acid (steps B, C in Fig. 2, and A in Fig. 1) 4-5 times faster than normally. ${ }^{295}$ T3-treatment accelerates the acyl-CoA:monoacylglycerol-3-phosphate acyltransferase (C, Fig. 2) reaction more than the acyl-CoA:glycerol-3-phosphate acyltransferase (B in Fig. 2) reaction. ${ }^{294,295}$ Further conversion of the diacylglycerol-3-phosphate to neutral glycerides or phosphoglycerides depends on the activities of three sets of reactions that compete for phosphatidate: conversion to triglycerides via $D, E$; to $P C$ and $P E$ via $D, H$; and to cardiolipins via F, G. Phosphatidate phosphohydrolase (D, Fig. 2) activity increases in thyrotoxic rabbit heart microsomes and lysosomes, ${ }^{294,295}$ promoting diglyceride synthesis. Membrane-bound (but not solubilized) phosphohydrolase responds to T3, suggesting thyroid-mediated changes in membrane-dependency. But T3 does not alter heart mitochondrial or microsomal diacylglycerol acyltransferase (E, Fig. 2) activity, ${ }^{295}$ unlike its stimulation of liver enzyme. The increased activities of most of the cardiac triglyceride synthesizing enzymes in cell-free fractions seem consistent with a reported increase in triglyceride content in hyperthyroid guinea pig hearts. ${ }^{58}$ However, no myocardial triglycerides accumulate in hyperthyroid rabbits, ${ }^{295}$ and triglyceride content decreases in hearts of postnatal rats made hyperthyroid. ${ }^{617}$

Thyroid status relates directly to rates of phospholipid synthesis in organelle membranes, and more consistently than to rates of triglyceride synthesis. Hyperthyroidism increases the incorporation of ${ }^{32} \mathrm{P}_{\mathrm{i}}$ injected in vivo into all individual PL of rat liver and kidney mitochondria and into PC and PE of heart, while hypothyroidism decreases incorporation into most of the PL in the mitochondria of liver and kidney but does not affect those of heart. ${ }^{42,596,598,599}$ During the first $6 \mathrm{hr}$ after injecting chickens with $\mathrm{T} 3,{ }^{32} \mathrm{P}_{\mathrm{i}}$ enters liver PC and PE and then the other PL abnormally rapidly. ${ }^{522}$ Hearts of thyrotoxic rats, as compared with those of controls, incorporate more administered fatty acid into $\mathrm{PC}, \mathrm{PE}$, and $\mathrm{CL} ;{ }^{617}$ with the increased formation of diacylglycerol, this suggests that thyroid stimulates pathways to PL synthesis (H, Fig. 2) more than the synthesis of triglycerides (E, Fig. 2).

The major metabolic route to phosphatidylcholine synthesis is from choline $\rightarrow$ phosphocholine $\rightarrow$ CDP-choline + 1,2-diacylglycerol; these steps are included under $\mathbf{H}$ in Fig. 2. This pathway is thyroid-regulated, from studies on the incorporation of injected labeled choline into the PC of liver organelles. In liver mitochondria of hypothyroid rats, uptake decreases to half-normal, in thyrotoxic rats it increases 2- to 3-fold. ${ }^{293}$ In livers of hypothyroid rats that receive one injection of LT3, uptake increases after $10 \mathrm{hr}$ and progresses to 2 -fold by $48 \mathrm{hr}$; choline incorporation into membranes is in the order microsomes $>$ mitochondria $>$ nuclei. ${ }^{596,598,599}$ Recognized thyroid-sensitive steps are the phosphocholine (and -ethanolamine and ceramide) diacylglycerol transferases in liver microsomes. LT3-feeding of rats increases phosphocholine diacylglycerol transferase activity $40 \%$, but induction of hypothyroidism has no effect. ${ }^{647}$ In hypothyroid chicks, activities decline to levels that are $10-15 \%$ of controls; LT4-treatment of these chicks restores all three transferases to normal activity in $36-48 \mathrm{hr} .{ }^{368}$ Cycloheximide given to the hypothyroid chicks restores all activities even faster, in $24 \mathrm{hr}$, and does not alter the LT4 effect. This suggests that blocking synthesis of some protein (a protein kinase or phosphatase?) allows full activity of existing transferase enzymes. The phosphocholine-cytidyl transferase enzyme is inhibited by cAMP-dependent protein kinases. ${ }^{101}$ In contrast to these enzymes, activity of a liver microsomal acyl-CoA :1-acyl- 
glycerol-3-phosphocholine acyltransferase increases in hypothyroidism and decreases in hyperthyroidism. ${ }^{104}$

The minor path for the hepatic synthesis of $\mathrm{PC}$, with a rate-limiting step at the first methylation of PE by methyltransferase I and S-adenosylmethionine in plasma membranes and microsomes (J, Fig. 2), is augmented in the hearts of hyperthyroid rats (see Ref. 101). In vivo incorporation of (methyl $-{ }^{14} \mathrm{C}$ )methionine into $\mathrm{PC}$ and lysoPC is maximally accelerated $5.5 \mathrm{hr}$ after T3-injection. ${ }^{382}$ These methyltransferases are activated by cAMP. dependent protein kinases, reciprocally with the inhibition of the CDP-choline pathway. ${ }^{101}$ Because PC synthesized via transmethylation contains more unsaturated fatty acyl groups than PC synthesized via CDP-choline transfer, a differential sensitivity of these paths to thyroid levels may produce some of the observed alterations in PC unsaturation, ${ }^{248}$ and perhaps modulate receptor responses. ${ }^{101}$

In CL synthesis, step F and the several steps in G (Fig. 2) are accelerated in rats made thyrotoxic with 7 large daily doses of T4 (K. Y. Hostetler, personal communication). Hepatic CL synthesis is completely in the mitochondrial inner membrane. ${ }^{106,110,253,254,573}$ The required liponucleotides are produced by mitochondrial or microsomal CTP-specific transferases from phosphatidate (F, Fig. 2) ${ }^{482}$ The T4-treatment increases mitochondrial CTP-phosphatidate cytidyltransferase activity $101 \%$ and mitochondrial phosphatidylglycerophosphate synthetase activity $41 \%$. Activity of the mitochondrial phosphatidylglycerophosphate phosphohydrolase was not measured, but is generally thought not to limit CL synthesis. T4 accelerates synthesis of CL by the CDP-diglyceride:phosphatidylglycerol phosphatidyltransferase by $35 \%$. If thyroid treatment stimulates CL synthesis, how then does hypothyroidism increase the CL/PL ratio in rat liver mitochondria? Hypothyroidism usually augments the concentration of a metabolic intermediate by decreasing its degradation more than its synthesis, not by increasing its synthesis. Although 18:2 acyls normally turn over slightly faster in liver CL than in other PL, CL turns over much more slowly than other $\mathrm{PL},{ }^{337}$ and $\mathrm{CL}$ and mitochondrial half-lives are comparable. CL is degraded by phospholipases $\mathrm{A}$ and perhaps $\mathrm{D}$, but not $\mathrm{C}$; $\mathrm{Ca}^{2+}$-ions activate a mitochondrial phospholipase $\mathrm{A}_{2}$ that readily acts on $\mathrm{CL}$ in situ, although less than on PC, PS, or PE. ${ }^{253}$ Brief T4-treatment enhances hepatic phospholipase $A_{2}$ activity, ${ }^{255,406}$ and a reciprocal suppression in hypothyroidism might depress 2-deacylation in CL more than in the other PL. Lysosomal hydrolases ultimately degrade CL, but do not appear to have been examined for thyroid-dependence. Alternatively, and depending on the substrate turnover rates of steps $\mathrm{F}+\mathrm{G}$ vs. D (Fig. 2), the phosphatidate phosphohydrolase might be a metabolic branch-point where lack of thyroid hormone increases phosphatidate $\rightarrow C L$ conversion by decreasing diglyceride synthesis more.

The mechanisms whereby cardiolipins from heart, liver and kidney (but not brain) are normally so enriched in 18:2 acyls are not known. Acyltransferases reacylate dilyso-CL with substrate specificities 18:0-CoA $>18: 1-\mathrm{CoA} \gg 18: 2-\mathrm{CoA},{ }^{253}$ yet the labeled 18:2 acyl moieties of CL turn over more rapidly than other CL fatty acyls ${ }^{337}$ and still maintain their profusion. Hyperthyroidism depletes CL 18:2 acyls (see Section II.A) presumably by stimulating the desaturative conversion 18:2-CoA $\rightarrow 20: 4-\mathrm{CoA}$ (see Section II.I), but then it is not clear why hypothyroidism does not increase CL 18:2 acyls since it retards this conversion (unless 18:2 content is already maximal). These mechanisms are pertinent to thyroid influences on membrane content of $\mathrm{CL}$ and $\mathrm{CL}$ content of 18:2 acyls, and on oxidative phosphorylation, that are discussed in Section IV.

\section{F. Lipolysis}

LT4-injection into normal rats stimulates hepatic phospholipase $A_{2}$ activity in 2 days, as well as the oxidative conversion of a small fraction of the liberated 20:4 acyls to prostaglandins (see Section II.K). No studies on hypothyroid rats are on hand. Since these enzymes selectively remove PUFA from $s n-2$ positions of PL, the simultaneous thyroid stimulation of acyltransferases (B, $C$ in Fig. 2) should increase exchange and remodeling ${ }^{338,369,606}$ of PL fatty acyl groups. 
Adequate levels of thyroid hormone are permissive for epinephrine-induced but not basal unstimulated lipolysis of triglycerides in adipose tissue; ${ }^{14,576}$ hyperthyroidism triples both basal and epinephrine-stimulated lipolysis; one injection of T3 into a euthyroid rat has no effect at $3 \mathrm{hr}$, but 4 injections over $12 \mathrm{hr}$ maximize the lipolytic response to epinephrine. ${ }^{112}$ Thyroid exerts these effects via the responsiveness of lipolysis to [epinephrine]: hypothyroidism lowers sensitivity to the point that maximal lipolytic rates require 3 times more epinephrine than do euthyroid preparations, but the maximae are similar; $16 \mathrm{hr}$ after T3-injection of hypothyroid rats, epinephrine-sensitivity improves. ${ }^{176}$ Thus, hypothyroidism does not appear to reduce the amount or capacity of lipolytic apparatus, although the amount of hormone-sensitive lipase does not appear to have been measured directly. Since the lipolytic effects of other hormones (e.g. norepinephrine, ACTH, TSH, glucagon, vasopressin) also depend on the thyroid state, it seems that thyroid regulates through effects on [cAMP], the phosphorylation state of the hormone-sensitive lipase (see Section II.F and III.B), and/or the properties of cell membrane receptors (see Section III).

\section{G. Ketogenesis}

Ketogenesis from 18:1 oxidation (steps A, C, G, H and I in Fig. 1) increases in perfused livers ${ }^{333,411}$ and in hepatocytes ${ }^{448}$ from LT3-pretreated rats. Livers from hypothyroid rats either make more ketone bodies but oxidize less $18: 1$ to $\mathrm{CO}_{2}{ }^{233}$ or show no change from normal. ${ }^{411}$ The HMG-CoA pool destined for ketogenesis is mitochondrial, that for cholesterogenesis cytosolic, and the HMG-CoA synthases are compartmented accordingly. The mitochondrial HMG-CoA synthase may be 'rate-limiting' for ketogenesis (see Ref. 650), but data are not available on its activity and that of the HMG-CoA lyase in different thyroid states.

\section{H. Cholesterol Metabolism}

A variety of interacting physiological stimuli, including thyroid state, regulate cholesterol metabolism. ${ }^{68,394,428}$ Thyroid-sensitive sites in cholesterol metabolism include steps in intracellular synthesis, conversions to other sterols, cholesterol processing at cell membranes, and cholesterol transport.

\section{Intracellular Cholesterol Metabolism}

The rate of cholesterol synthesis from acetate (but not from mevalonate) and the turnover of cholesterol relate directly to thyroid levels. ${ }^{122}$ The hepatic microsomal $\beta$-hydroxy- $\beta$-methylglutaryl-coenzyme A reductase (HMGR; $J$ in Fig. 1 ) is said to be 'rate-limiting' for acetyl-CoA $\rightarrow$ cholesterol. This enzyme is highly regulated, by hormones, and by cholesterol and its precursors and metabolites. Cholesterol or its oxidized products decrease cholesterol synthesis by suppressing transcription of the HMGR gene; LDL, mevalonate, 25-hydroxycholesterol, and phosphorylation of HMGR accelerate HMGR degradation. In thyrotoxic rats, HMGR, acetate thiokinase, and HMG-condensing enzyme activities all increase over normal rates; in hypothyroids only HMGR activity decreases, and to very low levels. ${ }^{190}$ Experiments on the effects of thyroid treatment under different conditions give complementary information on the time course of the activation and later induction of HMGR. Perfusion of isolated livers of hypothyroid rats with T3 activates HMGR within $30 \mathrm{~min}$, 5-fold at $120 \mathrm{~min}$ and 10 -fold at $360 \mathrm{~min} .{ }^{190}$ One injection of T3 into hypothyroid rats induces HMGR: activity is unchanged when the earliest time of measurement is $12 \mathrm{hr}$, and begins to rise only at $36 \mathrm{hr}$, to reach a maximum at $48 \mathrm{hr} .{ }^{197}$

Thyroid treatment induces HMGR even more strikingly in hypophysectomized rats, but as slowly as in hypothyroid rats. ${ }^{429}$ The liver microsomal HMGR activity in untreated animals is depressed more than the $50 \%$ seen in hypothyroids, to barely detectable levels. A dose of LT3 that saturates $90 \%$ of liver nuclear T3-receptors increases activity only after 
$36 \mathrm{hr}$, and maximally at $48-72 \mathrm{hr}$; HMGR activity in rough microsomes rises to 4 times normal, and acetate is converted to cholesterol at 10 times the normal rate. Under these circumstances, thyroid hormone induces HMGR only in liver and adrenal, and not in kidney, brain, or testis. T3-treatment of normal rats does not activate. Inhibitor studies indicate that these relatively slow inductions in hypophysectomized rats involve new mRNA synthesis. ${ }^{121,429}$ Forty-eight to $72 \mathrm{hr}$ after T3-injection, reductase mRNA appears together with a $97 \mathrm{kDa}$ protein that reacts with antisera to pure HMGR, and reductase activity accelerates many-fold. ${ }^{520}$ An HMGR-immunoreactive, $35 \mathrm{kDa}$ protein is present in untreated hypophysectomized rats and is not augmented after T3-treatment. The lag period of $36 \mathrm{hr}$ practically eliminates the possibility that T3-occupancy of nuclear receptors is directly promoting HMGR gene expression (see Section II.B.2), and indicates that the increased activity at $3 \mathrm{hr}$ of liver perfusion is an activation of existing enzyme. It seems likely, then, that synthesis of new HMGR is mediated through secondary alterations after T3 enters the cell. Zammit and Easom ${ }^{652}$ propose that covalent modification of HMGR mediates $\Delta[H M G R]$ : phosphorylated enzyme is inactive and is proteolytically degraded faster than the active dephosphorylated form. ${ }^{467}$ This would explain the sequence activation $\rightarrow$ persistence but not activation $\rightarrow$ synthesis.

Injection of a glucocorticoid blocks the T3-promoted increase in activity ${ }^{334}$ and the appearance of $97 \mathrm{kDa}$ active HMGR, and halves the amount of new reductase mRNA; 520 perhaps the absence of ACTH in hypophysectomized rats permits T3-induction to exceed that in hypothyroid rats. Feeding mevinolin or cholestyramine to these animals increases HMGR activity to levels only $10-40 \%$ of those reached after T3-injection. It is concluded that T3 is not absolutely required, and may induce HMGR indirectly, perhaps via oxysterol metabolites of mevalonate. Thyroid also activates HMGR in concert with insulin or fasting/refeeding. In hypophysectomized diabetic rats, the activity that insulin induces at $2 \mathrm{hr}$, and that T3 induces at $54 \mathrm{hr}$, are each blocked by administration of glucagon or glucocorticoids ${ }^{334}$-perhaps indicating that cAMP-mediated protein-phosphorylation blocks both enzyme activation and gene expression. These studies may be pertinent to possible thyroid effects through [cAMP] regulation (see Section III.B).

Most investigators agree that dephosphorylation activates, and phosphorylation deactivates, the HMGR in situ in microsomes. ${ }^{29,266,268,652}$ Some find that phosphorylation reversibly inactivates purified, electrophoretically homogeneous HMGR, ${ }^{427,432}$ others that it does not. ${ }^{428}$ Ness et al. ${ }^{428,430,431}$ suggest that a mevalonate kinase, present in preparations of HMGR kinase, accounts for effects attributed to the HMGR kinase, and that the allosteric kinetics of NADPH as cofactor for the double reduction of HMGCoA may mediate regulatory effects. Thyroid state controls cytoplasmic [NADPH] (see Section II.C). The significance of HMGR regulation by such covalent modification has been questioned because the enzyme was found to be $>90 \%$ phosphorylated and inactive under most conditions, including those favoring cholesterol synthesis (see Ref. 652). However, with appropriate rapid methods (using cold-clamped livers from anesthetized fed rats), $80 \%$ of HMGR is in dephosphorylated, active form, and insulin-state in vivo acutely regulates HMGR phosphorylation. Analogous specific effects of thyroid-state on HMGR phosphorylation await similar examination, even though T3-administration takes days to detectably augment protein kinase activities and a general phosphorylation of endogenous proteins (see Section II.B.2). Thyroid also regulates the adenylate kinase and thereby [cAMP] (see Section III.B.1), which may in turn modulate protein kinase or phosphatase activities.

The HMGR is membrane-dependent. Arrhenius profiles of its activity inflect, when measured in microsomes, ${ }^{401,472,558,562 \mathrm{a}}$ and in a partly purified preparation. ${ }^{427}$ Alterations of endoplasmic reticulum fatty acyl and cholesterol composition, produced by diet, or by inducing diabetes (thyroid effects have not been examined), shift the transition temperatures and alter $E_{\mathrm{a}}$ values. ${ }^{562 \mathrm{a}}$ Compared with rats fed saturated fatty acids, the feeding of $18: 2$ fatty acid doubles the 18:2 content of the endoplasmic reticulum membrane, decreases HMGR activity at $37^{\circ} \mathrm{C}$ by $70 \%$, and doubles acyl-CoA:cholesterol acyltransferase activity (Ref. $401 ; \mathrm{K}$ in Fig. 1). Adding cholesterol to either the saturated fat 
or the $18: 2$ diet decreases HMGR activity by $90 \%$. It has been suggested that membrane cholesterol rather than fatty acyls modulates HMGR activity, because the increased $E_{\mathrm{a}}$ above the transition temperature indicates decreased fluidity. ${ }^{401}$ The opposing effects on acyl-CoA:cholesterol acyltransferase activity are ascribed to its location in the rough endoplasmic reticulum, while the HMGR is in the smooth endoplasmic reticulumalthough cholesterol accumulation does not seem to have been so localized. Studies comparing normal and leukemic lymphocytes ${ }^{472}$ also conclude that endoplasmic reticulum membrane cholesterol regulates HMGR activity. If the general inhibition of lipogenesis by high contents of $18: 2$ acyls in endoplasmic reticulum ${ }^{279,413}$ includes inhibition of cholesterol synthesis, T3-injection could produce early activation of HMGR through the observed rapid depletion of ER 18:2 acyls, in a period when cholesterol/fatty acyl ratios stay constant. ${ }^{236,248}$

Cholesterol $7 \alpha$-hydroxylase (L in Fig. 1), an NADPH-dependent cytochrome P450 (perhaps a specific P450) of the endoplasmic reticulum, catalyzes 'the rate-limiting, committal step' in hepatic bile acid biosynthesis, and is thus a major disposal path for cholesterol. The enzyme is a regulatory site: it has a short half-life $(2-4 \mathrm{hr})$ and a diurnal rhythm, and is rapidly activated by feeding carbohydrate to starved animals. Almost all physiological regulators, including thyroid hormones, act on the $7 \alpha$-hydroxylase in coordination with, and in the same direction as, their actions on the HMGR, the acyl-CoA:cholesterol acyltransferase, and the cholesterol ester-hydrolase. Hypothyroidism decreases activity. ${ }^{582}$ Thyroid treatment activates in hypophysectomized rats, and so acts independently of those hormones evoked by pituitary trophins. However, thyroid state also modulates other regulators of the hepatic cholesterol $7 \alpha$-hydroxylase.

The cholesterol $7 \alpha$-hydroxylase is more sensitive to in vivo thyroid levels than is the HMGR. ${ }^{592}$ In fasted euthyroid rats, refeeding with glucose activates cholesterol synthesis within $3 \mathrm{hr}$, and increases $7 \alpha$-hydroxylation starting $1 \mathrm{hr}$ later. ${ }^{593}$ When rats are made increasingly hypothyroid with graded doses of propylthiouracil, and then fasted and refed, the lowest dose decreases stimulation of $7 \alpha$-hydroxylase activity, but only the highest dose decreases activation of acetyl-CoA conversion to fatty acids and to cholesterol. Conversely, making rats hyperthyroid with graded doses of T4 increases glucose-induced $7 \alpha$-hydroxylase activity at the lowest T4-dose, cholesterol synthesis at the highest. Further, glucose stimulates $7 \alpha$-hydroxylation more rapidly in hyperthyroid than in hypothyroid preparations, but activates cholesterol synthesis at about the same time in both.

The mechanisms of the early regulations are consistent with an activation by dephosphorylation, except for the adrenal cortex cholesterol esterase which is inactivated thereby, and the liver enzyme which does not appear to be affected at all. However, the $7 \alpha$-hydroxylase has only been studied through activity changes in microsomal preparations incubated with protein kinase $+\mathrm{MgATP}$, or protein phosphatase. ${ }^{178,331,524}$ No measurements of the phosphorylation stoichiometry of the purified enzyme are on hand, so the $7 \alpha$-hydroxylase does not as yet meet the criteria of Krebs and Beavo ${ }^{319}$ for an enzyme regulated by phos/dephos. Nor have the rapid assays used by Zammit and Easom ${ }^{652}$ on the HMGR been applied to this enzyme.

The details of the modulation of the cholesterol $7 \alpha$-hydroxylase differ from those of the HMG-CoA reductase in several ways. Insulin administration, perhaps by way of a specific phosphoprotein phosphatase, activates the reductase but not the hydroxylase; hydrocortisone activates the hydroxylase but deactivates the reductase. Possibly the different mechanisms are involved in what have seemed to be 'biphasic actions' of the administered thyroid hormones ${ }^{226}$ on cholesterol levels in cells and blood, in which low T3-dosage raises cholesterol levels and high T3-dosage lowers them.

Thyroid hormones also promote steps in the synthesis of bile acids from $7 \alpha$-hydroxycholesterol. ${ }^{332.414}$ In rats, but not in humans, the hormone increases [chenodeoxycholic acid] at the expense of [cholic acid], apparently by activating the microsomal (but not the mitochondrial) 26-hydroxylase, and deactivating the 12 $\alpha$-hydroxylase. Hypothyroidism effects the opposite changes. These modulations are not general to cytochrome P450s, since 
thyroid treatment increases, and hypothyroidism decreases, total liver P450. Such selectivity also appears in the undisturbed ability of the hypothyroid rat to induce specific hepatic P450s after treatment with methylcholanthrene, ${ }^{243}$ phenobarbitone and other xenobiotics. ${ }^{41}$

Thyroid state affects steroid kinetics in the whole body. In thyrotoxic humans, secretory rates of the C-21 steroids cortisol and aldosterone are augmented, as is their metabolic clearance which includes a shorter half-life and increased turnover. ${ }^{184}$ Hypothyroidism exhibits opposite effects. Contrarily, thyrotoxicosis depresses metabolic clearance and secretory rates of the C-19 and C-18 steroids testosterone and estradiol, while hypothyroidism increases them. These phenomena seem to be caused in part by the thyroid-dependence of specific, high-affinity, binding proteins in plasma of males and females. The [free steroid] levels in plasma are not changed, nor are symptoms of steroid excess or deficiency obvious. Thyroid levels do affect cellular metabolism however. Thyroid increases aromatase activity. An increase in hepatic reduction of $\mathrm{C}=\mathrm{C}$ bonds in ring $A$ is attributed to an increase in [NADPH] (see Section II.C) rather than to induction of the $\Delta 4$-corticosteroid hydrogenase. ${ }^{645}$ Thyroid also accelerates $5 \alpha$-reductions and $17 \beta$-hydroxysteroid dehydrogenations. ${ }^{184}$ Sex difference regulates thyroid effects on progesterone metabolism in hepatic microsomes. ${ }^{298}$ In males, progesterone hydroxylation is normally faster, and $\Delta 4$-reduction slower, than in females. T4-treatment decreases hydroxylation and increases $\Delta 4$-reduction in males but not in females.

Intracellularly, the lysosomal acid cholesterol esterase activity in liver and adipose tissue (but not in heart) is thyroid-dependent: ${ }^{547}$ hypothyroidism decreases activity, T3-treatment for 3 days increases it.

\section{Cholesterol Transport}

Thyroid hormones reduce plasma [cholesterol] at least in part through increased hepatic catabolism of cholesterol. ${ }^{397}$ The hypocholesterolemic effects of thyroid hormones offer a therapeutic opportunity for prevention and even reversal of atherosclerosis. However, the strong inotropic and chronotropic cardiac effects of LT4 and LT3 preclude their use where most needed, in coronary atherosclerosis. DT4 lowers [cholesterol] and stimulates heart action less than LT4 but still formidably. A recently described T3-analogue, a synthetic thyromimetic SK\&F-94901, lowers plasma [cholesterol] in hypothyroid rats as effectively as LT3 by acting on liver cholesterol metabolism but has a strikingly lesser cardiac chronotropic effect; its mechanism of action is described in Section II.B.

Thyroid hormones participate in the regulation of several steps in the pathways described by Bilheimer ${ }^{38}$ as transporting exogenous or endogenous cholesterol, and those of reverse transport. In the endogenous transport, hypothyroidism decreases the expression of hepatic plasma membrane LDL-receptors, and the decreased LDL degradation contributes to the observed hypercholesterolemia; ${ }^{530} \mathrm{~T} 3$ treatment restores receptormediated catabolism of LDL..$^{74}$ In the reverse pathway, the PC-cholesterol acyltransferase, which is thought to esterify cholesterol in plasma, is poorly secreted from hepatocytes of hypothyroid rats, further contributing to the increased [cholesterol]..$^{209,493}$ In the exogenous path, it has been proposed that the intestine plays a major role in cholesterol metabolism. ${ }^{387}$ Thyroid state-altered rates of absorption, synthesis and excretion are said to be offset by compensatory changes in cholesterol transformation, to maintain the cholesterol balance. These workers disagree with the usually accepted idea that continuous thyroid treatment decreases blood cholesterol concentration by stimulating cholesterol degradation and elimination more than cholesterol synthesis. If this were so, they point out, such treatment should progressively deplete body cholesterol-but measured body cholesterol content stays constant in hyperthyroidism. They show that hyperthyroidism depresses the coefficient of intestinal absorption of cholesterol (the amount of dietary cholesterol absorbed/ingested), which promotes biosynthesis and results in large increases in fecal excretion of cholesterol and products. Conversely, hypothyroidism increases the absorption coefficient, decreases fecal excretion, and thereby depresses biosynthesis. No mech- 
anisms for this homeostasis are specified, but presumably they would involve effector actions of cholesterol and metabolites on synthesis and oxidations.

Thyroid dysfunction is associated with altered compositions and concentrations of all major plasma lipoprotein classes. ${ }^{209,305}$ The lipoprotein lipase that heparin releases into blood from extra-hepatic capillary endothelium is involved in all three pathways of plasma cholesterol metabolism. Its activity decreases in severe hypothyroidism and normalizes after hormone treatment. Conflicting results are reported in thyrotoxicosis, but Hansson et $a{ }^{209}$ find no change in human plasma activity, where plasma [cholesterol] decreases $20 \%$ through depressed [LDL] and total [HDL]; it is not clear what lipoprotein lipase hydrolysis of glycerides and the subsequent concentration of cholesterol into IDL particles contribute to plasma [cholesterol]. The role of the hepatic cell membrane lipase in plasma lipoprotein metabolism is also not well understood, but heparin releases less than normal lipase activity into the blood stream in hypothyroid subjects and more than normal in euthyroids treated with T3 ${ }^{209.263}$ Feeding hypothyroid rats a diet enriched in 18:2 and 20:4 restores levels of heparin-released hepatic lipase in a week, suggesting that depression of liver cell membrane fluidity or $\omega 6$-PUFA content is responsible for defective binding and/or content of this enzyme. ${ }^{263}$

\section{Fatty Acid Desaturation}

Oxygen-dependent liver microsomal fatty acyl-CoA desaturases specific for introducing a double bond into the $\Delta 9$ or $\Delta 6$ positions (and probably the $\Delta 5$ and $\Delta 4$ as well) share an electron-donating transport system that consists of NADH-cytochrome $b_{5}$ reductase and cytochrome $b_{5}$. In microsomes from hypothyroid rats, the $V$ and $K_{\mathrm{m}}$ for the reductase, measured with cytochrome $c$ as electron acceptor, are at normal levels. ${ }^{243}$ Cytochrome $b_{5}$ content is $50 \%$ above normal in microsomes from hypothyroid rats, $30 \%$ below normal in those from thyrotoxic animals, and is transiently decreased hours after hypothyroids are injected with T3 (Table 3). However, the thyroid dependency of the desaturase system resides in the desaturase proteins, as shown by spectrophotometric assays of the rates of reoxidation of reduced $b_{5}$ in the presence of the acyl-CoA substrate. ${ }^{450,457,458}$ Apparent first-order rate constants, $k$, are calculated from initial rates; $k^{+}$is the rate constant attributable to electron transport through the substrate, and $k^{-}$is the cytochrome $b_{5}$ autoxidation rate constant observed in the absence of substrate (Table 3). In hypothyroidism, $k^{-}$is at normal levels; T3 injected into hypothyroid rats progressively raises $k^{-}$over $4 \mathrm{hr}$; in T3-injected normal (thyrotoxic) rats $k^{-}$is high.

TABle 3. Fatty Acyl-CoA Desaturase Activities, Cytochrome $b_{5}$ Autoxidation Rates and Contents in Rat Liver Microsomes, and Changes after LT3 Injection(s)*

\begin{tabular}{|c|c|c|c|c|c|c|c|}
\hline & \multirow{2}{*}{$\begin{array}{c}\text { Controls } \\
(6-8)\end{array}$} & \multirow{2}{*}{$\begin{array}{l}\text { Hypothyroids } \\
(6-10)\end{array}$} & \multicolumn{4}{|c|}{$\begin{array}{l}\text { Hypothyroids + LT3 } \\
\text { (hours after injection) }\end{array}$} & \multirow{2}{*}{$\begin{array}{l}\text { Controls } \\
+ \text { LT3 (8) }\end{array}$} \\
\hline & & & $0.5(2)$ & $1.0(6)$ & $2.5(5)$ & $4.0(6)$ & \\
\hline \multicolumn{8}{|l|}{$\Delta 9$-Desaturase: } \\
\hline $\begin{array}{c}K_{\max }(\mu \mathrm{M}) \\
\Delta 6-\text { Desaturase: }\end{array}$ & $\begin{array}{l}2.7 \\
2.0\end{array}$ & $\begin{array}{l}3.1 \\
1.6\end{array}$ & $\begin{array}{l}3.7 \\
1.9\end{array}$ & $\begin{array}{l}4.9 \\
0.7\end{array}$ & $\begin{array}{l}5.6 \\
1.2\end{array}$ & $\begin{array}{l}8.9^{A} \\
3.3\end{array}$ & $\begin{array}{c}10.2^{\mathrm{A}} \\
5.3\end{array}$ \\
\hline$k_{\max }\left(\min ^{-1}\right)$ & 4.5 & $2.2^{\mathrm{C}}$ & 6.1 & $4.4^{C}$ & $6.0^{A}$ & 3.5 & 2.8 \\
\hline $\begin{array}{c}K_{\mathrm{m}}(\mu \mathrm{M}) \\
\Delta 5 \text {-Desaturase: }\end{array}$ & 17.9 & 3.6 & 9.0 & 10.2 & 9.7 & 5.1 & 2.6 \\
\hline$V(\mathrm{pmol} / \mathrm{min} / \mathrm{mg})$ & 12.8 & 7.0 & & & & & 26.0 \\
\hline $\begin{array}{c}K_{\mathrm{m}}(\mu \mathrm{M}) \\
\text { Autoxidation: }\end{array}$ & 0.15 & 0.06 & & & & & 0.14 \\
\hline$k^{-}\left(\min ^{-1}\right)$ & 1.9 & 1.6 & 2.2 & $2.4^{\mathrm{B}}$ & $2.4^{\mathrm{B}}$ & $2.9^{\mathrm{B}}$ & $4.2^{\mathrm{B}}$ \\
\hline Cyt. $b_{5}(\mathrm{nmol} / \mathrm{mg})$ & 0.13 & $0.20^{\mathrm{a}}$ & 0.20 & 0.17 & $0.14^{B}$ & 0.18 & $0.09^{8}$ \\
\hline
\end{tabular}

*See Table 1 for details, and the text for definitions of kinetic parameters. Only means are shown; for 42 values, the coefficient of variation is $21.1 \% \pm 2.1 \%$ SE. For group comparisons: $p<{ }^{\mathrm{aA}} 0.001 ;{ }^{\mathrm{bB}} 0.01$; ${ }^{\mathrm{CC}} 0.05$; lower case symbols denote $p$ values for comparisons of Hypothyroids vs. Controls, upper case symbols for comparisons of Hypothyroids + LT3 vs. Hypothyroids and Controls + LT3 vs. Controls. 
The extrapolated maximal desaturation rate constant, $k_{\max }=k^{+}$at $[\mathrm{S}]=\infty$, and $K_{\mathrm{m}}$ for the $\Delta 9$-desaturase (stearoyl-CoA, $37^{\circ} \mathrm{C}$ ) in fasted hypothyroid rats is the same as in fasted euthyroid rats; $k^{+}$is 4 times normal in fasted thyrotoxic rats (Table 3). In hypothyroid animals a fasting/refeeding-with-carbohyrate cycle does not increase the $\Delta 9$-desaturase $k^{+}$, whereas in normal rats it raises $k^{+}$strikingly. ${ }^{243,458}$ One LT3 injection increases the $\Delta 9$-desaturase $k^{+}$in fasting hypothyroids linearly over $4 \mathrm{hr}$ (Table $3, r=0.972$ ) and (not shown) to 5-fold at $17 \mathrm{hr}$. The T3-induced changes in microsomal PL fatty acyl composition ${ }^{248}$ do not appear to mediate the increased $k^{+}$, as judged from Arrhenius profiles. ${ }^{243}$ Brain $\Delta 9$-desaturase activity decreases from birth, to reach $10 \%$ of that in the fetus at maturity; fasting/refeeding does not consistently induce the adult enzyme. ${ }^{644}$

The purpose of the thyroid dependency of this enzyme is likely connected with the thyroid dependency of the enzymes of de novo fatty acid synthesis, which also are activated 1-4 hr after LT3 injection in hypothyroid rats, and are subsequently synthesized (see Section II.B). In view of the activations of acetyl-CoA carboxylase and the fatty acid synthetase by dephosphorylation, it is therefore of some interest that $2 \mathrm{hr}$ incubation of Tetrahymena pyriformis with $\beta$-adrenergic agonists increases [cAMP] together with fatty acid synthesis and the activities of the microsomal $\Delta 9$ - and $\Delta 12$-desaturases. ${ }^{613}$ The dietarily 'induced' $\Delta 9$ enzyme in rat liver microsomes is partly and progressively inhibited by a protein kinase + ATP (unpublished data), further suggesting that the $\Delta 9$-desaturase acts in concert with the fatty acid synthesizing process. Unlike the cytosolic fatty acid synthesizing enzymes, the $\Delta 9$-desaturase has not yet been resolved from other microsomal polypeptides for study of its phosphorylation state or for immunoassay.

Three metabolic roles can be distinguished for the $\Delta 9$-desaturase in converting 18:0-CoA $\rightarrow$ 18:1-CoA. Derived (as well as dietary) 18:1-acyls are normally a major membrane component. Oleoyl-CoA becomes a precursor for PUFA in membranes, mainly $20: 3 \omega 9$ produced by the $\Delta 6$ - and $\Delta 5$-desaturases, only when $\omega 6$-EFA intake is deficient: the $\Delta 9$ enzyme is the first step in producing unsaturated fatty acids from endogenous sources when necessary. The $\Delta 9$-desaturase is proposed as a major locus where dietary linoleate down-regulates hepatic lipogenesis, ${ }^{279} 18: 2$ also selectively damps the enzymes of de novo fatty acid synthesis. ${ }^{413}$ While dietary carbohydrate rapidly induces the enzyme, dietary $18: 2$ inhibits the $\Delta 9$-desaturase even more effectively and rapidly than it inhibits the fatty acid synthetase, the $\Delta 9$ enzyme having the much shorter biological half-life of 3-4 hr. Insulin induces $\Delta 9$-desaturase activity when incubated for $48 \mathrm{hr}$ with hepatocytes. ${ }^{280}$ In diabetic rats, activity stops in microsomes of liver and fat; fasting/refeeding induces the enzyme only $24 \mathrm{hr}$ after insulin treatment. ${ }^{168}$ Interestingly, thyroid treatment of diabetic rats induces the $\Delta 9$-desaturase and fatty acid synthesis independently of insulin. ${ }^{175}$

Liver microsomal $\Delta 6-, \Delta 5$ - and $\Delta 4$-acyl-CoA desaturases, ${ }^{56,278,570}$ and separate microsomal desaturases that act on the corresponding PL-acyls rather than acyl-CoAs as

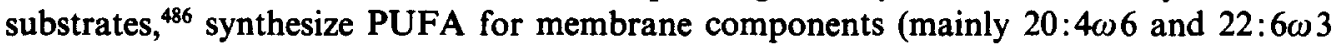
in rat organelles) and as precursors of prostanoids. In hypothyroids, the acyl-CoA $\Delta 6-$ and $\Delta 5$-desaturase $k_{\max }$ values are about half-normal ( $p>0.05$ for the $\left.\Delta 5-V\right)$; values for $K_{\mathrm{m}}$ are normal ( $p>0.05$ for the low $\Delta 6$-desaturase $K_{\mathrm{m}}$ ) (Table 3). Injecting hypothyroid rats with LT3 transiently increases the $\Delta 6$-desaturase $k_{\max }$ about 3-fold in $2.5 \mathrm{hr}$. Thyrotoxicosis doubles the $\Delta 5$-desaturase $k_{\max }$, and leaves the $\Delta 6$-desaturase $k_{\max }$ unaltered. The high $\Delta 6$-desaturase activity in the thyroid-sensitive brain of fetal rats persists to 20 days after birth, as the brain becomes less responsive to thyroid, ${ }^{94}$ in consistence with the behavior of the hepatic enzyme.

The thyroid-dependence of the hepatic $\Delta 6$ - and $\Delta 5$-desaturases seems to account for the 18:2 acyl accumulation in organelle membranes of hypothyroid rats, and depletion in thyrotoxics. ${ }^{140,141,236,239,242,262}$ It accounts as well for decreased ability of the livers of hypothyroid rats to convert administered $\left[1-{ }^{14} \mathrm{C}\right] 18: 2$ fatty acid to $20: 4$ fatty acyls (Table 2). Livers of diabetic rats show a similar defect, ${ }^{158}$ but no insulin-independent $T 3$-induction of the $\Delta 6$ - and $\Delta 5$-desaturases has been described that parallels the induction of the $\Delta 9$-desaturase. ${ }^{175}$ The transience of the restoration of $\Delta 6$-desaturase activity after hormone injection (Table 3 ) suggests either enzyme turnover or successive activation and 
deactivation. Despite the restored $\Delta 6$-desaturation activity, which should improve the $18: 2 \rightarrow 20: 4$ conversion (unless the $\Delta 5$-desaturase proves to be unrestored by $\mathrm{T} 3$ ), T3-administration counter-correctively depresses PUFA contents in mitochondrial and microsomal lipids even below the abnormally low levels in untreated hypothyroid rats (Ref. 248; Table 1). The most likely concomitant T3-induced mechanism to remove PUFA is massive and rapid substitution by newly synthesized saturated and mono-unsaturated fatty acyls (see Section II.B.1), like that after fasting/refeeding. ${ }^{7.8}$ Phospholipase $A_{2}$ action (see Section II.F) may remove PUFA.

\section{Anuran Metamorphosis}

Thyroid-induced metamorphosis involves early general increases in hepatic protein synthesis, and phospholipid synthesis increases even earlier-presumably to provide membranes to support the increased translation of newly expressed mRNA. ${ }^{596-599}$ We have recently found that thyroid control over PUFA composition is also involved very early in amphibian metamorphosis.

Tadpoles are naturally athyroid. Lipids of tadpoles and frogs have distinctive fatty acyl unsaturation profiles. In the livers of Rana pipiens, Xenopus laevis and Rana catesbeiana tadpoles the major unsaturated fatty acyls are monoenes and the polyene acyls of the dietarily essential fatty acids $18: 2 \omega 6$ and $18: 3 \omega 3 .{ }^{238,241}$ Frog livers contain less monoenes and more PUFA products of $\Delta 6$ - and $\Delta 5$-desaturations of the essential fatty acids. Heart lipids from $R$. pipiens tadpoles or frogs have higher proportions of biodesaturated polyene acyls than do their respective liver lipids. Liver lipids of $\boldsymbol{R}$. catesbeiana tadpoles contain $67 \%$ neutral lipids, frog liver lipids contain $75 \%$ polar lipids. Both neutral and polar lipids from the tadpoles are higher in monoenes than are the corresponding lipids from frogs. We do not know the relative amounts or the fatty acyl compositions of the PL subclasses. Tadpole livers metabolize intraperitoneally injected $\left[1-{ }^{14} \mathrm{C}\right] 18: 2 \omega 6$ unexpectedly vigorously, considering how few derivatives accumulate in their lipids. In $18 \mathrm{hr}, 20 \%$ of the counts are incorporated into liver lipids, of which $40 \%$ is in polar lipids. Frogs incorporate $10 \%$ of the carbon counts into liver lipids, of which $90 \%$ is in polar lipids. Of the total counts in either tadpoles or frogs, $37 \%$ is in biosynthesized $\omega 6$-PUFA derivatives. This degree of metabolism may be compared with that in livers of rats $6 \mathrm{hr}$ after receiving labeled 18:2 (Table 2); normal rats incorporate 3.5\% of injected label and convert $25 \%$ of it to the $20: 4$ derivative; hypothyroids incorporate $0.9 \%$ of label and convert only $8.7 \%$ to $20: 4$.

Thyroid hormone specifically induces Anuran metamorphosis. ${ }^{198}$ During the natural development of tadpoles, the proportions of liver lipid mono-unsaturated acyls decrease and those of polyene acyls increase at the stages when plasma thyroxine levels rise ${ }^{588}$ and urea synthesis begins, ${ }^{16}$ as shown in Fig. 3 . The changes in unsaturation pattern follow the natural surge of circulating [T4] at least as closely as does the appearance of urea. Thyroid-induced metamorphosis is accompanied by similar lipid alterations. Immersing tadpoles in $1 \mu \mathrm{M} \mathrm{T} 3$ for $48 \mathrm{hr}$ converts their liver lipid profile to or beyond the polyene pattern typical of frogs. Injecting tadpoles once with $\mathrm{T} 3,0.2 \mu \mathrm{g} / \mathrm{g}$, produces similar but lesser and transient changes over 5 days. Injecting frogs with $\mathrm{T} 3,1 \mu \mathrm{g} / \mathrm{g}$, decreases liver monoene contents transiently over 2 days and in addition partially depletes $\omega 6$-PUFA-as it does more rapidly in hypothyroid rat liver organelle membranes (Table 1). When tadpoles are injected with $\mathrm{T} 3$ at time $=0$ and with labeled $18: 2$ at $18 \mathrm{hr}$, the following changes occur. The ratio of liver PL to total lipids increases to reach $76 \%$ by the 8 th day after $\mathrm{T} 3$ injection. The PL incorporate more of the ${ }^{14} \mathrm{C}$, the neutral lipids, less. The proportion of label in desaturation products of 18:2 in the PL increases linearly over the 8 days to become 2.5 times greater than in untreated tadpoles or in frogs. Similar T3 treatment changes tadpole liver endoplasmic reticulum from simple vesicular membranes to more complex lamellar structures. ${ }^{597}$

We conclude that, in tadpole livers, fatty acyl unsaturation patterns reflect a high content of neutral lipids that are repleted with monoene acyl groups. In frogs, unsaturation 


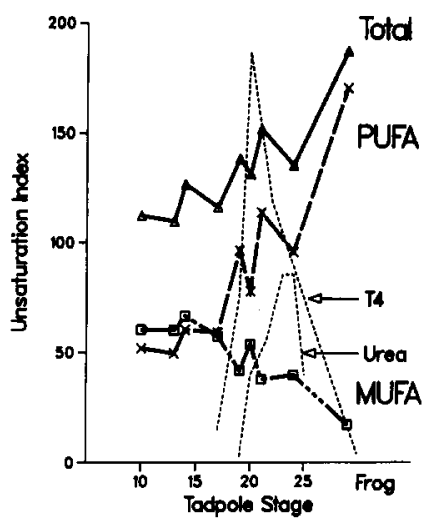

Fig. 3. Developmental changes in the overall unsaturation index and in the contributions of polyunsaturated and mono-unsaturated fatty acyls in the total liver lipids of livers of $\boldsymbol{R}$. catesbeiana tadpoles; and comparisons with values in frogs. Untreated tadpoles were observed from Stage 10 to Stage 24. Values of unsaturation indices calculated from measured fatty acyl compositions are shown for the sum of all fatty acyls (Total), for the sum of the polyunsaturated fatty acyls (PUFA) and for the sum of the mono-unsaturated fatty acyls (MUFA). The dashed lines indicate values for plasma thyroxine concentrations (T4, in $\mathbf{n g} / 40 \mathrm{ml}$ ) taken from Ref. 588 and for hepatic urea concentrations (Urea, in $\mu \mathrm{g} / 0.4 \mathrm{~g}$ ) taken from Ref. 16.

comprises both the high polyene content characteristic of their PL, and a greater proportion of PL in their liver lipids. Both natural and T3-induced metamorphosis involve a shift from the tadpole lipid pattern toward the frog pattern. The shift is early enough to serve as a biochemical marker for metamorphosis, like urea synthesis. Tadpole livers convert 18:2 to $\omega 6$-PUFA derivatives as well as frog livers do, so thyroid hormone is not necessary for the desaturations involved, but perhaps is necessary for the synthesis of PL that retain the PUFA produced. T3 injection also increases desaturations in tadpoles, indicating that T3 is sufficient but not necessary to stimulate Anuran hepatic $\Delta 6-$ and $\Delta 5$-desaturases.

\section{J. Fatty Acid Oxidation}

Fatty acid oxidation rate is proportional to the thyroid state. ${ }^{1}$ Mechanisms that regulate the partition of fatty acids between oxidation and esterification in liver are discussed by Zammit. ${ }^{650}$ The major site of control of fatty acid oxidation itself is in the mechanisms that transport cytoplasmic fatty acyl-CoA, with the acylcarnitine as an intermediate, to the fatty acyl-CoA dehydrogenase in the mitochondrial matrix adjacent to the electrontransport chain. Cytoplasmic acyl-CoA $\rightarrow$ acylcarnitine conversion is 'rate-limiting' for long-chain fatty acid oxidation in isolated mitochondria, ${ }^{159,392}$ because the transferase system in intact mitochondria is partly latent. Thyroid hormones increase hepatic oxidation of $18: 1$ by accelerating entry of $18: 1-\mathrm{CoA}$ into the matrix. ${ }^{411}$

Carnitine acyltransferases, as exemplified by the carnitine palmitoyltransferase (CPT), are the most important, although the translocase may also regulate. The CPT enzyme(s) are highly regulated ${ }^{36,54}$ by positive and negative effectors; by covalent modification (phosphorylation activates ${ }^{210}$ ); by substrate and product; $;^{55.461-463}$ and by vicinal phospholipids in the inner or outer layers of the mitochondrial inner membrane. ${ }^{461,462,643,650,653}$ Kinetic measurements are a dubious basis for determining mechanisms of thyroid control over CPT, because the amphipathic substrates and products form micelles readily and may act non-specifically as detergents. ${ }^{89}$

The outer enzyme, $\mathrm{CPT}_{0}$, in intact liver mitochondria normally has about $10-20 \%$ of the total CPT activity found in detergent-lysed mitochondria, depending on the detergent (Refs 32, 53; see Table 4); CPT activity in the intact membrane has been estimated as $0.2 \%$ of inner enzyme, $\mathrm{CPT}_{i}$, activity ${ }^{32} \mathrm{CPT}_{\mathrm{i}}$ activity is expressed fully by gentle disruption of the membranes, but loses appreciable activity when solubilized by more drastic extractions, suggesting that its active configuration requires lipid. 
TABLE 4. The Effects of Thyroid State (Fed Rats) on 16:0 Oxidation $\left(\mathrm{nmol} / \mathrm{min} / \mathrm{mg}\right.$ protein; $37^{\circ} \mathrm{C}$ ) in Isolated Hepatocytes; and on CPT and Total CPT Activity, and by Difference, CPT $_{\mathrm{i}}$ (nmol 16:0-Carnitine Formed/min/mg protein; $30^{\circ} \mathrm{C}$ ) in Liver Mitochondria; from Refs 571,572

\begin{tabular}{llrrr}
\hline System & Activity & Normal & Hypothyroid & Hypo + LT3 \\
\hline Hepatocytes & Oxidation & 1.5 & 0.3 & 2.6 \\
Mitochondria & CPT & 3.8 & 1.7 & 6.7 \\
& Total CPT & 33.8 & 29.6 & 31.5 \\
& $\Delta=$ CPT $_{i}$ & 30.0 & 27.9 & 24.8 \\
\hline
\end{tabular}

Whether $\mathrm{CPT}_{0}$ and $\mathrm{CPT}_{\mathrm{i}}$ are two proteins with different primary structures and lipid affinities, or one protein in the different lipid environments of the inner and outer layers of the mitochondrial inner membrane, is crucial for determining thyroid control mechanisms. CPT $\mathrm{C}_{0}$ and $\mathrm{CPT}_{i}$ are said to be the same enzyme by the criteria of their physical properties (molecular weights, solubilities, electro-mobilities), and kinetics (e.g. $K_{\mathrm{m}}$ values of membrane-free enzymes for their several substrates), ${ }^{33,128}$ and their immunologic identity. ${ }^{32,50}$ Evidence presented for liver mitochondrial $\mathrm{CPT}_{0}$ and $\mathrm{CPT}_{\mathrm{i}}$ being different enzymes, or more ambiguously, different 'forms of CPT', includes the following. $\mathrm{CPT}_{0}$ and $\mathrm{CPT}_{\mathrm{i}}$ have different susceptibilities to inhibition by malonyl-CoA, thought by some to reside in a regulatory subunit; ${ }^{651}$ and different $M_{\mathrm{r}}$ values $\left(\mathrm{CPT}_{\mathrm{o}}=63 \mathrm{kDa}\right.$, $\left.\mathrm{CPT}_{\mathrm{i}}=67 \mathrm{kDa}\right) .^{491}$ Polyclonal antibodies raised against a mono-disperse, PL-free CPT。 prepared from beef liver mitochondria uncontaminated with peroxisomes ${ }^{490,491}$ (which contain molecularly distinct short- and medium-chain acyltransferases ${ }^{35}$ ) and against a purified $\mathrm{CPT}_{i}$, do not cross-react by Western blot or by inhibiting activity. ${ }^{491}$ Although these experiments are thought to demonstrate that $\mathrm{CPT}_{0}$ and $\mathrm{CPT}_{i}$ differ in physical properties rather than in membrane environment, in earlier work antibodies against a purified homogeneous $\mathrm{CPT}_{0}$ purified from calf liver mitochondria ${ }^{313}$ inhibited $\mathrm{CPT}_{0}$, but those from 1 out of 3 rabbits also inhibited $\mathrm{CPT}_{\mathrm{i}}{ }^{65,314}$ Taken together with observations that urea or guanidine convert CPT $\rightarrow \mathrm{CPT}_{i}$, Kopec and Fritz ${ }^{314}$ thought CPT $\mathrm{C}_{i}$ is a

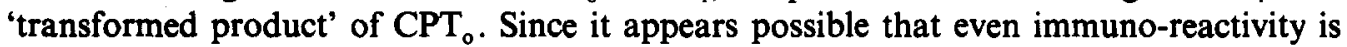
modified by membrane environment, yet more specific molecular properties such as primary structure may be needed to determine when gene expression of $\mathrm{CPT}_{i}$ or CPT $_{o}$, and/or membrane lipid changes, regulate CPT activities.

Hypothyroidism halves the activity of the $\mathrm{CPT}_{0}$ in isolated rat liver mitochondria, and reduces ${ }^{14} \mathrm{CO}_{2}$ production from ${ }^{14} \mathrm{C}$-palmitoyl-CoA in isolated hepatocytes by $80 \%$ (Table 4). T3-treatment restores $\mathrm{CPT}_{0}$ activity and 16:0 oxidation even beyond normal levels (?thyrotoxic). Total CPT activity, in lysed mitochondria, remains similarly high in all three groups; by difference, $\mathrm{CPT}_{\mathrm{i}}$ in hypothyroids is at about the control activity, but in T3-treated animals is rather lower. The approximate constancy of the total CPT activity speaks for there being one enzyme that thyroid treatment converts from inactive $\mathrm{CPT}_{\mathrm{i}}$ to active $\mathrm{CPT}_{\mathrm{o}}$ form, thereby increasing the oxidation of 16:0. Given the uncertainties of these activity measurements, additional quantitation of thyroid effects on amounts of each 'form' are needed, as well as more direct determination as to whether or not thyroid induces only the CPT form.

It is also possible that thyroid state regulates $\mathrm{CPT}_{\mathrm{o}}$ activity in the intact cell indirectly, through [malonyl-CoA] and CPT sensitivity to inhibition by malonyl-CoA. In perfused livers, hypothyroidism decreases [malonyl-CoA] and hyperthyroidism does not affect [malonyl-CoA], so Müller et al ${ }^{411}$ doubt its regulatory role in thyroid effects. However, in freeze-clamped livers, hypothyroidism doubles [malonyl-CoA] while hyperthyroidism decreases it slightly, ${ }^{365}$ effects consistent with a regulatory role. Malonyl-CoA inhibits the increased activity of CPT ${ }_{0}$ that follows T3-treatment (Table 4) less than normally, ${ }^{55}$ accenting the decreased concentration. The diminution of malonyl-CoA inhibition of fatty acid oxidation is thought to permit a futile, calorigenic cycle of simultaneously accelerated synthesis and oxidation. The CPT in hypothyroid rats (Table 4) retains normal sensitivity to the high [malonyl-CoA], and so is depressed. 
When liver mitochondria from normal rats are preincubated in $\mathrm{KCl}$-media, malonylCoA does not inhibit; mitochondria from hypothyroid rats lose sensitivity more slowly and those from hyperthyroid rats, faster. Therefore, CPTo is thought to be regulated by components of membranes that respond to physiological stimuli ${ }^{31,461,651,653}$ Malonyl-CoA acts on membrane-bound CPT ${ }_{0}$ like a classical negative allosteric effector, ${ }^{36}$ but does not inhibit after the membrane is exposed to phospholipase $\mathrm{C}$ (which itself activates $\mathrm{CPT}_{0}{ }^{643}$ ), or after CPT is solubilized. Malonyl-CoA inhibition of CPT $_{0}$ depends on high membrane fluidity: in liver mitochondria (especially from fed rats) kept at $25-37^{\circ} \mathrm{C}$ (but not at $<25^{\circ} \mathrm{C}$ ), slow conformation changes make $\mathrm{CPT}_{0}$ less sensitive to malonyl-CoA and increase CPT activity. ${ }^{651}$

Another mechanism may involve regulation of $\mathrm{CPT}_{0}$ through phosphorylation status. Thyroid state modifies the effects of starvation. Fasting increases oxidation $25-50 \%$ in mitochondria from euthyroid rats, $300-400 \%$ in hypothyroids, and not at all in thyrotoxics (where it is already increased). Starvation normally augments both CPT ${ }_{0}$ and (acyl)carnitine translocase activities, and hepatic fatty acid oxidation; cycloheximide blocks the increase in $\mathrm{CPT}_{\circ}$ activity, indicating that starvation induces microsomal synthesis of new protein(s) ${ }^{519}$ The new protein might be either $\mathrm{CPT}_{0}$ or a protein phosphatase or kinase, since starvation signals protein phosphorylation, and phosphorylation may activate CPT. ${ }^{210}$

(Acyl)carnitine translocase mediates a 1:1 exchange of carnitine and an acylcarnitine. At $37^{\circ} \mathrm{C}$, the exchange rate appears to be much greater than the capacity for $\beta$-oxidation. However, at low tissue [acylcarnitine], the translocase may come to share control over oxidation with the CPT ${ }_{0}{ }^{55}$ Urinary excretion of carnitine, and serum carnitine concentration, are proportional to serum [T4] in hypo- and hyperthyroid subjects, ${ }^{375}$ but there are no measurements that show cell carnitine availability limits either the translocase or CPT activity. It is not known if thyroid state affects translocase activity, but thyroid regulation of mitochondrial inner membrane $\mathrm{CL}$ content and composition offers a possible mechanism to explain some effects attributed to membrane environment. The purified translocase contains bound $\mathrm{CL},{ }^{149}$ and reconstitution of the solubilized (acyl)carnitine translocase requires cardiolipins. ${ }^{443}$ Adriamycin, which binds CL specifically (see Section IV.B.5), inhibits both native and reconstituted translocase activity. ${ }^{410}$ Since the purified CPT is also activated by CL, Noël and $P a n d e^{443}$ propose that $C L$ provides orienting binding sites for the translocase and the transferase, and thereby facilitates the flow of acylcarnitines from the outer to the inner layers of the membrane.

The asymmetry of $\mathrm{CL}$ distribution, $75-90 \%$ being in the inner layer of the inner membrane of mitochondria (see Ref. 106), resembles that of the activities of liver CPT and $\mathrm{CPT}_{\mathrm{i}}$ (Table 4). If CL does bind CPT firmly in vivo, and if the distinction between $\mathrm{CPT}_{\mathrm{o}}$ and $\mathrm{CPT}_{\mathrm{i}}$ reflects only their membrane environment, the paucity of $\mathrm{CL}$ in the outer face may account for the relatively small fraction of CPT in the outer face. Although effects of thyroid state on the sidedness of $\mathrm{CL}$ in hepatic mitochondrial inner membranes have not been reported, hypothyroidism increases the proportion of liver mitochondrial CL/ $/$ PL by $72 \%$ while maintaining the high $18: 2$ content of the CL. ${ }^{248}$ The physical properties of CL favor the excess CL being in the inner layer (see Section IV); less transferase would then exist in the CPT form (Table 4). Thyrotoxicosis also increases liver mitochondrial CL/PL $34 \%$, but the CL is depleted in $18: 2$ by $63 \% .^{511}$ If this abnormal $\mathrm{CL}$ does not bind CPT fully, the fraction in the $\mathrm{CPT}_{0}$ form would increase.

In liver mitochondria of $1-5$ day old pigs, $\mathrm{CPT}_{\mathrm{o}}=\mathrm{CPT}_{\mathrm{i}} \cdot{ }^{37}$ In liver mitochondria of neonatal rats none of the CPT is in latent form, ${ }^{17} \mathrm{CL} / \mathrm{PL}$ is low, and the CL 18:2 content is halved, as compared with adults. In adult heart mitochondria, LT3 $(0.33 \mu \mathrm{g} / \mathrm{g} / \mathrm{day} \times 2$ days) given to hypothyroid rats does not change the activity of $\mathrm{CPT}_{0}$, although it increases

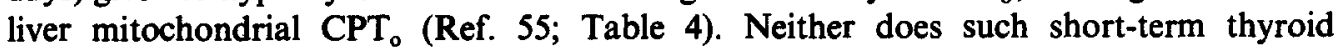
hormone treatment of normal rats (LT3, $1 \mu \mathrm{g} / \mathrm{g} / \mathrm{day} \times 3$ days) change heart mitochondrial 18:2 content or CL/PL ratio; ${ }^{237}$ we did not examine CL/PL values in heart mitochondria of T3-treated hypothyroid rats. The apparent disparity in T3 effects on heart and liver may reflect only that heart responses require greater dose-time schedules, as is explicated in 
Section IV.D. Thyroid feeding for 6 days accelerates palmitate oxidation in perfused hearts of dogs; ${ }^{174}$ and LT4-injection, $\sim 0.25 \mu \mathrm{g} / \mathrm{g} /$ day $\times 10$ days, more than doubles CPT activity in guinea pig heart homogenates. ${ }^{58}$ The latter workers attribute the increase in fatty acid oxidation to the high plasma [FFA], and the doubled heart [acylcarnitine] and [total carnitine], after thyroid pretreatment.

In mitochondria of mature brain, the activity of $\mathrm{CPT}_{\mathrm{o}}$ plus $\mathrm{CPT}_{\mathrm{i}}$ is about $25 \%$ of that in liver; $\beta$-oxidation is much slower, and neither $\mathrm{CPT}_{\mathrm{o}}$ or $\mathrm{CPT}_{\mathrm{i}}$ is rate-limiting. ${ }^{41}$ Neither thyroid-treatment nor starvation increases activities in these low- $\mathrm{CL}^{632}$ mitochondria. The higher $\mathrm{CPT}_{\mathrm{o}}$ activity in fetal brain decreases $50 \%$ after weaning, as what small amounts of $C L$ there are in the adult brain begin to appear (see Section IV.D).

\section{K. Prostanoid Synthesis}

Brief treatment of rats with $\mathrm{LT} 4,0.2 \mu \mathrm{g} / \mathrm{g} /$ day $\times 2$ days, increases prostaglandin synthetase activity by $179 \%$ in kidney and $300 \%$ in lung; at the same time, it inhibits renal $\mathrm{PGF}_{2 \alpha}$ metabolism by $40 \%$ in pathways that include conversions by 15 -hydroxyprostaglandin dehydrogenase and prostaglandin $\Delta 13$-reductase, and prostaglandin 9-hydroxydehydrogenase. ${ }^{255,406}$ The prostaglandin synthetase of spleen microsomes, as measured by the conversion $20: 4 \rightarrow \mathrm{PGE}_{2}$, also increases in hyperthyroidism but is unaffected by hypothyroidism; however, concentrations of indomethacin or phenylbutazone that inhibit $\mathrm{PGE}_{2}$ formation about $50 \%$ in preparations from normal and hyperthyroid animals inhibit only about $20 \%$ in hypothyroids. ${ }^{155}$ It seems unlikely that $\mathrm{T} 3$ stimulates conversion of enough hepatic microsomal 20:4 acyl groups to prostaglandins to contribute to the observed rapid depletion of 20:4 (see Section II.A).

\section{Sulfolipid Synthesis}

Brain myelination and maturation require adequate thyroid hormone levels. ${ }^{446}$ Hypothyroidism in the neonate retards accumulation of brain cholesterol, cerebrosides, and sulfatides. T3-treatment at 18 days after birth does not replenish the very low amounts of these lipids, although T3 given much earlier does stimulate sulfatide synthesis. ${ }^{625}$ The defect in sulfatide synthesis is caused by depressed synthesis of $3^{\prime}$-phosphoadenosine- $5^{\prime}$ phosphosulfate and/or the subsequent NADPH-dependent (and thereby thyroid-dependent?; see Section II.C) reduction of the sulfate of this donor to $\mathrm{SO}_{3}^{2-}$. Thyroid effects have been studied in myelin-synthesizing glial cells grown in culture, free of contamination with neurones (which complicate interpretations by producing nerve growth factors in response to T3) ${ }^{315}$ Growth in T3-supplemented media for 3-5 days stimulates $\left[{ }^{35}\right.$ S]sulfate incorporation into sulfatides and sulfogalactosyl dialkylglycerols, as well as synthesis of fatty acids and cholesterol, and activity of $2^{\prime}, 3^{\prime}$-cyclic-nucleotide $3^{\prime}$-phosphodiesterase. These cultures progressively lose their sensitivity to T3, rather like neonatal rats lose brain responses to developmental thyroid effects (see Section IV.D).

\section{Summary}

The pervasiveness of thyroid-state influence on enzymatic steps in lipid metabolism is striking. Especially in the branching pathways of fatty acid and cholesterol metabolism (Fig. 1) and conversions of glycerol-3-phosphate to triglycerides and phosphoglycerides (Fig. 2), as well as in fatty acid desaturations, almost all steps are thyroid-sensitive. Several points can be made: (i) the conversions of carbohydrate to lipids show an analogous prevalence of steps sensitive to insulin, and to protein phosphorylation. ${ }^{212}$ Observed effects of thyroid hormones on protein phosphorylations are suggestive, but no such rigorous correlation has been made with the enzymes involved in lipid conversions. (ii) Thyroid levels do not exert their influence at a single 'rate-controlling' enzyme in a lipid pathway, but on successive enzymes. To regulate the evolution of the lipid product, the hormone must act differentially on each step, and several steps may contribute to rate-control. Such 
analysis of thyroid controls has been done on the steps of mitochondrial oxidative phosphorylation (see Section IV). (iii) Thyroid stimulation of gene expression can account for only a few of the many sensitive loci in lipid metabolism. In the succeeding sections, mechanisms are discussed whereby thyroid-effected changes in lipid metabolism may amplify signals of T3-receptor occupancy to modulate membrane-dependent catalysts, transporters, and receptors.

\section{CELL MEMBRANE AND ASSOCIATED SYSTEMS}

Plasma membranes contain a number of enzymes, transporters and receptors that are thyroid-responsive. Such sensitivity may be mediated by mechanisms of gene expression, covalent modifications of the proteins, and lipid-mediated membrane-dependency of the proteins, and ion leaks.

\section{A. Lipid Composition and Physical Properties}

All the thyroid-sensitive active proteins in the plasma membrane are known to change activity when membrane lipid composition is manipulated. However, I find no reports on fatty acyl and cholesterol compositions of purified plasma membranes from animals in different thyroid states, so causal connections with altered activities remain uncertain. Inferences from coincidence and circumstantial evidence should be guarded, especially because the plasma membrane differs in several important respects from organelle membranes. Lipid composition of purified hepatocyte plasma membranes (and erythrocyte membranes) is quite different from that of the thyroid-responsive membranes of the endoplasmic reticulum, mitochondria, and nuclei (plasma membranes are usually sedimented with nuclei as heavy fractions). The unsaturation index of the $\Sigma$ PL of hepatocyte plasma membrane ranges from $70^{301,581}$ to $130,{ }^{423}$ as compared with about 190 in the organelle membranes, and 215 in heart sarcolemma. ${ }^{2}$ Twenty percent of the liver plasma membrane and erythrocyte membrane PL are sphingomyelins, with their characteristically high contents of saturated long chain fatty acyls, and UI $\sim 45$, which lowers overall unsaturation. Molar ratios of free cholesterol/PL contents in plasma membranes are $0.35-0.6$, and in mitochondria and rough endoplasmic reticulum, $0.03-0.05 .132,565,581,615$ Some investigators take the presence of cholesterol in mitochondria to denote contamination with heavy fractions, but Rogers et al. ${ }^{498}$ find that cholesterol-feeding increases mitochondrial cholesterol content in rat liver (but not in other tissues), depresses State 3 respiration, and desensitizes the ATP-synthetase toward oligomycin inhibition.

An acute lipogenic stimulus-feeding carbohydrate for a few hours to a starved rat-does not deplete hepatocyte plasma membrane 18:2 acyls and actually increases 20:4 acyl contents (in organelle membranes, it extensively replaces both 18:2 and 20:4 with newly synthesized MUFA and saturated fatty acyls), but decreases the cholesterol/PL ratio by $-24 \% .{ }^{581}$ More prolonged EFA-deficiency, in Ehrich cells grown in EFA-deficient mice, does severely deplete plasma membrane $\omega$-6-PUFA $(18: 2$ by $-80 \%, 20: 4$ by $-45 \%)$ and replaces them with biosynthesized $\omega 9$-PUFA $(18: 1,20: 3,22: 3)$, but the cholesterol/PL ratio and the proportions of PL classes remain at control levels. ${ }^{308.581}$ Thyroid effects on plasma membrane lipid contents need measuring in preparations certified to be minimally contaminated with organelle membranes-it seems possible that plasma membrane and organelle membrane fatty acyl compositions do not respond similarly to thyroid state.

However, thyroid state or dietary lipids affect plasma membrane general physical properties, and enzyme and transporter activities, in a manner consistent with mediation through lipid changes. Plasma membrane lipids are known to modify the mechanism of a number of receptors. ${ }^{361}$ Arrhenius plots of the motion parameter of 12-nitroxide stearic acid probes in isolated plasma membranes of Ehrlich tumor cells exhibit transitions at $31^{\circ} \mathrm{C}$ and $19^{\circ} \mathrm{C}$ when prepared from control-fed mice, and at $31.5^{\circ} \mathrm{C}$ and $24.5^{\circ} \mathrm{C}$ when prepared from EFA-deficient animals. ${ }^{308}$ The order parameter at $37^{\circ} \mathrm{C}$ is greater in the EFA-deficient 
membranes. These changes are attributed to the decrease in unsaturation at the $\omega 6$-position, where larger kinks in the fatty acyl chains disturb the packing order especially toward the center of the bilayer. In contrast, when fatty acyls of the plasma membranes of murine $T$ lymphocyte tumor cells in culture are substituted by less or more unsaturated fatty acyls, the temperature-dependence of order parameters and rotational correlation times of both 5- and 12-nitroxide probes remain normal (although enzyme activities change; see below). ${ }^{481}$ It should be noted that the UI of the Ehrlich tumor cells in animals on normal diets is $140-170$, higher than the UI of liver plasma membranes, and that tumor cell lipid metabolism and membrane composition differ from those of normal cells. ${ }^{517}$ In thyrotoxic rats, liver plasma membrane preparations are abnormally rigid and ordered (as estimated from polarization of fluorescent cis-and trans-parinaric acid probes), and lose their reactivity to added insulin or glucagon. ${ }^{534}$ Plasma membranes from hypothyroid rats are normally ordered, but $\mathrm{T} 3$ added in vitro increases their rigidity. Arrhenius plots of fluorescence polarization of these probes in plasma membranes from thyrotoxic rats inflect at $25^{\circ} \mathrm{C}$, in hypothyroids, near $18^{\circ} \mathrm{C}$. Such abnormalities seem consistent with excess thyroid levels decreasing fatty acyl unsaturation, and emphasize the need for lipid analysis since thyroid-treatment usually increases unsaturation in organelle membranes.

\section{B. cAMP Synthesis and Hydrolysis}

The concentration of cAMP in contact with its target enzymes mediates thyroid state regulation in several cases, notably the hormone-sensitive lipase. Hypothyroidism decreases the rise of [cAMP] in adipocytes after application of lipolytic hormones (catecholamines, glucagon, ACTH). ${ }^{97,98,616}$ The mechanisms include simultaneous impairment of synthesis and enhancement of degradation of cAMP, decreased cAMP-dependent protein kinase activity, and decreased phosphorylation of the lipase that lowers its activity. Hyperthyroidism increases adipocyte [cAMP] rise effected by lipolytic agents, and thereby increases lipolysis. Whatever the mechanisms, thyroid control over [cAMP] appears to be analogous to that over hepatic [cholesterol] in that the hormone differentially regulates their synthesis and (more acutely) their degradation, and both cAMP and cholesterol are effectors in a variety of enzyme and transporter processes.

\section{Adenylate Cyclase}

Thyroid hormone regulation of the adenylate cyclase system in plasma membranes is important in lipid metabolism because cAMP mediates reversible phosphorylation of many enzymes in these metabolic pathways, and because thyroid state regulates the adenylate cyclase system at several membrane-dependent components that respond to lipid changes. Many regulators, together with the thyroid state, are tissue-specific in controlling the multicomponent adenylate cyclase system. Some thyroid effects are primary in the sense that they occur during in vitro incubation with plasma membranes. Some are exerted secondarily and result in permissive effects of thyroid state on such regulators of lipid metabolism as catecholamines, ${ }^{579}$ glucagon and insulin.

Some thyroid effects may be mediated by plasma membrane PL. Adenylate cyclase activity and its coupling to hormone receptors require plasma membrane PL. Removal of acidic PL (PI, PS) but not neutral PL (PC, PE, Sph) by phospholipase C inactivates the cyclase and ablates its activation by epinephrine or glucagon. ${ }^{349-351,497,509}$ Addition of PS (and to a lesser extent PE or Sph, but not PC or $\mathrm{CL}$ ) reconstitutes the solubilized, inactive cat heart cyclase. ${ }^{351}$ In rat liver plasma membranes, EFA-deficiency depresses glucagonstimulated adenylate cyclase activity, ${ }^{363}$ but in titrations with $\Delta$ [dietary linoleate], contents of $\omega 6$-PUFA in phosphatidylcholines correlate inversely with activity, ${ }^{423}$ it is not clear to what degree fatty acyl dependency of the adenylate cyclase or the glucagon-receptor systems is being measured. Repletion of the plasma membrane phospholipids of a T-lymphocyte tumor cell or LM cell line with saturated fatty acyls suppresses basal and 
PGE $_{2}$-sensitive adenylate cyclase (and $\mathrm{Na}^{+} \mathbf{K}^{+}$-ATPase, see Section III.D) activity, while repletion with 18:1 or 18:2 fatty acyls enhances activity, both compared with cells grown with minimal amounts of $18: 2 .{ }^{133,481}$ The constancy of overall membrane lipid fluidity parameters during these substitutions suggests a more specific interaction between the PL fatty acyls and the enzyme system. In contrast to the thymocyte and fibroblast cultures, when plasma membrane fluidity of turkey erythrocytes is progressively increased by

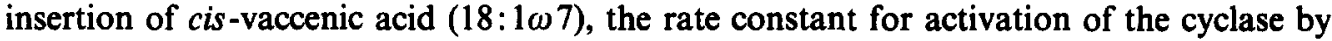
epinephrine increases linearly with membrane fluidity (measured by fluorescence polarization of a probe), indicating that coupling between the epinephrine receptor and the cyclase depends on the lipids. ${ }^{208,494}$ The maximal activity of the catalytic unit itself, when stimulated by several activators, also increases with lipid fluidity. In contrast, adenosine activates the cyclase in these erythrocytes independently of membrane fluidity, probably at a site on a G-protein.

Either hyper- or hypothyroidism in vivo or incubation with hormone in vitro can modulate adenylate cyclase activity in broken-cell preparations. ${ }^{538}$ Adenylate cyclase activity is twice-normal in minces of fat pads from hyperthyroid rats, and half-normal in hypothyroids. ${ }^{322}$ Epinephrine stimulates lipolysis, when added to adipose tissue of hyperthyroid rats, to maximal levels $2-3$ times those reached in tissue from euthyroid rats. Even high [epinephrine] fails to activate the cyclase in fat cells from hypothyroid rats; T4-treatment restores the response in $48 \mathrm{hr}$.

In vivo mechanisms that mediate such thyroid effects in adipose and other tissues include changes in: numbers and affinity of $\beta$-adrenergic receptors in plasma membranes; coupling between receptors and cyclase components; and function and amounts of regulatory and catalytic cyclase components. ${ }^{579}$ In vivo thyroid effects differ in heart, adipose tissue, lung, renal medulla, and nucleated erythrocytes from those in liver. ${ }^{377}$ Hypothyroidism decreases, and hyperthyroidism increases, the number of $\beta$-adrenergic receptors in heart, ${ }^{609}$ fat, ${ }^{377}$ turkey erythrocytes, and rat reticulocytes. In hyperthyroid animals, the cardiac receptors have abnormally high affinity for $\beta$-adrenergic agonists. Since blood and tissue [catecholamine] are in the normal range in thyrotoxicosis, the many cardiovascular signs seem to arise from hypersensitivity to catecholamines. However, see Ref. 349 for the opposing view that the thyrotoxic hearts of several species are not hypersensitive to norepinephrine, based mainly on their mechanical function.

The rat liver shows effects opposite to those in the tissues just discussed. Perhaps this specificity is accounted for by observations that, in normal rats, $\alpha$-adrenoceptors mediate activation of hepatic glycogen phosphorylase by catecholamines, in other species, $\beta$-receptors (see Ref. 485). Hypothyroidism increases total cyclase activity in rat hepatic plasma membranes by $30-45 \%,{ }^{200,284}$ and the number of $\beta$-adrenergic receptors by $2-$ to 3-fold. ${ }^{579}$ Hyperthyroidism decreases the number of liver $\beta$-adrenoceptors, cyclase activity, and response of cyclase to $\beta$-agonists, ${ }^{579}$ or does not change cyclase activity and the $\beta$-receptor response. ${ }^{284}$ The cyclase deficiencies in hyperthyroidism are not associated with a decrease in amount of G-protein, but with lesser effectiveness of the G-proteins in reconstituting adenylate cyclase in other membranes, and/or with decreases in function of the catalytic cyclase unit (see Ref. 579 for additional references).

In vivo effects on the permanent coupling between $\beta$-adrenergic receptors and the catalytic subunit of adenylate cyclase through the regulatory G-proteins are also tissuespecific. Hypothyroidism decreases the amount of G-protein in rat reticulocyte plasma membranes together with the number of $\beta$-receptors; both defects are tentatively ascribed to decreased protein synthesis. ${ }^{579}$ Some of the effects of hypothyroidism on the adipocyte adenylate cyclase system are apparently mediated by the GTP-dependent regulatory proteins. ${ }^{378}$ The (measured) amount and the activity of the inhibitory protein $G_{i}$ increases while the amount of the stimulatory protein $G_{s}$ is little changed. Thereby the inhibitory regulation by GTP, adenosine (which inhibits in fat cells), and other metabolites is enhanced by up to 50 times. At the same time, cyclase activity and response to activators are dampened, probably through a change in a $\beta$ - or $\gamma$-subunit common to $G_{i}$ and $G_{s}$. It is unusual for the hypothyroid state to evoke an increase in the amount of a protein, 
because hypothyroidism diminishes gene expression; the mRNAs for the subunit peptides will be interesting to see.

Thyroid state not only modifies $\beta$-receptor-mediated lipolytic and positive cardiac chronotropic and inotropic effects, but also what appear to be $\alpha$-receptor-mediated effects on these parameters. Thus, norepinephrine (a mixed $\alpha-\beta$ agonist) elicits no lipolytic response in adipocytes from hypothyroid humans although those from normals are quite responsive; addition of an $\alpha$-receptor antagonist normalizes the lipolytic action, and hypothyroidism augments an $\alpha$-response which inhibits the adenylate cyclase. ${ }^{191,192,502,503}$ Chronotropic dose-effects of norepinephrine are subnormal in isolated atria of hearts from thyrotoxic rats but normal in those from hypothyroid animals, while dose-effects of epinephrine decrease in hypothyroids and remain normal in thyrotoxics. ${ }^{583}$ As measured by inotropic actions, atria from hypothyroids have suprasensitive $\alpha$-receptors while their $\beta$-receptors are 10-times less sensitive than normals, from measured potency ratios of agonists, and the new susceptibility to inhibition by an $\alpha$-receptor blocking agent. ${ }^{30,327-329}$ The sensitivities revert to or beyond control levels after hypothyroid animals receive 7 daily T4- or T3-injections, the only dose schedule examined. Alpha-agonists also make aortae from hypothyroid rabbits contract more forcibly than normals. ${ }^{192}$ Hypothyroidism also augments the $\alpha$-adrenoceptor-mediated rise in [cAMP] in electrically driven contracting rat heart muscle, and reciprocally depresses the $\beta$-receptor-mediated rise; T4-treatment of hypothyroid rats restores the normal pattern in 8 days. ${ }^{326}$ As in measurements of total cyclase activity and number of adrenergic receptors, liver cells show the opposite pattern: in hepatocytes from hypothyroid rats $\alpha$-receptor mediation falls and $\beta$-receptor mediation increases reciprocally. ${ }^{485}$

Evidence is presented that the reciprocity of $\alpha$ and $\beta$ effects reflects interconversion of myocardial $\alpha$ - and $\beta$-adrenoceptors through an allosteric change in one common precursor adrenoceptor. ${ }^{327-329}$ The $\beta$-receptors convert to $\alpha$-receptors when the temperature of isolated hearts of frogs or rats is lowered to $<17^{\circ} \mathrm{C} .{ }^{329}$ Others ${ }^{70}$ confirm that a $\beta \rightarrow \alpha$ transition occurs on cooling isolated frog hearts, but from the effects of irreversible antagonists conclude that separate pools of $\beta$ - and $\alpha$-precursor receptors are recruited at the different temperatures. Since these studies on isolated hearts seem to preclude adaptations through hormone actions, it seems likely that temperature-sensitive plasma membrane fluidity is involved, since either cold or hypothyroidism decreases fluidity in organelle membranes, and perhaps also in myocardial plasma membranes. It is claimed that the observed effects of thyroid status on adrenergic sensitivities are not reflected in the number of $\beta$ - and $\alpha$-receptors. As measured by the binding of ${ }^{3} \mathrm{H}$-dihydroalprenolol at $37^{\circ} \mathrm{C}$ and ${ }^{3} \mathrm{H}$-dihydroergocryptine at $25^{\circ} \mathrm{C}$ to estimate the number of $\beta$ - and $\alpha$-receptors, respectively, crude preparations of heart plasma membranes from hypothyroid rats have only $20 \%$ as many $\alpha$-receptors and $70 \%$ as many $\beta$-receptors as normals. ${ }^{87}$ Thyrotoxicosis halves the number of $\alpha$-receptors and triples the $\beta$-receptors, while keeping the total number about constant. If hypothyroidism converts $\beta$ to $\alpha$, the total $\beta+\alpha$ should remain constant, but the unexplained measurements of receptors at different temperatures would seem to vitiate such a totalling, from the data of Kunos et al..$^{326-329}$ Besides, the shift from $\beta$ - to $\alpha$-receptor-induced rise in [cAMP] requires that the myocardium be contracting. ${ }^{326}$

Thermotropic properties of receptor-coupled adenylate cyclase activity in washed membrane particles of livers from normal rats may pertain to the above studies. Arrhenius plots of basal activity and activity stimulated in vitro by $\mathrm{F}^{-}$or $\mathrm{PGE}_{1}$ are linear, but plots of activity stimulated by epinephrine or glucagon are inflected. ${ }^{320}$ The plots of hormoneactivated rates are superimposable and unusual: both transition temperatures, $T_{t}$, are at $32^{\circ} \mathrm{C}$, whereas those seen with lipid-sensitive membrane-dependent enzymes are usually between $17^{\circ} \mathrm{C}$ and $22^{\circ} \mathrm{C}^{489} E_{\mathrm{a}}$ at $>T_{\mathrm{t}}$ is double the $E_{\mathrm{a}}$ at $<T_{\mathrm{t}}$, while the opposite is usually seen in membrane-dependent enzymes where decreased fluidity at the low temperatures increases the damping effects on activity. Because either saturating or lower [epinephrine] and [glucagon] produce identical Arrhenius profiles, Kreiner $e t a l .{ }^{320}$ think it unlikely that temperature changes alter receptor count, but rather that they act on a protein component common to the hormone-stimulated system but not to the $\mathrm{F}^{-}-$or the $\mathrm{PGE}_{1}$-stimulated 
systems. One wonders if these observations are explained by an increased number of $\beta$-receptors in hearts at the higher temperatures, and one hopes for direct measurements of thermotropic effects on adrenoceptors in these liver membranes.

Glucagon-receptors also respond to changes in thyroid status. Again there are apparently conflicting findings. In liver cell plasma membranes (frozen and thawed) from hypothyroid rats, Sperling et al ${ }^{569}$ report a $50 \%$ decrease in the number of glucagonreceptors; receptors have normal affinity for glucagon in vitro and bind less glucagon than normals at all [glucagon]; increased [glucagon] is necessary to produce a maximal increase in [cAMP], and the maximal [cAMP] produced is also half the maximum in euthyroid preparations. In intact hepatocytes from hypothyroid and euthyroid rats, Malbon et al. ${ }^{37}$ find that glucagon raises [cAMP] identically. When liver cell plasma membranes are prepared from rats treated with small doses of LT4, $0.05 \mu \mathrm{g} / \mathrm{g} /$ day $\times 14$ days, glucagonreceptors and [cAMP] responses are all normal. ${ }^{569}$ But in fat cells from rats treated about 10 times more vigorously with LT3, $\sim 0.1 \mu \mathrm{g} / \mathrm{g} /$ day $\times 8$ days, the number of glucagonreceptors and the response of [cAMP] to glucagon are more than twice-normal. ${ }^{374}$ It is difficult to say whether a lesser degree of hyperthyroidism or a greater responsiveness of fat cells contributes to the latter disparity, and whether plasma membrane integrity is involved in the former.

In vitro incubation of rat fat cells, ${ }^{322}$ thymocyte plasma membranes, ${ }^{538}$ or cat myocardium membrane preparations, ${ }^{352}$ with $1 \mathrm{nM}$ to $1 \mu \mathrm{M}$ LT3 stimulates cyclase activity.* Maximal increases occur at the $\mu \mathrm{M}$ concentrations, and as rapidly as 3-5 min. ${ }^{352,538}$ From congener efficacies and the effects of transcription blockers, T3 receptors (perhaps in plasma membranes) and protein synthesis mediate these increases in adenylate cyclase activity, except in cat heart. ${ }^{352}$ These in vitro actions eliminate possible mediation by receptors for other activators and indicate that thyroid hormones can activate independently. Thyroid hormones in vitro can also regulate the receptors for other hormones that are coupled to the adenylate cyclase system. Incubation of rat heart ventricle slices (but not isolated membranes) with LT3 quadruples the number of $\beta$-adrenergic receptors at $1.5-2 \mathrm{hr}$ with no immediate further increase; $15 \mathrm{pM}$ LT3 gives half-maximal stimulation, $0.1 \mathrm{nM}$ LT3 saturates $\beta$-agonist binding. ${ }^{303}$ Synthesis of protein is not involved. A $15-\mathrm{hr}$ incubation increases the number of $\beta$-receptors another 4 times, which does involve synthesis of new protein. Both the early and the later effects apear to be mediated by specific LT3 receptors. The rapid augmentation of cell surface $\beta$-adrenoceptors is attributed to an in vitro effect of $\mathrm{T} 3$ that somehow promotes their insertion from an existing cytosolic receptor pool. The increased number of $\beta$-receptors should increase [cAMP], if they are efficiently coupled to the adenylate cyclase, and phosphodiesterase activities are low.

\section{Cyclic Nucleotide Phosphodiesterases}

These diesterases are not in the plasma membrane but in the particulate membrane fractions that sediment at $>100,000 \times \mathrm{g}$ (microsomes), and in the supernatants. They are included here because there is evidence that thyroid hormone regulation of low- $K_{\mathrm{m}}$ cAMP phosphodiesterase activity complements and even predominates over thyroid regulation of adenylate cyclase activity in control of [cAMP]. Studies on thyroid effects on this heterogeneous group of enzymes are complicated by the existence of multiple enzyme 'forms', as shown by diesterase elution patterns during various chromatographic procedures, by their solubilities and kinetic properties, and by their specificities for substrate,

\footnotetext{
* Not so long ago, in vitro effects of thyroid hormones on oxidative phosphorylation were transferred from a 'physiologic' to a 'pharmacologic' category because they required micromolar concentrations. ${ }^{597}$ In Ref. 538 , the I nM [T3] needed for significant activation, and the $1 \mu \mathrm{M}$ needed for maximal activation, of adenylate cyclase are recognized to be well in excess of the $\sim 10 \mathrm{pM}$ [free T3] available in vivo; Segal et al. ${ }^{538}$ note that in 1985 the frequent need for 'supraphysiologic' concentrations of hormones to induce an in vitro effect that parallels their in vivo action is well known. They give as an example that most tissues of the rat require $0.1-100 \mu \mathrm{M}$ [epinephrine] to activate adenylate cyclase, whereas [epinephrine] in rat plasma is $\sim 1 \mathrm{nM}$.
} 
effector, and antibodies. ${ }^{28}$ Some of the forms may be convertible by solubilization from their loci in membranes, and by changes in phosphorylation status: insulin- and cAMPdependent phosphorylation doubles the $V$ of a phosphodiesterase in liver membranes.

Membrane-binding seems crucial for exhibiting phosphodiesterase thyroid-dependence, at least in fat cells. Hypothyroidism increases the activity and the $V$ of membranebound, but not of soluble or solubilized, low- $K_{\mathrm{m}}$ cAMP phosphodiesterase in rat adipocytes ${ }^{12,96,98,186}$ and in liver. ${ }^{200,407}$ Five days of T3-treatment of hypothyroids decreases fat cell phosphodiesterase $V$ by $-40 \%$. Adipocyte membranes contain only $10-20 \%$ of the total phosphodiesterase activity, and the 2- to 3-fold increase in activity, which is seen when hypothyroid preparations are disrupted in isotonic (but not hypotonic) buffers, occurs in a resolvable component uniquely sensitive to complete inhibition by excess cGMP; solubilization decreases the amount of this component. ${ }^{186}$ In 3T3-L1 adipocytes in culture, in contrast, both soluble and membrane-bound phosphodiesterase activity increases in cells in a low-T3 medium, and decreases in cells in a hyperthyroid ( $\sim 0.1 \mathrm{nM}$ T3) medium, as compared with cells in a medium containing serum from a euthyroid calf. ${ }^{129}$ Since epinephrine stimulates lipolysis in fat pads from hypothyroid rats hardly at all (c.f. $\sim 2.5$ times in euthyroids), and since theophylline-inhibition of phosphodiesterase restores the epinephrine stimulation in hypothyroid preparations, several workers propose that thyroid (and insulin) regulation of cAMP-dependent lipolysis in adipocytes is mediated by cGMP and the phosphodiesterase.

Destruction of membrane-dependence in hypotonic media seems to account for several reports that thyroid status does not alter low- $K_{\mathrm{m}}$ cAMP phosphodiesterase activity. In the liver, Morgan et al ${ }^{407}$ see no increase in $V$ of a phosphodiesterase that is solubilized from membranes of hypothyroid rats (although hyperthyroidism decreases $V$ ); and the phosphodiesterase activity of hypotonic homogenates of hepatocytes ${ }^{376 a}$ and of fat cells ${ }^{322}$ from T3-injected rats is at control levels. Thymocyte phosphodiesterase activity is nil $\pm \mathrm{T} 3$ in vitro. ${ }^{538}$ Fat pads minced in Krebs-Ringer-5\% albumin show similar phosphodiesterase activities in euthyroid and hyperthyroid rats. ${ }^{322}$ Cat heart preparations may be an exception; when homogenized in isotonic buffer, their phosphodiesterase activity remains unchanged after incubation with $5 \mu \mathrm{M}$ T3 although adenylate cyclase is activated, ${ }^{352}$ and Levy et al. ${ }^{352 a}$ find normal phosphodiesterase activity in sedimented membrane particles (medium isotonic) prepared from cats treated with T3 for up to 21 days. It should be noted that cats are unique among mammals in lacking a hepatic $\Delta 6$-desaturase to convert 18:2-CoA to $20: 4-\mathrm{CoA},{ }^{495 \mathrm{a}}$ and that there is no information on their cardiac membranes in different thyroid states. Thus, in hyperthyroid rat heart membranes prepared in isotonic buffer, cAMP phosphodiesterase activity is below normal levels. ${ }^{610} \mathrm{I}$ find no investigations of the membrane-dependence of the cGMP-sensitive form of phosphodiesterase in adipocytes, or the possible effects of the known changes in hepatic microsome fatty acyl unsaturation in hypothyroidism ${ }^{248}$ on phosphodiesterase activity.

\section{C. $\mathrm{Ca}^{2+}-$ ATPases}

Thyroid levels affect the amount of intracellular $\mathrm{Ca}^{2+}$ ions. Thyroid treatment markedly increases accumulation of $\mathrm{Ca}$ in liver and uterus, less so in skeletal muscle, ${ }^{642}$ and stimulates both efflux and influx of $\mathrm{Ca}^{2+}$ in liver in vitro without increasing binding or protein synthesis. ${ }^{624}$ Hypothyroidism depletes a slow-turnover pool of intracellular $\mathrm{Ca}^{2+}$ in isolated hepatocytes while leaving a fast-turnover pool unchanged; $48 \mathrm{hr}$ after T3-treatment the more inert pool remains unrestored although respiration increases. ${ }^{503}$ These observations reflect the actions of plasma membrane $\mathrm{Ca}^{2+}$-ATPase that partly dephosphorylates MgATP ${ }^{2-}\left(\mathrm{Ca}^{2+} / \mathrm{Mg}^{2+}\right.$-dependent ATPase $)$ to pump $\mathrm{Ca}$ from the cell. The concentration of free $\mathrm{Ca}^{2+}$ ions in cytoplasm ([free $\left.\mathrm{Ca}^{2+}\right]$ cyto) is the physiologically important effector. Calcium ion pumps located in several membranes normally maintain a low [free $\mathrm{Ca}^{2+}$ ]cyto against a $10^{4}$ times greater [free $\mathrm{Ca}^{2+}$ ] in serum-a higher gradient across the plasma membrane than that of any other ion. ${ }^{383}$ The $\mathrm{Ca}^{2+}$-ATPase of plasma membranes is supplemented by another $\mathrm{Ca}^{2+}$-ATPase in endoplasmic reticulum and by $\mathrm{Ca}^{2+}$-carriers in 
the mitochondrial inner membrane. The sarcoplasmic reticulum $\mathrm{Ca}^{2+}$-ATPase pumps cytoplasmic $\mathrm{Ca}^{2+}$ ions into the reticulum lumen where they are sequestered by binding to proteins, and the mitochondrial systems segregate $\mathrm{Ca}^{2+}$ in the matrix. Thus, [free $\mathrm{Ca}^{2+}$ ]cyto depends on three separate membrane systems. In resting non-muscle cells the plasma membrane $\mathrm{Ca}$-ATPase and the mitochondrial $\mathrm{Ca}^{2+}$-carriers predominate. Thyroid state modulates all these mechanisms; effects on the mitochondrial carriers are discussed in Section IV.B.8. In addition, $\mathrm{Ca}^{2+}$ efflux is stimulated by high extracellular $\left[\mathrm{Na}^{+}\right]$via a plasma membrane $\mathrm{Ca}^{2+}-\mathrm{Na}^{+}$exchange carrier; in this way, changes in rates of the thyroidsensitive plasma membrane $\mathrm{Na}^{+} \mathrm{K}^{+}$-ATPase (see Section III.D) and $\mathrm{Na}^{+}-\mathrm{H}^{+}$-antiporter (see Section III.E.2) can affect $\mathrm{Ca}^{2+}$ transports.

$\mathrm{Ca}^{2+}$ efflux across plasma membranes is best studied in red blood cell ghosts, in the absence of organelle membranes, protein synthesis, and nuclear actions. In hypothyroid rats, activity of erythrocyte membrane $\mathrm{Ca}^{2+}$-ATPase (at an unspecified temperature) is twice that in membranes from euthyroids. ${ }^{185}$ Because acceleration is confined to the calmodulin-dependent fraction of total activity and depends on iso-osmotic preparation of the membranes, thyroid deficiency is thought to increase the association of activated $\mathrm{Ca}$-calmodulin complex with the membrane rather than to induce $\mathrm{Ca}^{2+}$-ATPase (by some mechanism unusual to hypothyroidism). These findings, if applicable to other plasma membranes, are inconsistent with observed depletion of cell $\mathrm{Ca}$ in hypothyroidism. ${ }^{502}$ In contrast to an inverse relationship between activity and thyroid level, incubation of human erythrocyte membranes with $0.1 \mathrm{nM}$ T4 triples activity, and with $0.1 \mathrm{nM} \mathrm{T3}$, almost doubles activity measured at $37^{\circ} \mathrm{C}$. ${ }^{109}$ Because these preparations do not convert $\mathrm{T} 4$ to $\mathrm{T} 3$, this is a direct effect of $\mathrm{T} 4$, and one probably not mediated by known cell membrane T3-receptors. Rat erythrocyte $\mathrm{Ca}^{2+}$-ATPase does not respond to these low T3 and T4 concentrations, ${ }^{109}$ but incubation with $1 \mathrm{nM}$ T3 or $100 \mathrm{nM}$ T4 affects activity in a manner that depends on whether the rats have been fed a diet that contains corn oil or lard-and therefore that probably depends on membrane fatty acyl composition. ${ }^{164}$ The ratios 20:4 $46 / 18: 2 \omega 6$ and $18: 1 \omega 9 / 18: 2 \omega 6$ are $46 \%$ and $131 \%$ higher, respectively, in membranes from lard-fed rats than in membranes from corn oil-fed animals. T3, and less so $\mathrm{T4}$, inhibits $\mathrm{Ca}^{2+}$-ATPase activity in ghosts from the lard-fed group, but activates $\mathrm{Ca}^{2+}$-ATPase in ghosts from the corn oil group. Only $\mathrm{T} 3$ and T4 among thyroid analogues are active, so these effects might be receptor-mediated.

Activity of endoplasmic reticulum $\mathrm{Ca}^{2+}$-ATPase is directly proportional to thyroid state, at least when measured at $25^{\circ} \mathrm{C}$ in the specialized sarcoplasmic reticulum of muscle cells. Sarcoplasmic reticulum of heart ${ }^{354,585}$ and skeletal muscle ${ }^{151}$ of thyrotoxic rats and rabbits accumulates $\mathrm{Ca}^{2+}$ ions and has a $\mathrm{Ca}^{2+}$-ATPase activity twice as fast as preparations from euthyroid controls, but stores normal amounts of $\mathrm{Ca}^{2+}$. In rats, these $\mathrm{T} 4$ effects depend on protein synthesis, and are associated with increased passive permeability of the membranes to $\mathrm{Ca}^{2+}$ ions, increased sarcoplasmic reticulum phospholipid contents, and depleted 18:2 and 20:4 acyl contents that are replaced with 16:0 and 18:0 fatty acyls. ${ }^{354}$ In hypothyroid animals, $\mathrm{Ca}^{2+}$ uptake and $\mathrm{Ca}^{2+}$-ATPase activity measured at $25^{\circ} \mathrm{C}$ are $40-70 \%$ below normal levels. ${ }^{146,585}$ Treating hypothyroid rats (newborns from hypothyroid mothers) with $\mathrm{T} 3$ restores skeletal muscle membrane $\mathrm{Ca}^{2+}$ uptake slowly, by $50 \%$ in 5 days and $100 \%$ in 10 days, which suggests that synthesis of both protein and phospholipid are involved. ${ }^{156}$ Partly contrasting results are reported in thyrotoxic dogs, where cardiac sarcoplasmic reticulum accumulates and exchanges $\mathrm{Ca}^{2+}$ at $37^{\circ} \mathrm{C}$ faster than control preparations, while $\mathrm{Ca}^{2+}$-ATPase activity at that temperature is at normal levels and activity of the 'basic' $\mathbf{M g}^{2+}$-ATPase is depressed. ${ }^{421}$ The $\mathrm{Mg}^{2+}$-ATPase is not a sarcoplasmic reticulum enzyme but is contributed by contaminating plasma membranes and mitochondria. ${ }^{148}$ Without rigorous comparisons, it is not possible to decide whether the thyroid-insensitivity of the $\mathrm{Ca}^{2+}$-ATPase in a report by Naylor et al ${ }^{421}$ arises from differences in species or in temperature of measurement. However, temperature affects myocardial contractility differently in normal and abnormal thyroid conditions, and at least part of these contractile defects are attributed to altered in situ behavior of sarcoplasmic reticulum $\mathrm{Ca}^{2+}$-ATPase, through control of [free $\mathrm{Ca}^{2+}$ ]cyto. ${ }^{10,151,421}$ 
Isometrically contracting heart muscles from hyperthyroid cats, as compared with euthyroids, shorten faster, develop tension faster, and remain contracted for a shorter time. ${ }^{69}$ Muscles from hypothyroid cats show opposite changes. These abnormalities occur in isolated muscles kept at temperatures between $21^{\circ} \mathrm{C}$ and $32^{\circ} \mathrm{C}$, but the degree of difference at any one temperature varies with thyroid state-that is, thyroid levels regulate the thermotropic properties of contractility parameters. Since such behavior characterizes membrane-dependent processes, the molecular mechanisms are relevant here. Thyrotoxic hearts hypertrophy and hypothyroid hearts atrophy. Amounts of sarcotubular membrane per unit of myofibrillar volume remain constant while mitochondrial membranes increase in thyrotoxic rats. ${ }^{460}$ Actomyosin proliferates in thyrotoxic hearts and changes qualitatively, the proportion of the $V_{1}$ isomyosin rising to $90 \%$, as compared with $12 \% V_{1}$ and $88 \% \mathrm{~V}_{3}$ in euthyroids. ${ }^{138}$ In hypothyroids, only $\mathrm{V}_{3}$ heavy-chain myosin mRNA is expressed; $\mathrm{V}_{1}$ mRNA appears $4 \mathrm{hr}$ after such rats receive $\mathrm{T} 3$, and becomes $90 \%$ of total myosin mRNA at $72 \mathrm{hr}$. Thyrotoxic rabbit hearts at $21^{\circ} \mathrm{C}$ contract with decreased efficiency, measured as heat production per $\int$ (tension-time), which is equivalent to the ratio 'energy (ATP) utilized during cross-bridge cycling' per unit of work done. ${ }^{10}$ Most of the defect is ascribed to an increase in $V_{1}$, which cycles with a faster rate-limiting step and has higher myosin-ATPase activity than $V_{3}$. The remainder is attributed to the enhanced rates of depression of [free $\mathrm{Ca}^{2+}$ ]cyto and $\mathrm{Ca}^{2+}$ release by sarcoplasmic reticulum membranes during depolarization. In view of observations that absence of $V_{1}$ isomyosin accounts for $60 \%$ of the abnormal contractility in pressure-overload hypertrophy, ${ }^{10}$ presumably the major depletion of $V_{1}$ contributes comparably to the defects in hypothyroidism.

No studies of thermotropic properties of $V_{3}$ or $V_{1}$ isomyosins seem to be available. However, the sarcoplasmic reticulum $\mathrm{Ca}^{2+}$-ATPase depends on lipids. Delipidated $\mathrm{Ca}^{2+}$-ATPase is reactivated by either phospholipids or hydrocarbon anionic detergents, ${ }^{11}$ but requires phospholipids to transport $\mathrm{Ca}^{2+}{ }^{386}$ Cholesterol enrichment in liposomes also increases $\mathrm{Ca}^{2+}$-ATPase activity. ${ }^{373}$ The degree of fatty acyl unsaturation plays no important role in reactivation at room temperature, ${ }^{386}$ although it does alter $T_{\mathrm{t}}$ and $E_{\mathrm{a}}$ values in the inflected Arrhenius plots of activity. ${ }^{225,635}$ Experiments are still needed to decide if changes in sarcoplasmic reticulum lipids actually mediate part of the thyroid effects on $\mathrm{Ca}^{2+}$-ATPase activity.

\section{D. $\mathrm{Na}^{+} \mathrm{K}^{+}-$ATPase}

The $\mathrm{Na}^{+} \mathrm{K}^{+}$-ATPase protein complex is integral to cell membranes of vertebrates (except dog erythrocytes), where it maintains low $\left[\mathrm{Na}^{+}\right]$and high $\left[\mathrm{K}^{+}\right]$inside the cell $\left(\left[\mathrm{Na}^{+}\right]_{i}\right.$ and $\left.\left[\mathrm{K}^{+}\right]_{i}\right) . \mathrm{Na}^{+} \mathrm{K}^{+}-\mathrm{ATPase}$ is highly regulated. Highest activities are found in renal tubules and neural tissues, cells meant to exchange ions. Activity and regulation are, not unexpectedly, lipid-dependent, and in thyroid-sensitive tissues, thyroid-dependent. A major calorigenic role is proposed for this enzyme-transporter system, so its general relationship to lipids is relevant.

Several lines of evidence clarify $\mathrm{Na}^{+} \mathbf{K}^{+}$-ATPase membrane-dependency. Delipidation of plasma membranes with phospholipase $\mathrm{A}$ inactivates $\mathrm{Na}^{+} \mathrm{K}^{+}$-ATPase, but phospholipase $\mathrm{C}$ is relatively ineffective, ${ }^{78.595}$ which indicates that $\mathrm{PL}$ fatty acyls play a role in $\mathrm{Na}^{+} \mathrm{K}^{+}$-ATPase activity. Low concentrations of non-specific lipid-solubilizing detergents such as dodecyl sulfate and deoxycholate actually increase $\mathrm{Na}^{+} \mathbf{K}^{+}$-ATPase activity, whereas higher concentrations decrease activity. The activation may expose enzyme active sites otherwise covered by lipids, since the more lipid-soluble a cardiac glycoside is, the better it binds to rat brain $\mathrm{Na}^{+} \mathrm{K}^{+}$-ATPase. ${ }^{4}$ Heart sarcolemma $\mathrm{Na}^{+} \mathrm{K}^{+}$-ATPase seems to lack this lipid barrier, and binds the glycoside ouabain with extremely low affinity and dissociates from bound ouabain very rapidly; the enzyme is in lipids with a UI about twice that in other plasma membranes, which may contribute to its unusual behavior. Reconstitutions of delipidated $\mathrm{Na}^{+} \mathrm{K}^{+}-\mathrm{ATPase}$ in liposomes of acidic PL (PS or PG are those naturally present in plasma membranes) are most effective in restoring normal levels of activity, sensitivity to inhibition by ouabain, and biphasic Arrhenius plots of activity. ${ }^{79}$ 
Although PS seems so specific as to indicate it is essential for activity in brain or rabbit kidney enzyme, ${ }^{78,307,595} 1: 1 \mathrm{PC}+\mathrm{PE}$ and dioleoylphosphatidylglycerol are also effective with renal enzyme ${ }^{307}$ A 1981 review $^{535}$ concludes that activity requires no one PL polar head-group, and that the PL fatty acyls non-specifically provide a degree of lipid fluidity essential for $\mathrm{Na}^{+} \mathrm{K}^{+}$-ATPase activity, but the following evidence indicates that fatty acyls play a more specific role.

Fatty acyl composition of cell membrane phospholipids, when altered by diet, affects $\mathrm{Na}^{+} \mathrm{K}^{+}$-ATPase specific activity, the amount of $\mathrm{Na}^{+} \mathrm{K}^{+}$-ATPase, and $\mathrm{Na}^{+} \mathrm{K}^{+}$-ATPase membrane-dependency as shown by Arrhenius profiles and cooperativity of effectors. Chronic dietary deficiency of $\omega 6$-fatty acids partly replaces plasma membrane $\omega 6$-PUFA with $\omega 9$-PUFA, and increases $\mathrm{Na}^{+} \mathrm{K}^{+}$-ATPase activity and amounts-surprisingly, considering the paucity of $\omega 6$-PUFA in normal plasma membrane phospholipids and in the PS of plasma membranes. ${ }^{632}$ The number of $\mathrm{Na}^{+} \mathrm{K}^{+}$-ATPase molecules increases in liver and kidney particulate fractions. ${ }^{355,356}$ Activity increases in plasma membranes of hepatocytes, ${ }^{581}$ brain synaptosomes, ${ }^{586}$ heart sarcolemma, ${ }^{2}$ and in Ehrlich ascites tumor cells grown in mice so fed. ${ }^{565}$ Plasma membranes of cultured murine T-lymphocyte tumor EL4 cells incorporate saturated or unsaturated $(18: 1$ or $18: 2)$ fatty acids from their growth media to the extent of $20-50 \%$ of total PL fatty acyls. ${ }^{481}$ Neither saturated or unsaturated fatty acids affect ouabain-sensitive $\mathrm{Na}^{+} \mathrm{K}+$-ATPase specific activity at $37^{\circ} \mathrm{C}$ but the unsaturates decrease ouabain-binding or fluidity calculated from ESR of substituted 18:0-derivative probes. The 'molecular activity' ([ouabain-sensitive $\mathrm{Na}^{+} \mathrm{K}^{+}$-ATPase] [bound ouabain] $^{-1}$ ) is about 8 times higher in UFA-media, which is thought to represent the exposure of more existing $\mathrm{Na}^{+} \mathrm{K}^{+}$-ATPase ouabain-binding sites through direct molecular interactions between $\mathrm{Na}^{+} \mathrm{K}^{+}$-ATPase and unsaturated fatty acyl moieties. In Arrhenius plots of Ehrlich cell plasma membrane $\mathrm{Na}^{+} \mathrm{K}^{+}$-ATPase activity, the transition temperature is $\sim 14^{\circ} \mathrm{C}$ in EFA-deficiency (c.f. $22^{\circ} \mathrm{C}$ in controls); at temperatures $>14^{\circ} \mathrm{C}$, $\mathrm{Na}^{+} \mathrm{K}^{+}$-ATPase activity is greater than control activity while $E_{\mathrm{a}}$ is unchanged; at temperatures $<14^{\circ} \mathrm{C}, E_{\mathrm{a}}$ is twice the control value, ${ }^{565}$ indicating that the $\mathrm{Na}^{+} \mathrm{K}^{+}$-ATPase is in a less fluid lipid environment. The fatty acyl chain order parameters of plasma membranes from a hamster ovary cell line can be manipulated to change $\mathrm{Na}^{+} \mathrm{K}^{+}$-ATPase activity 10 -fold without altering the number of ATPase molecules, ${ }^{556}$ further indication that fluidity and activity are related. In heart sarcolemma from EFA-deficient rats, $\mathrm{Na}^{+} \mathrm{K}^{+}$-ATPase activity is greater than control levels at all temperatures below the transition temperature but the transition temperature is unchanged. ${ }^{2}$ Arrhenius profiles show that membrane lipids of rat brain interact progressively differently with $\mathrm{Na}^{+} \mathrm{K}^{+}$-ATPase during the postnatal phase of brain development, ${ }^{425}$ a period when brain cell respiration loses thyroid-dependence.

The PL fatty acyl composition of rat erythrocyte membranes resembles that of hepatocyte plasma membranes. The effects of EFA-deficiency on the $\mathrm{Na}^{+} \mathrm{K}^{+}$-ATPase of erythrocyte membranes have been examined through alterations in responses to $\mathrm{Na}^{+} \mathrm{K}^{+}$-ATPase effectors as measured by $n$, the Hill coefficient, as an index of degree of cooperativity (see Section IV.A). ${ }^{147}$ Since the specific activity of $\mathrm{Na}^{+} \mathrm{K}^{+}$-ATPase as measured in vitro depends strongly on the history and current state of the enzyme's exposure to effectors, ${ }^{4}$ these studies seem pertinent to considerations of thyroid regulation of $\mathrm{Na}^{+} \mathrm{K}^{+}$-ATPase. In red cells from rats fed the usual corn oil-supplemented diet, $n=2.9$ for $\mathrm{Na}^{+}$-activation of the ATPase; $\mathrm{Na}^{+}$-activation would need to be a high power term of $\left[\mathrm{Na}^{+}\right]$for amplification ${ }^{527-529}$ of the relatively small $\Delta\left[\mathrm{Na}^{+}\right]_{i}$ that regulates $\mathrm{Na}^{+} \mathrm{K}^{+}$-ATPase activity. Red cells from rats fed a fat-free diet show decreased $n$ values for $\mathrm{Na}^{+} \mathrm{K}^{+}$-ATPase activation by $\mathrm{Na}^{+}$or $\mathrm{K}^{+}$and for $\mathrm{Na}^{+} \mathrm{K}^{+}$-ATPase inhibition by $\mathrm{F}^{-}$. As the dietarily manipulated ratio of erythrocyte lipid unsaturated/saturated fatty acyls is increased, $n$ decreases. Cholesterol supplementation of the corn oil-containing diet increases $\boldsymbol{n}$ for $\mathrm{F}^{-}$-inhibition to 3.6. Thus, decreased membrane fluidity seems to increase the degree of cooperativity of this inhibitor, through the value of cell $\left[\mathrm{Na}^{+}\right]^{n}$.

Evidence that activity and amount of $\mathrm{Na}^{+} \mathrm{K}^{+}$-ATPase mediates thyroid-induced calorigenesis includes observed correlations between rates and ouabain-sensitivity of 
oxygen consumption, $\mathrm{Na}^{+} \mathrm{K}^{+}$-ATPase specific activity, $\mathrm{Na}^{+} \mathrm{K}^{+}$-ATPase amount, cell $\left[\mathrm{Na}^{+}\right]$and $\left[\mathrm{K}^{+}\right]$, tissue thyroid-sensitivity, and time-courses after administration of T3. ${ }^{125,219,269,270,566}$ For example, ouabain inhibits $30-40 \%$ of the respiration of liver or kidney slices from euthyroid rats, ${ }^{46,269,633}$ as well as the relatively depressed respiration in slices from hypothyroid rats; ${ }^{269}$ ouabain inhibits the stimulated respiration after T3-pretreatment ( 3 doses over 6 days) to about the levels in rats not treated with T3; and $\mathrm{Na}^{+} \mathrm{K}^{+}$-ATPase activity in homogenates is roughly proportional to slice respiration rates. ${ }^{269}$ Assumptions involved in concluding that $\mathrm{Na}^{+} \mathrm{K}^{+}$-ATPase mediates normal and pathological thyroid regulation of respiration include: (i) ouabain specifically binds and completely inhibits $\mathrm{Na}^{+} \mathrm{K}^{+}$-ATPase; (ii) ouabain-blockage of the $\mathrm{Na}^{+}$-pump affects no respiration-linked system other than $\mathrm{Na}^{+} \mathrm{K}^{+}$-ATPase; and (iii) mitochondrial respiratory control mechanisms respond 'normally' to $\Delta[\mathrm{ADP}]$ after the $\mathrm{Na}^{+}$-pump is inhibited by ouabain. These assumptions were critically reviewed in $1976^{225 a}$ and have been further challenged since.

(i) Ouabain-specificity: although ouabain did not directly inhibit respiration of isolated mitochondria from kidney, ${ }^{46}$ it inhibited respiration of brain mitochondria by up to $42 \% .{ }^{634}$ Less direct effects were seen in heart mitochondria prepared from guinea pigs pretreated with toxic doses of ouabain, where ADP did not fully accelerate respiration, ${ }^{346}$ perhaps because of interfering cation recompartmentation, as discussed by Gerrais et al ${ }^{168 a}$ These findings throw some doubt on the conclusion that ouabain-inhibition reveals a $40 \%$ $\mathrm{Na}^{+} \mathrm{K}^{+}$-ATPase contribution to thyroid respiratory stimulation. Moreover, tissue slices introduce artifacts into measurements of $\mathrm{Na}^{+} \mathrm{K}^{+}$-ATPase activity and amounts, ${ }^{42}$ and quantitative correlation between $\mathrm{Na}^{+} \mathrm{K}^{+}$-ATPase activity and amount is not valid when respiration and oubain-binding are measured in different ionic media. ${ }^{4}$ Some of the problems inherent in the use of slices are avoided in intact cell preparations, but there the $\mathrm{Na}^{+} \mathrm{K}^{+}$-ATPase contribution to thyroid calorigenesis is minimal or negligible: $<15 \%$ in striated muscles, ${ }^{42,91}$ and $5 \%$ in perfused liver ${ }^{27,154}$ and in isolated fat cells. ${ }^{143}$ Further, in broken-cell preparations of rat heart and skeletal muscle, ${ }^{20,548}$ rabbit renal tubules, ${ }^{543}$ and tissues of genetically obese $o b / o b$ mice, ${ }^{646}$ in vivo T3-pretreatment fails to induce $\mathrm{Na}^{+} \mathrm{K}^{+}$-ATPase. In the rabbits, T3-receptors are shown to be present and renal glycerol3-phosphate dehydrogenase and hepatic $\mathrm{Na}^{+} \mathrm{K}^{+}$-ATPase are concomitantly accelerated..$^{543}$ The $o b / o b$ mice lack a thyroid-responsive ouabain-sensitive $\mathrm{Na}^{+} \mathrm{K}^{+}$-ATPase although they have other thyroid-responsive systems, and York et al. ${ }^{646}$ discuss the possible contribution of the lack of $\mathrm{Na}^{+} \mathrm{K}^{+}$-ATPase-mediated energy consumption to accumulation of triglycerides that characterizes these animals. Hypothyroidism decreases ouabain-binding in the muscle membrane preparations; T3-treatment of either hypothyroid or euthyroid rats $(0.5 \mu \mathrm{g} / \mathrm{g}$ on days 1,3 , and 5 , killed on day 6) raises the ouabain-titer by markedly increasing the affinity of the $\mathrm{Na}^{+} \mathrm{K}^{+}$-ATPase for ouabain ${ }^{20,548}$-which is more consistent with T3-alteration of membrane lipids than with synthesis of $\mathrm{Na}^{+} \mathrm{K}^{+}$-ATPase.

(ii) Ouabain-blockage of the $\mathrm{Na}^{+}$-pump decreases cytosol [K+ ${ }^{+}$and [ADP], and increases $\left[\mathrm{Na}^{+}\right]$and $\left[\mathrm{P}_{\mathrm{i}}\right]$. It is the decrease in [ADP] that is postulated to account for the ouabain-inhibition of respiration. Additions of $\mathrm{K}^{+}$sufficient to prevent most of the decrease in $\left[\mathrm{K}^{+}\right]$in ouabain-bathed slices did not affect ouabain-insensitive respiration. ${ }^{269,270}$ However, through effects on proton entry into mitochondria, the mitochondrial inner membrane $\mathrm{Na}^{+} / \mathrm{H}^{+}$antiport would slow respiration when cytosol $\left[\mathrm{Na}^{+}\right]$increases, and the $\mathrm{P}_{\mathrm{i}} / \mathrm{OH}^{-}$antiport (which is equivalent to a $\mathrm{P}_{\mathrm{i}} / \mathrm{H}^{+}$symport) would stimulate respiration when $\left[P_{i}\right]$ increases.

(iii) Mitochondrial respiratory control: the premise that cytosol [ADP] always controls mitochondrial respiration in hypothyroid and hyperthyroid cells is overtaken by recent advances in understanding of control sensitivities in oxidative phosphorylation and the effects thereon of thyroid state changes (see Section IV.B.1). Briefly, [ADP] controls the increase in respiration during the State $4 \rightarrow$ State 3 transition only at intermediate rates of respiration, and not at all in State 3; the adenine nucleotide translocase contributes $29 \%$ of the control over State 3 respiration in mitochondria from normal rats, and only $19 \%$ 
in thyrotoxic rats. Why State 4 increases in thyrotoxicosis is discussed at length in Section IV.A. Furthermore, effects of altered $\mathrm{Na}^{+} \mathrm{K}^{+}-\mathrm{ATPase}$ activity on respiration must be measured in whole cells to preserve the existing cytosol [ADP]. But substrate efficacies in increasing cell respiration greatly modify mitochondrial State $4 \rightarrow$ State 3 transitions: glucose, which is conventionally used with whole-cell preparations, provides relatively little $(24 \%)$ oxidative support for the $\mathrm{Na}^{+} \mathrm{K}^{+}$-ATPase, while glucose plus fatty acids provides the most support (68\%). ${ }^{213}$ All this makes uncertain which control-contributing step (see Section IV.A) might link $\mathrm{Na}^{+} \mathrm{K}^{+}$-ATPase activity to respiration.

Ouabain-binding, immunoassay, and incorporation of $\left.{ }^{35} S\right]$ methionine or $\left[\gamma-{ }^{32} \mathrm{P}\right] \mathrm{ATP}$ into electrophoretically resolved $\alpha$ - and $\beta$-subunits ${ }^{103,357-359}$ have been used to show that T3-treatment of hypothyroid or euthyroid rats increases the number of $\mathrm{Na}^{+} \mathrm{K}^{+}$-ATPase molecules per $\mathrm{mg}$ protein in cells or membrane preparations. In vivo T3-treatment of hypothyroid rats also increases the amount of a mRNA for the $\alpha$-subunit of

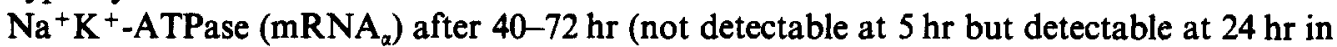
kidney and heart ${ }^{126,224,390,391}$ - this does not seem consistent with findings that $\mathrm{T} 3$ fails to increase the $V_{\max }$ of $\mathrm{Na}^{+} \mathrm{K}^{+}$-ATPase. ${ }^{20,548}$ Comparable lengths of time are required in a variety of cultured cells for $10-100 \mathrm{nM}$ T3 or $100 \mathrm{nM}$ T4 to increase $\mathrm{Na}^{+} \mathrm{K}^{+}$-ATPase activity, $\mathrm{mRNA}_{\alpha}$, or transmembrane resting potential, or to decrease cell $\left[\mathrm{Na}^{+}\right]{ }^{21,80,271,306}$ $\mathrm{Na}^{+} \mathrm{K}^{+}$-ATPase in different tissues may be genetically heterogeneous. ${ }^{144,145,391,532,590}$ Thus, although immunoassay and ouabain-binding show similar numbers of $\mathrm{Na}^{+} \mathrm{K}^{+}$-ATPase molecules in erythrocytes and myotubes, they show a 25 -fold discrepancy in fibroblasts and some other cells, and ouabain and antibody do not compete for binding. ${ }^{144,145}$ Only one or very few genes code for $\mathrm{Na}^{+} \mathrm{K}^{+}$-ATPase but mRNA levels vary in different tissues, suggesting control at transcriptional or post-transcriptional processes. ${ }^{533}$ Although translation-blockers prevent the induction (increased ouabain-binding) of the $\mathrm{Na}^{+} \mathrm{K}^{+}$-ATPase in HeLa cells grown in low $\left[\mathrm{K}^{+}\right]$medium, a transcription-blocker permits ouabain-binding to increase but decreases $\mathrm{K}^{+}$-influx activity ${ }^{47}$ The $\alpha$ - and $\beta$-subunits are initially inserted into endoplasmic reticulum membranes, ${ }^{126,224}$ the $\beta$ at the start of its synthesis, the $\alpha$ as late as $40 \%$-synthesized, depending on unspecified properties of the membrane. ${ }^{166}$ Normally, about half the newly synthesized $\mathrm{Na}^{+} \mathbf{K}^{+}$-ATPase later appears in the plasma membrane while the rest accumulates intracellularly. ${ }^{594}$ Thyroid hormones coordinately induce both $\alpha$ and $\beta$ subunits. ${ }^{358,359}$ Brain contains distinct $\alpha$ and $\alpha^{+}$forms; ${ }^{533,590}$ in developing brain, thyroid treatment hastens the appearance of both $\alpha$ (also found in kidney) and $\alpha^{+}$(the axolemmal form), while hypothyroidism blunts these increases. ${ }^{391,532}$

The slowness of these thyroid inductions, which begin at $24-48 \mathrm{hr}$ after T3 administration, is worth noting. The much earlier inductions of enzymes of lipogenesis and fatty acyl desaturation (see Section III.B.1) are not, from evidence available, directly connected with changes in the membrane-dependency of $\mathrm{Na}^{+} \mathbf{K}^{+}$-ATPase activity, although surrounding membrane lipids do affect activity (see above). Arrhenius plots of activity in myocardial microsomes from control or T3-treated rats each inflect at $24^{\circ} \mathrm{C}$ and have similar calculated values of $E_{\mathrm{a}}$, while $\mathrm{Na}^{+} \mathrm{K}^{+}$-ATPase activity is about $35 \%$ higher in the hyperthyroid preparations at temperatures from $13^{\circ} \mathrm{C}$ to $40^{\circ} \mathrm{C} .473$ Further, in crude homogenates of liver or kidney, the $E_{\mathrm{a}}$ of $\mathrm{Na}^{+} \mathrm{K}^{+}$-ATPase activity and of $\mathrm{Na}^{+} \mathrm{K}^{+}$-ATPase-dependent respiratory rates $\left(\mathrm{S}=\right.$ glucose) between $31^{\circ} \mathrm{C}$ and $40^{\circ} \mathrm{C}$ are unchanged in hyperthyroidism although $\mathrm{Na}^{+} \mathrm{K}^{+}$-ATPase and respiration are accelerated. ${ }^{488}$ Thus, one may conclude either that hyperthyroidism does not change the local lipid environment of the $\mathrm{Na}^{+} \mathrm{K}^{+}$-ATPase in the plasma membrane (Ref. 473; see Section III.A), or that any change that hyperthyroidism does effect in plasma membrane lipids does not alter $\mathrm{Na}^{+} \mathrm{K}^{+}$-ATPase thermotropic responses-perhaps in analogy with the failure of documented lipid changes in the mitochondrial inner membrane to alter those of the normally lipid dependent pyridine nucleotide transhydrogenase (see Section IV.B.9). What, then, might be the relationship between the T3-induced expression of genes, first and very early on those for a few lipogenic enzymes, and, later, those for the subunits of cell membrane $\mathrm{Na}^{+} \mathrm{K}^{+}$-ATPase? 


\section{E. Plasma Membrane Permeability}

Evidence now accumulates that $\mathrm{T} 3$-treatment first increases plasma membrane passive permeability to $\mathrm{Na}^{+}$and $\mathrm{K}^{+}$, and that increased cytosol $\left[\mathrm{Na}^{+}\right]$or decreased $\left[\mathrm{H}^{+}\right]$then mediates $\mathrm{Na}^{+} \mathrm{K}^{+}$-ATPase induction. High demands for $\mathrm{Na}^{+}$-transport generally induce $\mathrm{Na}^{+} \mathrm{K}^{+}$-ATPase, and are striking in renal tubules, especially those of the remaining kidney after uninephrectomy. ${ }^{299}$ The leakiness of cell membranes to $\mathrm{Na}^{+}$and $\mathrm{K}^{+}$correlates with the number of $\mathrm{Na}^{+} \mathrm{K}^{+}$-ATPase molecules in the membrane, and inhibition of $\mathrm{Na}^{+} \mathrm{K}^{+}$-ATPase leads to synthesis of more $\mathrm{Na}^{+} \mathrm{K}^{+}$-ATPase (see Ref. 225a). Dystrophic hamsters have sarcolemmae in heart and skeletal muscle that leak $\mathrm{K}^{+}$and have very high $\mathrm{Na}^{+} \mathrm{K}^{+}$-ATPase activity. In $\mathrm{HeLa}$ cells grown in low $\left[\mathrm{K}^{+}\right]$medium to induce the $\mathrm{Na}^{+} \mathrm{K}^{+}$-ATPase, $\left[\mathrm{Na}^{+}\right]_{i}$ increases to a maximum at $8 \mathrm{hr}$ and then starts to fall as ouabainbinding rises. ${ }^{47}$ An experimental doubling of passive $\mathrm{Na}^{+}$-influx into neuroblastoma cells more promptly increases $\mathrm{Na}^{+} \mathrm{K}^{+}$-pump activity that keeps $\left[\mathrm{Na}^{+}\right]_{i}$ constant. ${ }^{403}$

Although thyroid hormones increase the activity of $\mathrm{Na}^{+} \mathrm{K}^{+}$-ATPase measured in isolated plasma membrane preparations, in intact cells the passive permeability of the plasma membrane to $\mathrm{Na}^{+}$-influx and $\mathrm{K}^{+}$-efflux, rather than the $\mathrm{Na}^{+} \mathrm{K}^{+}$-ATPase capacity, limits any sustained increase in active pumping of $\mathrm{Na}^{+}$and $\mathrm{K}^{+} \cdot .^{13,14,154,202-204,403}$ Thyroid hormones $(0.2-1 \mu \mathrm{M}$ T3 or T4) (see footnote on page 232) applied in vitro increase, within the first hour, passive $\mathrm{Na}^{+}$-transport in the isolated bladder of the toad, ${ }^{187}$ and passive $\mathrm{K}^{+}$ permeability in frog skeletal muscle. ${ }^{195}$ Thyroid pretreatment in vivo stimulates permeability first. In diaphragm, and in heart and liver slices, passive permeability was initially rated a secondary target because thyroid treatment seemed to stimulate $\mathrm{Na}^{+} \mathrm{K}^{+}$-ATPase activity without changing cell $\left[\mathrm{Na}^{+}\right]$or $\left[\mathrm{K}^{+}\right] \cdot{ }^{270}$ However, T3-administration consisting of 3 doses of $0.5 \mu \mathrm{g} / \mathrm{g}$ given to normal rats over 6 days increases net passive flux of $\mathrm{K}^{+}$ and $\mathrm{Na}^{+}$in perfused isolated livers ${ }^{154}$ and in liver slices, ${ }^{203}$ and in isolated skeletal muscle. ${ }^{14,202}$ Very small doses $(0.01 \mu \mathrm{g} / \mathrm{g} /$ day $\times 14-16$ days $)$ given to hypothyroid rats increase $\mathrm{K}^{+}$-efflux but leave $\mathrm{Na}^{+} \mathrm{K}^{+}$-ATPase activity unchanged in liver slices ${ }^{204}$ and renal proximal tubules, ${ }^{73}$ whereas $0.5 \mu \mathrm{g} / \mathrm{g} /$ day for $3-7$ days first augments $\mathrm{K}^{+}$-efflux, then stimulates $\mathrm{Na}^{+} \mathrm{K}^{+}$-ATPase activity. Thus, increased passive mono-cation fluxes, rather than T3-occupancy of nuclear receptors, more immediately mediate thyroid activation of $\mathrm{Na}^{+} \mathrm{K}^{+}$-ATPase. ${ }^{202-204}$ Thyroid effects on passive $\mathrm{Na}^{+}$-influx in intact tissues tend to be obscured by the difficulty of differentiating $\left[\mathrm{Na}^{+}\right]_{\mathrm{i}}$ from extracellular $\left[\mathrm{Na}^{+}\right]$. The use of thyroid-sensitive cultured hepatocytes ${ }^{188}$ appears to circumvent this problem. Exposure to $10 \mathrm{nM} \mathrm{T3}$, renewed daily for 3 days, increases both passive $\mathrm{Na}^{+}$-influx and $\mathrm{K}^{+}$-efflux. ${ }^{272}$ The time course of such $\mathrm{T} 3$ actions is not yet available, but changes appear in days rather than in minutes.

$\mathrm{Na}^{+}$and $\mathrm{K}^{+}$can move passively across cell membranes by unmediated diffusion across the membrane lipids or by protein-mediated mechanisms. Non-mediated passive diffusion of $\mathrm{Na}^{+}$and $\mathrm{K}^{+}$across the phospholipids of the plasma membrane are discussed by Else and Hulbert ${ }^{131}$ as regulating calorigenesis in resting cells and being under the control of thyroid hormone levels. Membrane fatty acyl composition regulates cation leakage: $\mathrm{Na}^{+}$ and $\mathrm{K}^{+}$diffuse across artificial $\mathrm{PL}$ membranes prepared from lipids of normal rats $\left(E_{\mathrm{a}}=15-17 \mathrm{kcal} / \mathrm{mol}\right)$, and more readily across membranes from EFA-deficient animals $\left(E_{\mathrm{a}}=4 \mathrm{kcal} / \mathrm{mol}\right){ }^{405,465}$ Mediated transport has been studied more extensively.

\section{1. $\mathrm{Na}^{+}, \mathrm{K}^{+}$-Cotransporters}

The $\mathrm{Na}^{+}-\mathrm{K}^{+}-\mathrm{Cl}^{-}$cotransporter in the cultured hepatocytes is eliminated as a mediator in T3-stimulation of passive $\mathrm{K}^{+}$-influx because $\mathrm{T} 3$-treatment does not change its inhibition by furosemide. ${ }^{272}$ The glucose- $\mathrm{Na}^{+}$symporter in epithelial cells of small intestine and renal proximal tubules simulates a $\mathrm{Na}^{+}$-leak, and glucose is the conventional energy source for whole-cell preparations. The intestinal hexose- $\mathrm{Na}^{+}$symporter promptly activates the $\mathrm{Na}^{+} \mathrm{K}^{+}$-ATPase to maintain $\left[\mathrm{Na}^{+}\right]_{\mathrm{i}}$ homeostasis. ${ }^{261}$ However, neither hypo- nor hyperthyroidism changes $\mathrm{Na}^{+}$-dependent glucose uptake by renal tubule preparations. ${ }^{309}$ 
T3-incubation accelerates the insulin-dependent 2-deoxy-D-glucose transporter in cultured chick, embryo heart cells ${ }^{539}$ but the expected concomitant entry of $\mathrm{K}^{+}$would presumably decrease $\mathrm{Na}^{+} \mathrm{K}^{+}$-ATPase activity. Since blockage of synthesis of DNA, RNA, or proteins fails to affect $\mathrm{T} 3$-stimulation of deoxyglucose uptake in these cells, ${ }^{541}$ and the in vitro effectiveness of thyroid hormone analogues ranks almost similar to their efficacy in other in vivo assays, T3-receptors in the cell membrane are thought to mediate this glucose transport. Rat liver plasma membranes contain such specific, high-affinity sites ${ }^{479}$ but analogous sites do not appear to be connected with glucose- $\mathrm{Na}^{+}$entry into renal proximal tubules. T3-incubation with rat thymocytes first raises [cAMP] transiently, then increases 2-deoxyglucose uptake, and both effects require extracellular $\mathrm{Ca}^{2+} .540$ Epinephrine acts similarly but more rapidly, although no $\mathrm{Ca}^{2+}$ is required. Since alprenolol blocks the T3-effects as well as the epinephrine-effects, T3 seems to be acting here via $\beta$-adrenergic receptors.

\section{2. $\mathrm{Na}^{+}-\mathrm{H}^{+}$Antiporter}

The plasma membrane $\mathrm{Na}^{+}-\mathrm{H}^{+}$antiporter is thyroid-responsive. This antiporter has been recently recognized to be ubiquitous in plasma membranes of nucleated mammalian cells, where it exchanges $\mathrm{Na}^{+}$for $\mathrm{H}^{+}$one-for-one, electroneutrally, to contribute to cytoplasm pH $\left(\mathrm{pH}_{\mathrm{i}}\right)$ homeostasis (reviewed in Ref. 311). Cytoplasm $\left[\mathrm{H}^{+}\right]$is increased by mitochondrial and other oxidations. $\mathrm{Na}^{+}-\mathrm{H}^{+}$antiporter senses $\mathrm{pH}_{\mathrm{i}}$, perhaps through a site on its cytoplasmic face that binds $\mathrm{H}^{+}$and thereupon activates exchange. ${ }^{193}$ In effect, this transporter has a steep $v$ vs. $\mathrm{pH}_{\mathrm{i}}$ profile. Proton-efflux is driven in many cells by the pre-existing energy of the concentration gradient of $\left[\mathrm{Na}^{+}\right]$-outside $>\left[\mathrm{Na}^{+}\right]_{i}$, which has, in turn, been generated by the $\mathrm{Na}^{+} \mathrm{K}^{+}$-ATPase at the expense of ATP hydrolysis. The $\mathrm{Na}^{+}-\mathrm{H}^{+}$antiporter in brush border plasma membranes of epithelial cells of renal proximal tubules depends on thyroid status. ${ }^{518}$ The antiporter couples $\mathrm{Na}^{+}$-influx down the $\left[\mathrm{Na}^{+}\right]$-gradient (lumen $\rightarrow$ cell) with secretion of $\mathrm{H}^{+}$against the $\left[\mathrm{H}^{+}\right]$-gradient (cell $\rightarrow$ lumen). The basolateral plasma membrane of these renal cells contains a (thyroid-sensitive) $\mathrm{Na}^{+} \mathrm{K}^{+}$-ATPase that pumps $\mathrm{Na}^{+}$out of the cytoplasm into the blood stream while returning $\mathrm{K}^{+}$from blood to cytoplasm. Initial rates of $\mathrm{Na}^{+}-\mathrm{H}^{+}$antiporter activity in brush border membrane preparations from hypothyroid rats are half those in euthyroid controls; and $V$ is twice normal in membranes from hypothyroid rats made hyperthyroid by thyroid treatment for 3 weeks. At least $24 \mathrm{hr}$ is required for these effects of thyroid state change to appear. $\mathrm{Na}^{+}-\mathrm{H}^{+}$antiporter activity correlates highly with $\log [\mathrm{T} 4]$ in serum, under these conditions. Normally, increase in cytosol $\left[\mathrm{H}^{+}\right]$is a positive cooperative activator of $\mathrm{Na}^{+}-\mathrm{H}^{+}$antiporter, with a Hill coefficient, $n=1.43$; this is a relatively low $n$ to amplify the signal from observed $\Delta \mathrm{pH}_{\mathrm{i}}$ values of $0.1-0.6$, that correspond to a $\Delta\left[\mathrm{H}^{+}\right]$of $1.26-4$, during metabolic and developmental transitions in a variety of cells. Thyroid treatment affects neither $n$ nor $\left[\mathrm{H}^{+}\right]_{0.5}$. From these findings, Sacktor and Kinsella ${ }^{518}$ suggest that thyroid hormone either increases the number of $\mathrm{Na}^{+}-\mathrm{H}^{+}$antiporter per unit membrane or increases the exchange activity of existing $\mathrm{Na}^{+}-\mathrm{H}^{+}$antiporter. It is not yet clear what the relative rates and thyroid sensitivities are of the $\mathrm{Na}^{+}-\mathrm{H}^{+}$antiporter that puts $\mathrm{Na}^{+}$into the cytosol and the $\mathrm{Na}^{+} \mathrm{K}^{+}$-ATPase that pumps $\mathrm{Na}^{+}$out. Differential thyroid effects on cytosol $\left[\mathrm{Na}^{+}\right.$] may resemble the classical balancing of hepatic [cholesterol] through thyroid regulation of both synthesis and further metabolism.

Amiloride (a specific $\mathrm{Na}^{+}-\mathrm{H}^{+}$antiporter inhibitor) blocks $>80 \%$ of the entry of $\mathrm{Na}^{+}$ into either control or T3-incubated (for $72 \mathrm{hr}$ ) cultured liver cells, indicating that most of the $\mathrm{Na}^{+}$enters via the $\mathrm{Na}^{+}-\mathrm{H}^{+}$antiporter with or without added T3. ${ }^{272}$ However, T3 stimulates the remaining $<20 \%$ of $\mathrm{Na}^{+}$-entry (Table IV in Ref. 272 ), by $+86 \%$ with an active $\mathrm{Na}^{+}-\mathrm{H}^{+}$antiporter and by $+56 \%$ with inactive $\mathrm{Na}^{+}-\mathrm{H}^{+}$antiporter. It is not clear if $\mathrm{T} 3$ up-regulates this minor $\mathrm{Na}^{+}$-leakage path early enough and sufficiently to account for the later stimulation of $\mathrm{Na}^{+} \mathrm{K}^{+}$-ATPase specific activity or synthesis, or if this leakage is mediated or unmediated.

In considering how thyroid hormones may effect gene expression through alterations in 
membrane permeability to cations, it is of interest that hypothyroidism produces striking renal atrophy, and hyperthyroidism, hypertrophy. Thyroid state is only one of several regulators of the $\mathrm{Na}^{+}-\mathrm{H}^{+}$antiporter: among other activators are second messengers, growth-promoting factors, mitogens, phorbol diesters, insulin, glucocorticoids, $\mathrm{PGE}_{2}$. Several of these agents, as well as normal fertilization of sea urchin eggs, exert a common effect in raising $\mathrm{pH}_{\mathrm{i}}$, which is thought to mediate activation of DNA synthesis. ${ }^{72}$

Reports that T3-treatment leaves the membrane-dependency of the $\mathrm{Na}^{+} \mathrm{K}^{+}$-ATPase unchanged ${ }^{473}$ suggest that, if the hormone induces changes in plasma membrane lipids, they might affect the cation-leakage (via the $\mathrm{Na}^{+}-\mathrm{H}^{+}$antiporter and possibly across the $\mathrm{PL}$ as well) that initiates activation of the $\mathrm{Na}^{+} \mathrm{K}^{+}$-ATPase. The steps in a sequence of mechanisms, $\Delta[T 3, T 4] \rightarrow \Delta$ lipid composition $\rightarrow$ increased $C^{+}$permeability $\rightarrow \Delta\left[C^{+}\right]_{i} \rightarrow$ activation of gene expression $\rightarrow$ translation of the $C^{+}$-pump protein, would merit measurements of the control strengths, $C_{\mathrm{i}}$ (see Section IV.B). The step(s) $\Delta\left[C^{+}\right]_{i} \rightarrow$ gene expression is of great interest for the mechanisms of a number of instances where thyroid hormone first promotes activation of existing enzyme (e.g. by covalent modification; see Section II.B.1), then synthesis of that enzyme. ${ }^{235,236}$ Evidence discussed above indicates that $\mathrm{C}^{+}=\mathbf{H}^{+}$: the cell membrane system that exchanges $\mathrm{Na}^{+}$and $\mathrm{H}^{+}$is thyroid-responsive, and thyroid treatment decreases $\left[\mathrm{H}^{+}\right]_{\mathrm{i}}$, which in turn may stimulate transcription and synthesis of $\mathrm{Na}^{+} \mathrm{K}^{+}$-ATPase. Thyroid regulation of cell $\mathrm{H}^{+}$-circuits is discussed in the next section, on oxidative phosphorylation.

\section{MITOCHONDRIAL OXIDATIVE PHOSPHORYLATION}

Thyroid-state dependent changes in membrane lipid composition are likely to alter function in the inner membrane of mitochondria, which functions so completely through its properties as a barrier and as a matrix for deeply embedded transporter and enzyme proteins. Changes in inner membrane lipid composition brought about by a variety of means correlate with defects in oxidative phosphorylation..$^{92,240,370,393,489}$ Three examples can be given: (i) ethanol-feeding is of particular interest, in view of current observations that propylthiouracil, given in dosage used to convert human hyperthyroid subjects to euthyroid status, protects their livers against the lethal toxic effects of chronic excessive ethanol intake. ${ }^{455 a}$ Ethanol feeding produces the following changes in rats. It brings about a 'hyperthyroid' metabolic pattern in liver, ${ }^{273}$ but the mitochondrial dysfunction comprises decreases in both State 4 and State 3 respiration. It raises 18:2 and depresses 20:4 acyl proportions in liver mitochondrial $\Sigma$ PL, PC, and PE, ${ }^{157,395}$ but specifically lowers CL/PL ratios by $33 \%$ in a report by Miceli and Ferrell, ${ }^{395}$ and depletes $18: 2$ content in CL by $25 \%{ }^{626}$ It alters thermotropic properties of State 4 respiration, ${ }^{505,507}$ and decreases activities of the microsomal fatty acyl-CoA $\Delta 5$ - and $\Delta 6$-desaturases. ${ }^{426}$ However, for an opposing view, see Ref. 182. In contrast, hypothyroidism raises $C L / P L$ and the $C L$ contains normally high amounts of $18: 2$ acyls; ${ }^{248}$ one wonders if this is connected with the therapeutic effects of induced hypothyroidism. (ii) Adaptation to a cold environment is accompanied by changes in mitochondrial fatty acyl composition,,$^{370,468,478,489,575}$ and fails in hypothyroid rats. ${ }^{545}$ (iii) The most straight-forward demonstration of the degree to which mitochondrial $\omega 6$-PUFA distributions must be altered to affect coupling and the proton

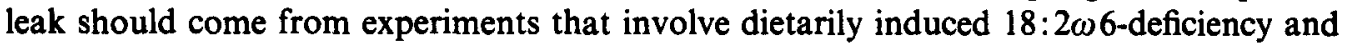
refeeding. Contradictory reports, and the importance of estimating the roles of these fatty acyls in thyroid mechanisms, make it advisable to examine effects of EFA-deficiency on oxidative phosphorylation here (see Section IV.E).

The degree of respiratory stimulation after thyroid treatment in vivo, and of respiratory depression in hypothyroidism, has been correlated with the amount of nuclear T3-receptors in different tissues. ${ }^{453,455}$ As shown in Table 5, spleen, testis, and brain from euthyroid rats have relatively few T3-receptors, as compared with liver, or on an absolute basis. Slices from those tissues (in which one cannot define respiration as being in State 4 or State 3) respire at about the rates of the thyroid-responsive tissues like liver, kidney, and heart, but T3-treatment does not stimulate their respiration, nor does hypothyroidism 
TABle 5. T3-Receptors, Respiration of Slices, and Mitochondrial Cardiolipins (CL) in Various Tissues

\begin{tabular}{|c|c|c|c|c|c|c|c|}
\hline \multirow[b]{2}{*}{ Tissue } & \multirow{2}{*}{$\begin{array}{l}\text { T3-receptors } \\
\text { (normalized }^{\mathrm{a}} \text { ) }\end{array}$} & \multicolumn{4}{|c|}{ Respiration $\left(\mu 1 \mathrm{O}_{2} / \mathrm{mg}\right.$ wet weight/hr) } & \multicolumn{2}{|c|}{ Mitochondrial $\mathrm{CL}^{\mathrm{d}}$} \\
\hline & & $\mathrm{Eu}^{\mathrm{b}}$ & $\Delta \% \mathrm{Eu}+\mathrm{T} 4$ & $\mathrm{H}^{\mathrm{c}}$ & $\Delta \% \mathrm{H}+\mathrm{T} 3$ & $\% \mathrm{CL} / \mathrm{PL}$ & $\% 18: 2 / \mathrm{CL}$ \\
\hline Liver & 1.0 & 1.29 & +62.4 & 1.01 & +63.8 & 14.8 & 59.4 \\
\hline Kidney & 1.47 & 3.62 & +37.8 & 3.11 & & 9.0 & 60.5 \\
\hline Heart & 0.45 & 0.76 & +24.2 & 0.47 & +200 & 20.0 & 74.8 \\
\hline Sk. muscle & & 0.57 & +28.6 & 0.45 & +47.7 & 12.5 & 23.3 \\
\hline Spleen & 0.18 & 1.45 & +10.8 & 1.43 & +1.6 & 7.3 & 48.6 \\
\hline Testis & 0.01 & 0.61 & -3.6 & 0.63 & -5.7 & 0.15 & 13.2 \\
\hline Brain & 0.24 & 1.01 & +4.3 & 0.93 & +2.4 & 1.6 & 10.3 \\
\hline
\end{tabular}

${ }^{a}$ ng T3 bound/mg DNA, normalized to liver. ${ }^{453}$ b(Ref. 183). '(Refs 24, 25, 312). ${ }^{d}(\operatorname{Refs} 253,632)$.

depress it. Both receptor count and respiratory response to altered thyroid levels correlate as well with the relative amounts of tissue cardiolipins (CL/PL ratio) and with the 18:2 acyl content of the CL. Thus, testis and brain PL include very little CL, and their CLs contain relatively little $18: 2$, as compared with the $10-20 \% \mathrm{CL} / \mathrm{PL}$ ratios in liver, kidney, and heart, and the 60-75\% 18:2 contents in those CLs (Table 5). Spleen is intermediate, with a $7.3 \% \mathrm{CL} / \mathrm{PL}$ ratio and $50 \% 18: 2$ in the CL. In contrast, heart PL combine the highest CL proportion with the highest percentage of 18:2 in the CL, and heart respiration responds to $\mathrm{T} 3$ treatment of hypothyroid rats most robustly despite a relatively low T3-receptor content. These data seem consistent with the 18:2 acyls of CL mediating effects of T3-receptor occupancy on tissue respiration. Respiratory responses to thyroid levels may depend on (mitochondrial) amount and 18:2 contents of $C L$, which may in turn depend on T3-receptor count, by way of T3-regulation of lipogenic enzymes.

Table 6 compares effects of thyroid levels on State 4 and State 3 respiration in rat heart mitochondria, with mitochondrial 18:2 content and CL/PL ratios. Hypothyroidism depresses respiration in either State, depending on the temperature of measurement. At $25^{\circ} \mathrm{C}$, hyperthyroidism accelerates either respiratory State, depending on the thyroid dose $\times$ time treatment schedule. ${ }^{474}$ In heart mitochondria, hypothyroidism leaves CL/PL ratios unchanged while $18: 2$ in $\mathrm{CL}$ decreases $22 \% ;{ }^{237}$ in liver mitochondria, hypothyroidism increases the ratio CL/PL by $72 \%$ but leaves the $18: 2$ content in CL unchanged. ${ }^{248}$ In liver mitochondria of thyrotoxic rats (normals injected with $\mathrm{T} 3,0.3 \mu \mathrm{g} / \mathrm{g} /$ day $\times 3$ days), CL/PL increases by $34 \%$ and CL $18: 2$ content decreases by $63 \% .{ }^{511}$ In our studies on thyrotoxics, T3 $(1 \mu \mathrm{g} / \mathrm{g} /$ day $\times 3$ days $)$ decreases liver mitochondrial 18:2 in PL by $26 \%$ (Table 1); in heart mitochondria, that dose does not alter CL/PL or 18:2 percentage in CL or PL. ${ }^{237}$ Thus, heart and liver mitochondrial CL compositions respond to thyroid levels differently; heart and liver mitochondrial functions differ, as is discussed in Section IV.D.

Membrane PL regulate a number of oxidative enzymes and related anion-carriers. ${ }^{335,523}$ Evidence from delipidation and reconstitution experiments also assigns a special role to cardiolipins in mediating thyroid effects on oxidative phosphorylation component proteins, as will be detailed. Cardiolipins are necessary for full reconstitution of several steps in oxidative phosphorylation but they are not sufficient without other PL. Cardiolipins bind some membrane proteins strongly and activate, or inhibit and make activity 'latent'. Distinctive properties of cardiolipins include the following. They contain 18:2 as about $55 \%$ (liver mitochondria) to $80 \%$ (heart mitochondria) of total fatty acyls, as compared to less than $15 \%$ in PCs and less than $10 \%$ in PEs. ${ }^{106,153,632}$ Cardiolipins are almost all in mitochondria, where they comprise $10 \%$ to $20 \%$ of the $\Sigma$ PL. The inner face of the inner membrane contains about $90 \%$ of the CLs of mitochondria, $65 \%$ of the PEs and $50 \%$ of the PCs. ${ }^{106}$ Hence, in heart mitochondria, the CL should be $40 \%$ of the inner face PL, and account for $85 \%$ of the inner face $18: 2$ acyls; in liver mitochondria, CL should be $20 \%$ of inner face PL and contain $60 \%$ of the 18:2. Cardiolipins have high activities in translocating cations across an organic phase separating two aqueous phases. ${ }^{612}$ Their extended hydrophilic group with its four long-chain hydrophobic moieties creates regions of small curvature in the membrane; ${ }^{394}$ perhaps that is why cardiolipins find their way to 


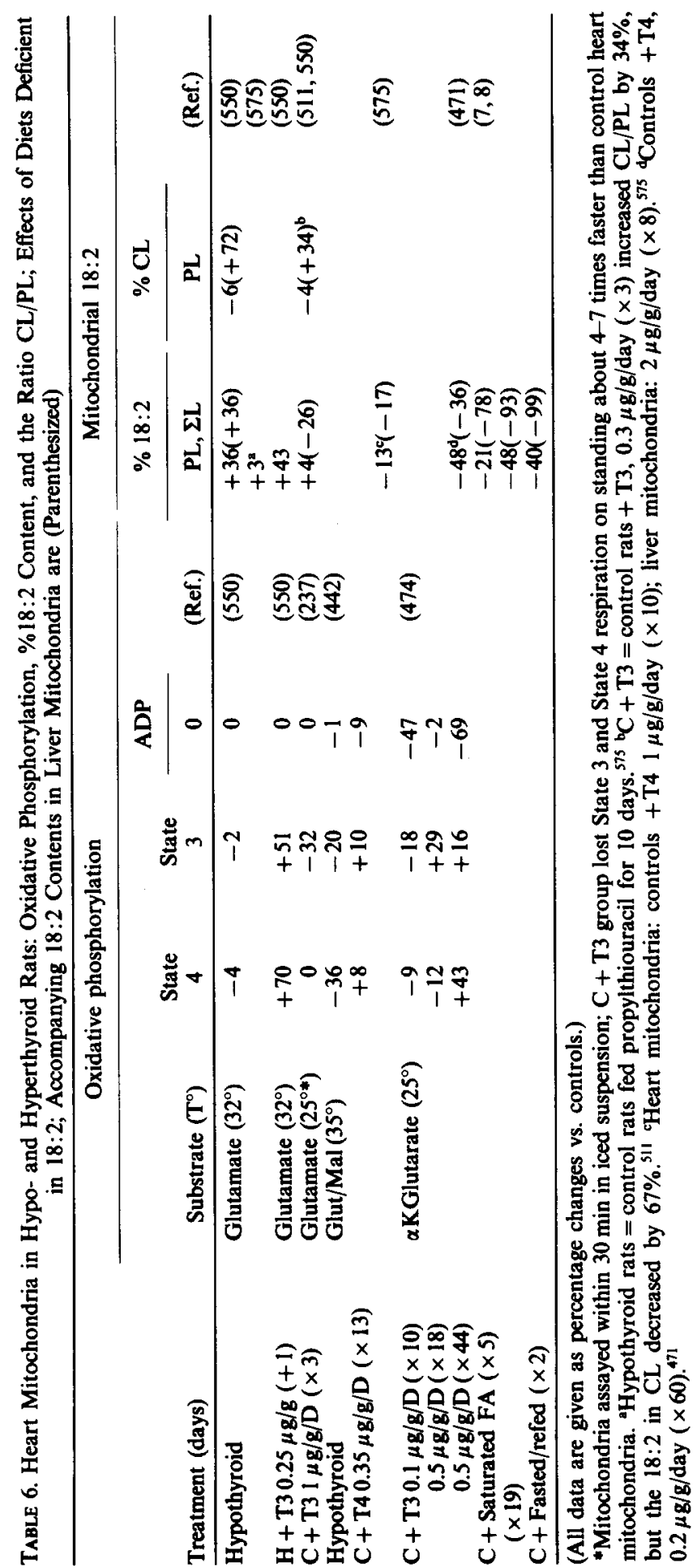


the inner surface of liposomes formed from mixtures of PL, ${ }^{410}$ and why CL digestion but not PE or PC digestion correlates with the disruption of inner membrane structure. ${ }^{18}$

Most workers agree that thyrotoxicosis accelerates, and hypothyroidism slows, mitochondrial State 4 respiration measured in vitro and its expression in vivo, the BMR. Three daily T4-injections given to normal rats accelerate liver mitochondrial State 4 respiration at $30^{\circ} \mathrm{C} 30-50 \%$, and decrease State 3 respiration slightly, with glutamate, $\alpha$-ketoglutarate, or $\beta$-hydroxybutyrate as substrate. ${ }^{245,246}$ Observations on State 3 respiration conflict: some find that thyroid state has no effect, ${ }^{245.246}$ and others that it alters State 3 in the same direction as State $4,61,62,551,552$ depending on the substrate; ${ }^{229}$ several report uncoupling of phosphorylation after high-dosage thyroid treatment in vivo, ${ }^{245,246,384,385}$ and others find normal $\mathrm{P} / \mathrm{O}$ ratios after lower doses. ${ }^{440,600}$ Recent non-invasive in situ measurements show decreased mitochondrial $\mathrm{P} / \mathrm{O}$ ratios in hearts of rats made hyperthyroid with small doses of T4. ${ }^{304}$

Despite the fact that thyroid treatment promotes gene expression and late synthesis of mitochondrial cytochromes, ${ }^{51,292,500,531,597,600}$ liver mitochondria from our normal and hypothyroid rats, and rats treated for short periods with hormone, show no major changes in either the relative amounts of proteins as measured by SDS-PAGE; ${ }^{81,83}$ in the amounts of cytochromes measured spectroscopically; ${ }^{51,61,62}$ or in protein/lipid ratios. ${ }^{248}$ Taken together with the multiplicity of thyroid-dependent membrane enzymes and transporters, and the small number of genes expressed after T3-treatment, these observations indicate to me the existence of one basic causal phenomenon, to others, multiple action loci. ${ }^{412,577}$ That phenomenon is temperature-sensitive.

Effects of altered lipid compositions on membrane proteins have been estimated through measurements of physical properties, and through effects of temperature and cooperativity of effectors on protein-mediated or lipid-mediated rate processes. Kinetic measurements have the advantage of measuring specific biological function. Since membrane lipids sense temperature changes, Arrhenius plots demonstrate how membrane enzymes and transporters respond to their lipid environment. ${ }^{393.489}$ Arrhenius profiles of State 4 respiratory rates in mitochondria from rat heart are normally linear from $10^{\circ} \mathrm{C}$ to $37^{\circ} \mathrm{C}$ whereas those of State 3 inflect at $\sim 25^{\circ} \mathrm{C}^{244}$ as do State 3 plots with liver mitochondria. ${ }^{302,347}$ Studies on State 4 respiration of liver mitochondria, especially in simple media, are difficult to find. However, it appears that State 3 respiration in this temperature range proceeds under at least two sets of mechanisms in either liver or heart mitochondria. Thyroid hormone deficiency in rats alters the inflected Arrhenius plot profiles obtained from liver or heart mitochondrial State 3 respiration, ${ }^{81-83,237,262,550}$ respiration-supported ATP-synthetase, ${ }^{81,82}$ adenine nucleotide translocations, ${ }^{234 a}$ and calcium ion transport (J. Peabody and F. L. Hoch, unpublished data). Hypothyroidism usually lowers activities and makes the Arrhenius profiles more linear at temperatures between $10^{\circ} \mathrm{C}$ and $37^{\circ} \mathrm{C}$. In some cases the hypothyroid and euthyroid plots intersect, making the temperature of measurement crucial for demonstrating hormone effect. Thus, observations that LT4-feeding does not change some mitochondrial systems measured at $37^{\circ} \mathrm{C}$ have been taken to indicate that concomitant thyroid responses at $<37^{\circ} \mathrm{C}$ are artifacts. ${ }^{118,119}$

Cooperativity of lipids and effectors on kinetics of membrane-dependent systems is also thyroid-dependent. Changes in the Hill coefficients of activations or inhibitions of allosteric membrane-bound enzymes denote a regulation of the enzyme through changes in membrane lipid unsaturation, effected by feeding rats EFA-deficient diets. ${ }^{147}$ The Hill number is said to detect changes in $E_{\mathrm{a}}$ of $\sim 0.75 \mathrm{kcal} / \mathrm{mol}$ as compared to $\sim 3 \mathrm{kcal} / \mathrm{mol}$ needed to shift the corresponding break in the Arrhenius profile. Wallach ${ }^{623}$ points out that, in a lattice mosaic model of biomembranes, protein-lipid subunits show positive cooperative interactions, and gel $\rightarrow$ liquid-crystal phase transitions can be effected either by raising temperature or by changing the ionic composition of the aqueous environment at constant temperature. The latter is the important phenomenon in membranes of homeothermic animal cells. The fact that a temperature change induces a lipid phase transition that alters function of a membrane enzyme or transporter, implies that ionic or effector changes would alter function similarly. An abnormal Arrhenius profile of a transporter 
caused by redistribution of membrane fatty acyls implies that the transporter will also respond abnormally in vivo to effectors. In poikilotherms, Arrhenius plots may directly reflect adaptations through altered membrane lipids.

If some thyroid effects are mediated through interactions of membrane lipids and proteins, altered interactions should be measurable in situ in biomembranes by spectroscopic methods. Here, apparently incompatible findings need conciliation for interpreting thyroid mechanisms. By ${ }^{2} \mathrm{H}-\mathrm{NMR}$, all the PL form a homogeneous phase: no lipid shell persists long enough to allow a stoichiometric complex. ${ }^{77,562}$ Intrinsic membrane proteins enveloped by the hydrophilic parts of amphiphilic lipids appear to respond only to the average properties of the PL, presumably at the level of amino acid side chain molecular rotation and translation, although Smith and Oldfield ${ }^{562}$ do not rule out specific interactions that are beyond current detection limits. Such methods do not appear to have been applied to possible thyroid influences on protein-lipid interactions as yet. On the other hand, membrane PL do affect membrane proteins, and all PL do not affect proteins similarly. In model membranes, PLs are not 'specifically' required for activity since detergents also activate, which is valuable information for understanding molecular mechanisms. However, membrane proteins do not see detergents in the mitochondrion, they see the natural amphiphiles PC, PE, CL, PS, PI, and perhaps some lyso-PLs. Evidence exists that proteins respond to changes in the proportions and fatty acyl compositions of these PLs - a biological selectivity if not a chemical specificity. And some thyroidresponsive proteins are difficult to delipidate, they retain stoichiometric amounts of CL, and CL effectively reconstitutes function after they are vigorously delipidated (see below).

\section{A. State 4 Respiration in Liver Mitochondria}

Recent insights into proton circuits, ${ }^{364,398-400,436,437}$ control mechanisms of oxidative phosphorylation, and their dependence upon membrane lipids explain some apparent discrepancies in studies on thyroid regulation. State 4 and State 3 respiration are differently controlled: State 4 depends on the barrier properties of membrane phospholipids. State 3 depends on several transporter proteins that participate in rate regulation, according to experiments (see Section B below) based on theoretical formulations of Refs 220, 221, 289, 290 and 527-529. The regulatory contributions of these transporters are tissue specific and strongly affected by the rate of respiration, the extra- or intramitochondrial location of the ADP-generating process, and the nature of their lipid matrix in the membrane. Thus, the respiratory control ratio, State $3 /$ State 4 , is hybrid, and State 4 respiratory rate is a more valid index of intactness of the inner membrane. ${ }^{436}$

Shears and Bronk ${ }^{551,552}$ show that LT4-treatment of normal rats, $8 \mu \mathrm{g} / \mathrm{g}$ body weight injected sc $24 \mathrm{hr}$ before killing, accelerates State 4 respiration and increases $\Delta p$ across the liver mitochondrial membrane, mainly by increasing $\Delta \psi(+13 \mathrm{mV})$ rather than $\Delta p H$ $(+3 \mathrm{mV})$ (Expt. 1, Table 7). The State 3 rate increases by $36 \%$; because the accompanying $\Delta p(113 \mathrm{mV}$; not shown) remains unchanged, the hormone was thought to accelerate State 3 and State 4 by different mechanisms. ${ }^{551,552}$ In hypothyroid rats killed $24 \mathrm{hr}$ after

TABLE 7. Rat Liver Mitochondrial State 4 and State 3 Respiration (ng atom O/min/mg); $\Delta \psi, \Delta p H, \Delta p(\mathrm{mV})$; Effects of LT4-injection, and the Presence of 'Decoupling' Agents

\begin{tabular}{|c|c|c|c|c|c|c|}
\hline & \multicolumn{4}{|c|}{ State 4} & \multirow{2}{*}{$\begin{array}{l}\text { State } 3 \\
\text { Resp. }\end{array}$} & \multirow[b]{2}{*}{ (Ref.) } \\
\hline & Resp. & $\Delta \psi$ & $\Delta p H$ & $\Delta p$ & & \\
\hline $\begin{array}{l}\left.\text { 1. Controls (succinate } 25^{\circ}\right) \\
+\mathrm{LT} 4(8 \mu \mathrm{g} / \mathrm{g}, 24 \mathrm{~h}) \\
\text { 2. Controls }\left(\text { glutamate } 30^{\circ}\right) \\
+ \text { Bilirubin }(24 \mu \mathrm{M}) \\
\left.\text { 3. Controls (succinate } 37^{\circ}\right) \\
+16: 0(10 \mathrm{nmol} / \mathrm{mg}) \\
\text { Controls }\left(\text { succinate } 25^{\circ}\right) \\
+18: 1(20 \mathrm{nmol} / \mathrm{mg})\end{array}$ & $\begin{array}{r}20 \\
30 \\
14 \\
41 \\
80 \\
175 \\
40 \\
180\end{array}$ & $\begin{array}{l}181 \\
194 \\
158 \\
164 \\
155 \\
160\end{array}$ & $\begin{array}{l}14 \\
17 \\
51 \\
65 \\
32 \\
20\end{array}$ & $\begin{array}{l}195 \\
211 \\
209 \\
229 \\
190 \\
180 \\
190 \\
190\end{array}$ & $\begin{array}{l}176 \\
175 \\
120 \\
180\end{array}$ & $\begin{array}{l}(551,552) \\
(584)\end{array}$ \\
\hline
\end{tabular}


T3-injection, $\Delta \psi$ increases $+7 \mathrm{mV} .^{618}$ However, no reports of which I am aware compare $\Delta p$ in liver mitochondria from euthyroid and hypothyroid rats, or from hypothyroids subjected to lesser dose $\times$ time treatments; and extrapolation from effects of thyroid on euthyroid rats is risky. Further, interpreting these data in terms of delocalized chemiosmosis poses a dilemma: if T4-injection acts as a protonophore, State 4 would accelerate but $\Delta p$ would fall; if T4 decreases the leak, $\Delta p$ will be maintained or increase but then State 4 should decrease.

Shears ${ }^{551}$ proposes that thyroid injection of normal rats might increase proton current non-ohmically under State 4 conditions by two effects: an increase in $\Delta p$ (presumably from electron-transport stimulation) enough to break down the proton resistivity of the thyroid-state-altered membrane lipids. The necessity for such postulates under State 3 conditions is disputed by Brand and Murphy. ${ }^{51}$ A non-ohmic, exponential relationship between $J_{\mathrm{H}^{+}}$and $\Delta p$ in rat liver mitochondria does become important at values of $\Delta p \simeq 200 \mathrm{mV}^{323,435,456}$ and pertains particularly to State 4 respiration, where $\Delta p$ is around $200 \mathrm{mV}$ : State 4 would thus be highly sensitive to changes in $\Delta p$. This exponential relationship is seen not only in intact mitochondria but also in protein-free liposomes made from mitochondrial lipids ${ }^{323}$ and in bilayer membranes consisting of pure phospholipids. $201,439,504$

The great disparity between induced changes in electron transport and $\Delta \tilde{\mu}_{\mathrm{H}^{+}}$is dismissed by Rottenberg and Hashimoto ${ }^{506}$ in their own experiments and those of Nicholls ${ }^{435}$ and Pietrobon and Caplan ${ }^{475}$ as artifactual because it occurs at $25^{\circ} \mathrm{C}$ but not at $37^{\circ} \mathrm{C}$. However, this thermosensitivity suggests that the fatty acyl chains of the membrane PL are involved. Saturated fatty acyl chains pack closely in membranes; unsaturated acyls kink and do not pack as closely, which permits water intercalation. Water molecules within the hydrophobic domain appear to conduct protons across pure PL membranes. ${ }^{52,201}$ Unsaturation at the $\omega 6$-position disturbs packing order mostly at the center of the bilayer. ${ }^{56,308}$ Thus $\omega 6$-unsaturated fatty acyls may play a special role in proton conductance. The observed redistribution of mitochondrial inner membrane $\omega 6$-PUFA contents (see Section II.A) may mediate at least some thyroid effects on proton flux in State 4 respiration.

Although proton current in State 4 is dissipated through the permeation of $\mathrm{H}^{+}$through membrane $\mathrm{PL}$, mediated cation $/ \mathrm{H}^{+}$antiport and anion $/ \mathrm{H}^{+}$symport also transport protons under usual conditions of assay. ${ }^{398}{ }^{400}$ Recommended and standard procedures ${ }^{76,135}$ measure State 4 in the presence of $P_{i}$ and $\mathbf{M g}^{2+}$ ions that are necessary only for subsequent assay of State 3 but also contribute to carrier-mediated proton transport in State 4 . State 4 respiration may well provide energy for such ion-pumping in vivo. However, adding mitochondria last to reaction mixtures designed for measurement of proton leaks through the PL of the membrane significantly depresses State 4 respiration because no unnecessary ions (e.g. $\mathrm{K}^{+}, \mathrm{Na}^{+}, \mathrm{Mg}^{2+}, \mathrm{P}_{i}$ ) are present and inhibitors are added to block ion transporters and the ATP-synthetase. ${ }^{44,67,323,438}$ Measurements under such conditions would help to decide whether changes in membrane phospholipid fatty acyl groups and/or proton-cotransport proteins mediate thyroid-state regulation of the proton leak and State 4 respiration. In particular, while a substrate carrier must support State 4 respiration, in effect, the carriers for tricarboxylates, glutamate, pyruvate, and dicarboxylates (see Ref. 51) introduce $\mathrm{H}^{+}$into the matrix; the (acyl)carnitine ${ }^{+}$carrier seems free from this objection. Besides, fatty acids are a more physiological substrate for mitochondrial oxidations; the succinate carrier is not required because succinate is generated and oxidized intramitochondrially. ${ }^{107}$

An alternative interpretation of the effects of thryoid state involves the existence of localized intramembrane proton circuits. ${ }^{637}$ In a mosaic chemiosmosis scheme, an intramembrane route coexists in parallel with the external proton circuit to regulate competition between energy-dependent processes. ${ }^{459,631}$ Decouplers are thought to introduce the intramembrane protons to transmembrane leakage routes. T4-injection (Expt. 1, Table 7) acts much like a decoupler does in vitro. Among the decouplers are free fatty acids ${ }^{506}$ and bilirubin ${ }^{584}$ (see Expts 2 and 3, Table 7). Chronic ethanol-feeding (which changes mitochondrial fatty acyl composition) also decouples. ${ }^{507}$ Decouplers accelerate State 4 
respiration but do not decrease $\Delta p$ in concentrations that uncouple; they produce loose coupling, ${ }^{342}$ as does thyroid treatment. ${ }^{226,246}$ They uncouple most in systems that require a high $\Delta p$ (reverse electron flow), less in those with an intermediate energy demand (ATP-synthetase), and minimally in those with low energy load (pyridine nucleotide transhydrogenase). ${ }^{505,506}$ Decouplers act in at least stoichiometric quantities; the amount of free fatty acids required for maximal uncoupling far exceeds the content of proton pumps ( $1 \mathrm{nmol} / \mathrm{mg}$ protein), and the amount of bilirubin needed is even greater. Protonophores act in catalytic quantity. ${ }^{438}$ Normal rat liver mitochondria contain little T4 or T3, about $0.4 \mathrm{pmol} / \mathrm{mg}$ protein; in hypothyroidism, hormone content decreases by $80 \%$; after hormone injection, in hypothyroids, the mitochondrial hormone increases 100 -fold in $2 \mathrm{~min}$, and in euthyroids 550 -fold by $3 \mathrm{hr} .{ }^{116,228}$ These data speak strongly against the normal amount of hormone molecules acting directly as decouplers, although hormone accumulation after thyroid injection in euthyroid rats does approach stoichiometric values. On the other hand, thyroid treatment redistributes the phospholipid fatty acyl composition in bulk, and the fatty acyl chains seem eligible as decouplers.

Proton leakage under State 4 conditions has been quantitated in terms of the amount of added FCCP that is equivalent to the leak at $[\mathrm{FCCP}]=0 .{ }^{194}$ In normal liver mitochondria in a reaction mixture containing succinate/malate and the traditional irrelevant ionic ingredients $\left(\mathrm{K}^{+}, \mathrm{Mg}^{2+}, \mathrm{P}_{\mathrm{i}}\right.$, and even $\left.1 \mathrm{mM} \mathrm{ATP}\right)$ at $25^{\circ} \mathrm{C}$, the extrapolated value is about 12 pmol FCCP per mg protein (State 4, Table 8). Liver mitochondria from hypothyroid rats leak protons under these State 4 conditions abnormally slowly; one injection of LT3, $0.4 \mu \mathrm{g} / \mathrm{g}$ body weight, partly restores the leak in $24 \mathrm{hr}$ (more completely when measured by State 4 respiration). Verhoeven et al ${ }^{618}$ recognize that their uncoupler titrations do not reveal the process responsible for proton leakage that is affected by the hormone, other than that the presence of oligomycin eliminates participation by the ATPase. The rate of State 4 respiration itself is more informative on mechanism.

From separate experiments, State 4 respiration is, in general, inversely proportional to

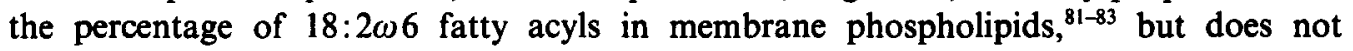
correlate with contents of $20: 4 \omega 6$ or $22: 6 \omega 3$ or the overall UI (membrane fluidity). To determine if a rigorous correlation exists, concomitant measurements of State 4 respiration under appropriate conditions, and fatty acyl compositions, are still needed, especially as functions of dose $\times$ time after thyroid treatment of euthyroid and hypothyroid animals. Such a correlation casts $18: 2$ as a 'proton-plug' that decreases transmembrane proton leakage; and/or as a 'coupler' that conducts intramembrane protons from the redox pump directly to the ATP-synthetase but away from the leak that connects intramembrane protons with the bulk phases; or as a positive effector for the redox pump that increases $\Delta p$, particularly at the cytochrome $b c_{1}$ segment (Table 8). These considerations imply that thyroid state regulates both the incorporation of 18:2 into liver PL (Table 2) and the desaturations of $18: 2$ to $20: 4$ (see Section II.I) to adjust the inner membrane content of 18:2 fatty acyls, and thereby maintains efficient proton transport for oxidative phosphorylation. Desaturation of linoleoyl acyl groups would then supply 20:4 for prostanoid

TABLE 8. Liver Mitochondria: The Effects of Thyroid State on the Proton Leak During State 4 Respiration, on the Distribution of Control Strengths $\left(C_{\mathrm{i}}\right)$ during State 3 Respiration, and on Respiration Rates (Succinate/Malate, 25-26 ${ }^{\circ} \mathrm{C}$ ); From Refs 194,618

\begin{tabular}{|c|c|c|c|c|}
\hline & Step & Normal & Нypo & $\mathbf{H}+\mathbf{T}$ \\
\hline State 4 & $\begin{array}{l}\text { Proton leak (pmol FCCP/mg) } \\
\left.\text { (ng ion } \mathrm{H}^{+} / \mathrm{min} / \mathrm{mg}\right)^{*}\end{array}$ & $\begin{array}{l}11.8 \\
318\end{array}$ & $\begin{array}{c}4.5 \\
198\end{array}$ & $\begin{array}{c}5.8 \\
276\end{array}$ \\
\hline State 3 & $\begin{array}{l}\text { Respiration (ng atom } 0 / \mathrm{min} / \mathrm{mg} \text { ) } \\
\text { ADP/ATP carrier }\left(C_{\mathrm{i}}\right) \\
\text { Dicarboxylate carrier }\left(C_{\mathrm{i}}\right) \\
\text { Cytochrome } b c_{\mathrm{i}}\left(C_{\mathrm{i}}\right) \\
\text { Cytochrome oxidase }\left(C_{\mathrm{i}}\right) \\
\text { Proton leak }\left(C_{\mathrm{i}}\right) \\
\Sigma\left(C_{\mathrm{i}}\right)\end{array}$ & $\begin{array}{r}305 \\
0.29 \\
0.33 \\
0.03 \\
0.17 \\
0.04 \\
0.86\end{array}$ & $\begin{array}{c}227 \\
0.18 \\
0.21 \\
0.21 \\
0.14\end{array}$ & $\begin{array}{r}338 \\
0.27 \\
0.34 \\
0.14 \\
0.18 \\
\\
0.93\end{array}$ \\
\hline
\end{tabular}

* Calculated from State 4 respiration (not shown), and $\mathrm{H}^{+} / \mathrm{O}=6$. 
synthesis rather than for a role in oxidative phosphorylation, and would decrease 18:2 acyl content and thereby accelerate proton leakage.

One injection of LT4 into hypothyroid rats progressively increases State 4 respiration at $25^{\circ} \mathrm{C}$ in liver mitochondria from animals killed $2-180 \mathrm{~min}$ later, 228,229 a period when PUFA contents decrease and saturated fatty acyls increase (Table 1). We in effect estimated proton leakage by titrating liver mitochondria with dinitrophenol (glutamate; in the presence of $\mathrm{K}^{+}$and $\mathrm{P}_{\mathrm{i}} ; 25^{\circ} \mathrm{C}$ ) and measuring the 'sensitivity' of the increase in respiration by the calculated Hill coefficient, $n{ }^{147,527-529}$ In mitochondria from hypothyroid rats, $n$ is depressed below normal values; injection of LT4, $5 \mu \mathrm{g} / \mathrm{g}$ body weight, increases $n$, within minutes in hypothyroid rats and in $6 \mathrm{hr}$ in normal rats (Table 9). Thus, the DNP-sensitivity of the proton leak relates directly to thyroid state and to State 4 respiration. DNP is partly ionized at $\mathrm{pH} 7.4$ and so titrates respiration non-linearly because the first molecules added remain in the aqueous phase; at $\mathrm{pH}$ 6.5, DNP titrates respiration linearly, like FCCP at pH $7.4^{194}$ (which dissolves completely in the membrane lipids even at low [FCCP]). ${ }^{222,438}$ Hill numbers thus reflect the relative partition of DNP into the membrane lipids, which is apparently less in hypothyroid mitochondria than in normals, and increases rapidly after LT4-treatment. The altered fatty acyl composition of mitochondria in hypothyroid animals would thus seem to be involved in the observed changes in DNP partition and effectiveness. DNP injected into euthyroid rats increases mitochondrial calorigenesis in $30 \mathrm{~min}$, as measured by the BMR. DNP-induced calorigenesis decreases markedly in severely hypothyroid rats, and a sub-calorigenic dose of LT4 restores it within $6 \mathrm{hr}^{227}$ These in vivo effects may reflect the effectiveness of DNP on mitochondrial proton leakage.

I do not known why the addition of albumin reverses the increased proton leak that occurs 30 min after T4-injection. ${ }^{228,247}$ Albumin has been used to 'improve' mitochondrial respiratory control in normal mitochondria ${ }^{600}$ by decreasing the proton leak. If altered fatty acyl composition mediates the T4-induced leak increase, albumin should have no effect-albeit no one has reported what albumin does to mitochondrial fatty acyls. It seems more likely that the affinity of albumin for cations, fatty acids, and thyroid hormones is involved. Although albumin partly removes hormone from mitochondria of euthyroid and hormone-treated hypothyroid rats, there are reasons against thinking that T3 or T4 acts directly as a protonophore, i.e. in catalytic amounts. Measurements in the absence of unneeded permeant ions might provide answers.

\section{B. State 3 Respiration in Liver Mitochondria}

Calculations of control stength, $C_{\mathrm{i}}$, from titrations of respiration with inhibitors specific for component steps, show that State 3 respiration (succinate/malate; $25^{\circ} \mathrm{C}$ ) in liver mitochondria from normal rats is regulated almost completely $\left(\Sigma C_{\mathrm{i}}=0.82\right)$ by three protein-mediated transports: the dicarboxylate and the ADP/ATP carriers together account for a $C_{\mathrm{i}}=0.62$, and the electron carriers at the cytochrome $a a_{3}$ segment for a $C_{\mathrm{i}}=0.17$ (Table 8). The $b c_{1}$ site and the proton leak regulate minimally. ${ }^{49,167,194}$ At less than

TABLE 9. Hill Coefficients ( $n$ ) of the Dinitrophenol-Titrations of Respiration, and State 4 Respiration (ng atom O/min/mg Protein; $\mathrm{S}=$ Glutamate, $25^{\circ} \mathrm{C}$ ), in the Liver Mitochondria of Normal and Hypothyroid Rats; the Effects of One ip Injection of LT4, $5 \mu \mathrm{g} / \mathrm{g}$; Recalculated from Refs 229, 230

\begin{tabular}{ccccccc}
\hline \multicolumn{3}{c}{ Normal rats } & & \multicolumn{3}{c}{ Hypothyroid rats } \\
\cline { 1 - 2 } \cline { 5 - 7 } $\begin{array}{c}\text { Time after T4 } \\
\text { (hr) }\end{array}$ & $n$ & $\begin{array}{c}\text { State 4 } \\
\text { Resp. }\end{array}$ & & $\begin{array}{c}\text { Time after T4 } \\
\text { (min) }\end{array}$ & $n$ & $\begin{array}{c}\text { State 4 } \\
\text { Resp. }\end{array}$ \\
\hline 0 & 1.22 & 9.5 & & 0 & 0.79 & 6.7 \\
3 & 1.22 & 9.2 & & 2 & $0.98^{*}$ & $8.1^{*}$ \\
6 & $1.50^{*}$ & 9.8 & & 30 & $1.06^{*}$ & $7.9^{*}$ \\
24 & $1.59^{*}$ & $12.2^{*}$ & & 180 & $1.06^{*}$ & $7.9^{*}$ \\
48 & $2.10^{*}$ & 10.1 & & & & \\
\hline
\end{tabular}


maximal respiratory stimulation by external ADP, the ATP/ADP carrier $C_{\mathrm{i}}$ increases up to about 0.5 and that for the dicarboxylate carrier decreases to $0.2,{ }^{49,167}$ but the effects of thyroid state have been reported only for maximal State 3 respiration. In State 3 the $P_{i}$ carrier $C_{\mathrm{i}}$ and the ATP synthetase ${ }^{49} C_{\mathrm{i}}=0$. A much different $C_{\mathrm{i}}$ distribution regulates State 3 in other than adult rat liver mitochondria (see Sections IV.C and D).

Hypothyroidism decreases the rate of State 3 respiration while markedly increasing the $C_{\mathrm{i}}$ of the cytochrome $b c_{1}$ segment at the expense of the control strengths of the adenine nucleotide translocase and the succinate carrier: electron transport through $b c_{1}$ is slowed relative to the capacities of the two carriers that normally regulate most (Table 8 ). The $\Sigma C_{\mathrm{i}}=0.74$; among the steps not measured are the succinate dehydrogenase, the $\mathrm{P}_{\mathrm{i}}$ transporter, the various cation transporters, and the proton leak. The proton leak does not seem a good candidate for gaining in control over State 3, since it is markedly depressed in hypothyroidism.

LT3-injection restores the State 3 rate and the $C_{\mathrm{i}}$ values toward control levels, although the $b c_{1}$ segment still contributes more than normally. Conspicuously unresponsive to thyroid state is the minor $C_{\mathrm{i}}$ of the cytochrome oxidase step. The dose of T3 used does not uncouple, and does not increase proton leakage to become a significant regulator of State 3 respiration. We still do not know what doses of thyroid hormone large enough to uncouple do to mitochondrial lipid composition, but dietary deficiency of 18:2 fatty acid, especially when accompanied by feeding of saturated or $\omega 3$-unsaturated fatty acids, severely depletes mitochondrial 18:2 fatty acyls, and uncouples, albeit inconsistently (see Section IV.E).

The energy-dependent fluorescence yield of 1-anilino-8-naphthalene under State 3 conditions (succinate, $30^{\circ} \mathrm{C}$ ) decreases in liver mitochondria from hypophysectomized rats and is restored after LT4-treatment. ${ }^{372}$ Since this probe presumably reports on the polar-nonpolar interface region of the inner membrane, these data indicate that thyroid affects State 3 through membrane phospholipids. Thyroid-responsive liver mitochondrial proteins that regulate State 3 respiration involve cardiolipins: the transporters of (1) ADP/ATP, (2) substrates, and electrons at (3) the cytochrome $b c_{1}$ segment. (4) The cytochrome $a a_{3}$ step also regulates and is CL-dependent, but not thyroid-responsive. Other proteins that may not regulate respiration in liver mitochondria (although some regulate in mitochondria of neonatal liver and heart; see Section IV.C and D), but also involve CL include (5) the $P_{i}$ carrier, (6) substrate dehydrogenases, (7) the ATP synthetase, (8) the $\mathrm{Ca}^{2+}$ transporter, and (9) the pyridine nucleotide transhydrogenase.

\section{ADP/ATP Carrier}

The observed slowing in adenine nucleotide translocation in mitochondria from livers of hypothyroid rats ${ }^{483}$ is claimed to depress ATP synthesis ${ }^{578}$ because the translocator is 'rate-limiting'. ${ }^{108}$ Indeed, the ADP/ATP carrier contributes to respiratory regulation in euthyroid rat hepatocytes suspended in ionic buffer, $\mathrm{Ca}^{2+}$ ions, bovine serum albumin, and lactate + pyruvate, at $37^{\circ} \mathrm{C} .{ }^{122 a}$ Eighty percent of the respiration is mitochondrial, and $70 \%$ of that represents State 3 respiration, for which the $C_{i}=0.26$ (c.f. 0.29 in isolated mitochondria, Table 8). Hyperthyroidism is thought to accelerate the ADP/ATP carrier in vivo, from studies on perfused rat livers where cytoplasm ATP/ADP ratios are twice normal even though ATP is more rapidly used for enhanced urea and glucose synthesis, and $\mathrm{Na}^{+}$-pumping. ${ }^{542}$ These claims are contradicted by the observed decrease of the $C_{\mathrm{i}}$ in hypothyroids and the restoration of the $C_{\mathrm{i}}$ after T3-treatment (Table 8; see also Ref. 51). Claims that the ADP/ATP carrier of adult beef heart mitochondria is the LT3-receptor that regulates respiration ${ }^{578}$ are countered by additional facts: heart mitochondrial respiration is not regulated under any experimental conditions by this carrier, ${ }^{117}$ and no information is available that thyroid state affects the heart translocase; the moles of thyroid hormone bound ${ }^{578}$ and found ${ }^{166}$ in liver mitochondria are at least 3 orders of magnitude less than the $\sim 1 \mathrm{nmol}$ of carrier per mg protein; $;^{45}$ the putative similarity between amino acid compositions (not sequences), and perhaps LT3-binding, of the carrier and the 
receptor may be no more than is to be expected from two lipophilic membrane proteins; and by other considerations. ${ }^{51,340,619}$

The abnormal thermotropic properties of the ADP/ATP carrier in liver mitochondria from hypothyroid rats ${ }^{234 a}$ suggest that altered fatty acyl composition might also change carrier regulation by other effectors. However, in normal mitochondria the $C_{\mathrm{i}}=0.29$ at $25^{\circ} \mathrm{C}$ (Table 8) and 0.31 at $37^{\circ} \mathrm{C} ;{ }^{.251}$ in hypothyroid mitochondria, $C_{\mathrm{i}}=0.18$ at $25^{\circ} \mathrm{C}, 0.17$ at $37^{\circ} \mathrm{C}$. Brief LT3-pretreatment of hypothyroid rats leaves the $C_{i}$ at $37^{\circ} \mathrm{C}$ unchanged but restores the State 3 rate. ${ }^{251}$ Temperature in this range does not appear to affect the relative sensitivity of the carrier, but more rigorous experiments are still needed.

CL binding is involved in activity, given that the CL on the ADP/ATP carrier is the negatively charged target for cationic activators, as Krämer et al. ${ }^{318}$ propose. The ADP/ATP translocase loses $50 \%$ of its activity when liver mitochondria are depleted of only $1 \%$ of their total PL by a pancreatic phospholipase $A$ with substrate specificity $\mathrm{CL}>\mathrm{PE}>\mathrm{PC}$, while Crotalus adamanteus venom phospholipase $\mathrm{A}$, with substrate specificity $\mathrm{PC}=\mathrm{PE} \gg \mathrm{CL}$, must remove 8 times more $\mathrm{PL}$ to inhibit $50 \% .{ }^{568}$ Although incorporation of the delipidated beef heart translocase protein in $\mathrm{CL}, \mathrm{PC}$ or PE vesicles activates equally, ${ }^{317} \mathrm{CL}$ binds to the isolated carrier with the unusually high molar stoichiometry of 6:1 (c.f. 2-3:1 in the cytochrome oxidase complex), and with great affinity that produces strong $\mathrm{CL}$ immobilization, inferred from ${ }^{31} \mathrm{P}$ NMR. In contrast, PC and PE are not immobilized on the protein and are easily removed; these findings indicate a specific CL-protein interaction. ${ }^{34}$ The increased mitochondrial CL/PL ratio in the hypothyroid liver may account for the abnormal thermotropic properties of the translocase, and the maintenance of high activity (decrease in $C_{i}$ ) relative to the other carriers that regulate State 3 respiration.

\section{Substrate Carriers}

Among mitochondrial substrate carriers, the 18:1-carnitine translocase has been shown to be completely CL-dependent (see Section II.J). Cardiolipins are unique in being the only membrane PL that keeps the monocarboxylate, ${ }^{418}$ dicarboxylate, ${ }^{296}$ tricarboxylate, ${ }^{580}$ $\alpha$-ketoglutarate, ${ }^{419}$ and aspartate/glutamate ${ }^{316}$ carriers active during purification and reactivates them after isolation. Only the dicarboxylate carrier has been examined and shown to be thyroid-sensitive (Table 8).

\section{Cytochrome bc $c_{1}$ Segment}

Surface spectrophotometry of intact perfused rat liver ${ }^{216}$ and studies on isolated mitochondria ${ }^{66}$ are taken to show that cytochrome $b$ is normally a control point in electron transport, although the very low $C_{\mathrm{i}}$ for the $b c_{1}$ segment (Table 8) says it is not. In hypothyroid rats, when the liver is perfused with fructose to decrease electron flow to the State 4 level by depleting $P_{i}$ and deaminating adenine nucleotides, respiratory inhibition and the degree of cytochrome $b$ oxidation are greater than normal, ${ }^{216}$ which does agree with the significant $C_{\mathrm{i}}$. In hyperthyroid rat liver, the inhibition of respiration and oxidation of cytochrome $b$ that is seen in normals does not occur. Hassinen et al. ${ }^{216}$ conclude that thyroxine loosens the coupling between phosphorylation and electron transport; conversely, hypothyroidism increases coupling in situ. In isolated rat liver mitochondria, hyperthyroidism increases the degree of reduction of cytochromes $b, c$ and $c_{1}$ during State 3 respiration (succinate, $37^{\circ} \mathrm{C}$ ), while in State 4 cytochrome $b$ is more oxidized and cytochromes $c$ and $c_{1}$ are more reduced. ${ }^{62,252}$ Mitochondrial ubiquinone content increases $75 \%$, and ubiquinone becomes more reduced; over this long treatment period, LT4 also increases concentrations of $b, c$ and $a a_{3}$. The hormone is thought to regulate State 3 respiration mainly through the $b c_{1}$ complex, and State 4 by increasing proton permeability, ${ }^{252}$ as had been shown by control strength analyses. ${ }^{618}$

The cytochrome $b c_{1}$ segment is lipid dependent. The extremely hydrophobic apocytochrome $b$ is encoded by mitochondrial DNA and synthesized in the mitochondrion, ${ }^{559}$ 
so the nascent protein assumes mature conformation at least near, if not in contact with, mitochondrial CL. Arrhenius profiles of the rate of electron transfer through $b c_{1}$ are inflected, suggesting either a conformation change in a membrane component or merely a change in the 'rate-limiting' step. ${ }^{536}$ Various PL reactivate phospholipid-depleted ubiquinol: cytochrome $c$ reductase, which contains $80 \%$ of the mitochondrial cytochrome $b$, but only in the presence of CL: CL binds, PL activate; ${ }^{59,162} \mathrm{NADH}$ : cytochrome $c$ reductase prepared from beef heart mitochondria is also CL-dependent, as judged from its inhibition by adriamycin derivatives. ${ }^{179-181}$ The $90 \%$-delipidated, inactive succinate: cytochrome $c$ reductase is reactivated by adding first ubiquinone, then asolectin. ${ }^{649}$ The residual lipids are $65 \% \mathrm{CL}, 20 \% \mathrm{PC}$ and $10 \% \mathrm{PE}$, so the 'non-specificity' of the asolectin (which itself contains $10 \%$ of total lipid $P$ as $\mathrm{CL}^{418}$ ) activation involves significant amounts of CL.

\section{Cytochrome Oxidase}

Cytochrome oxidase of bovine heart mitochondria copurifies with CL. Of the 50 lipid-binding sites on the $200 \mathrm{kDa}$ complex, 2-3 molecules of tightly bound CL are specifically required for catalytic activity of an active four-subunit fragment. Other membrane PL have a different, non-specific, activating and dispersive role, that is duplicated by detergents such as lysoPC and Tween-20. ${ }^{160.161,496}$ Based on these findings, cardiolipins are described as a prosthetic group of cytochrome oxidase involved in the cyclic transport of monovalent cations, although $\mathrm{H}^{+}$ions are omitted from the list. ${ }^{160}$ However, endogenous CL can be lowered to $0.3-0.4 \mathrm{moles} / \mathrm{mol}$ complex by replacement with dimyristoyl-PC while full oxidative activity is retained. ${ }^{484}$ EPR spectroscopy of bound spin-labeled CL shows that CL binds to cytochrome oxidase $>5$ times more strongly than this PC, but none of the binding sites on the enzyme complex are highly specific for CL. This lack of specificity may explain why cytochrome oxidase contribution to regulation of State 3 respiration in liver mitochondria is impervious to abnormal thyroid states.

\section{5. $P_{i}$ Transporter}

$P_{i}$ transporter protein purified from pig or cow heart mitochondria loses activity when $\mathrm{CL}$ is removed; only $\mathrm{CL}$ protects activity during solubilization. ${ }^{43} \mathrm{CL}$ reactivates the soluble protein, egg yolk PL activates but less. Protein-PC vesicles are inactive unless CL is added, then adriamycin inhibits. ${ }^{409,410}$ Adriamycin, an antineoplastic anthracycline aminoglycoside, forms stable intercalation complexes, probably with phosphodiester bridges of DNA and RNA (whereby it inhibits DNA replication and RNA transcription) and with the oriented phosphoester groups on the membrane-surface CL (whereby it disrupts mitochondrial structure, promotes lipid peroxidation, and inhibits oxidative phosphorylation).$^{180,415}$ In addition, adriamycin inhibits some lipogenic enzymes (isocitrate (NADP) dehydrogenase, 6-phosphogluconate dehydrogenase, and malic enzyme) by interaction with -SH groups. ${ }^{142}$ Adriamycin is thought to inhibit the $P_{i}$-transporter by making CL inaccessible. ${ }^{15}$ These observations suggest absolute CL-dependency. However, the $P_{i}$ carrier does not regulate heart mitochondrial State 3 respiration, and there is no information on thyroid effects in heart or liver mitochondria. In yeast mitochondria respiring in State 3 , the $\mathrm{P}_{\mathrm{i}}$ carrier $C_{\mathrm{i}}=0.4-0.6$, the cytochrome $a a_{3} C_{\mathrm{i}}=0.5-0.6{ }^{389}$ the $\mathrm{CL} / \mathrm{PL}$ ratio is 0.15 , but no $18: 2$ acyls are present. ${ }^{215}$ Oddly enough, $40 \mu \mathrm{M}$ LT4 accelerates yeast respiration (glucose; $37^{\circ} \mathrm{C}$ ). ${ }^{434}$

\section{Substrate Dehydrogenases}

These can apparently contribute little to the overall control of State 3 respiration in liver mitochondria of euthyroid rats $(\leqslant 0.14)$ or hormone-treated hypothyroid rats $(\leqslant 0.07)$, but they might contribute some of the 0.26 of unmeasured $C_{\mathrm{i}}$ in mitochondria from hypothyroids (Table 8). The soluble dehydrogenases that act on isocitrate, glutamate or 
malate, as well as the intrinsic membrane-bound succinate or glycerol-3-phosphate dehydrogenases, increase in thyrotoxicosis and decrease in hypothyroidism. ${ }^{291}$ The membrane-bound $\beta$-hydroxybutyrate dehydrogenase is an exception, in that thyrotoxicosis decreases its activity while hypothyroidism affects it but little. Brand and Murphy $^{51}$ believe that thyroid treatment increases de novo synthesis or alters lipid environment, or both, to increase substrate dehydrogenase activities, and that the increases in subsequent electron-transporters are less important in accelerating electron flux.

Specific PL-binding and specific PL-reactivation of a membrane-dependent enzyme are clearly distinguished in studies on the soluble apoenzyme of the $\mathrm{D}-\beta$-hydroxybutyrate dehydrogenase $\left(\mathrm{NAD}^{+}\right)$prepared from rat liver or bovine heart mitochondria. The apoenzyme specifically requires $P C$ for activity, even when in aqueous solution. ${ }^{59,152,165,288,544}$ However, although CL (or PE) itself does not reactivate, in the presence of CL 2-5 mol of pure PC per mol of enzyme half-reactivates, as compared with $60 \mathrm{~mol} \mathrm{PC} / \mathrm{mol}$ enzyme in the absence of CL. Although the apoenzyme interacts with every pure PL tested, it interacts most strongly with films containing CL. Berrez et al..$^{33}$ propose that CL molecules on the inner face govern the binding to the inner membrane. The $\beta$-hydroxybutyrate dehydrogenase is normally 'crypticized' and mostly inactive in intact mitochondria, and full expression of its activity in vitro requires membrane lysis. The similar latency of the mitochondrial inner face carnitine palmitoyltransferase $\mathrm{CPT}_{\mathrm{i}}$, and the dependency of that enzyme on CL (see Section II.J), suggest a common regulatory role for CL. Thyroidinduced depression of the $\beta$-hydroxybutyrate dehydrogenase has also been attributed to the concomitant decrease in $\left[\mathrm{NAD}^{+}\right]$, to which this enzyme is sensitive. ${ }^{341}$

Activity and amount of membrane-bound glycerol-3-phosphate dehydrogenase are remarkably thyroid-dependent (see Section II.E), but little information is on hand as to its lipid-dependency. Succinate dehydrogenase is about $30 \%$ latent in situ, and is regulated by the availability of the lipid-soluble ubiquinone and by membrane fluidity. ${ }^{22}$

Phospholipid-depleted NADH:ubiquinone reductase, which contains the NADH dehydrogenase (FMN, FeS), requires CL specifically, PL non-specifically. The NADH dehydrogenase of intact mitochondria is progressively solubilized in correlation with the course of CL hydrolysis by phospholipase A, but not with PC or PE hydrolysis, implicating $\mathrm{CL}$ in its binding into the inner membrane. ${ }^{18}$

\section{ATP-synthetase}

ATP-synthetase is thyroid- and CL-dependent. In inner membrane vesicles from liver mitochondria of hypothyroid rats, the rate of the reaction ADP $+P_{i} \rightarrow$ ATP is depressed at $30^{\circ} \mathrm{C}$ as a reflection of the linearization of the normally inflected Arrhenius profile. ${ }^{81-83,242}$ At $30^{\circ} \mathrm{C}$, respiratory rate and flavin and cytochrome contents are all normal but the ATPsynthetase, acting independently of the ADP/ATP carrier, shows a decreased $V$ and $K_{m}$ for ADP; titration of phosphorylation rate with oligomycin reveals a diminished apparent number of phosphorylating sites, enough to account for most of the phosphorylation defect. In normal inner membranes, the progressive decrease in ATPase activity during incubation with ascorbate or cysteine, which peroxidize PL and fatty acyls, correlates uniquely with the disappearance of $18: 2$ from CL ${ }^{362,525}$ Reconstitution of the ATPase of heart mitochondria (where its $C_{\mathrm{i}}$ is significant) requires lipids for the complete, oligomycinsensitive, amphipathic $F_{1} F_{0}$ assembly. $C L$, in some reports, ${ }^{102,589}$ is the most effective of the naturally available $\mathrm{PL}$, although lyso-PC is more effective. A highly purified, active ATPase that retains oligomycin-sensitivity contains $2 \mathrm{~mol}$ of CL per mol, and lesser amounts of PC and PE. ${ }^{123}$ The cardiac ATP synthetase is thyroid dependent in situ and in vivo (see Section IV.D).

\section{8. $C a^{2+}$-transporter}

The $\mathrm{Ca}^{2+}$-transporter, partially purified from cow heart submitochondrial particles, takes up very little $\mathrm{Ca}^{2+}$ when reconstituted in liposomes made of $1: 1 \mathrm{PC}+\mathrm{PE}$, but increases 
uptake linearly with [CL] added during reconstitutions, to about 12 -fold at $[\mathrm{CL}]=$ $10 \mathrm{~mol} \% .^{120}$ Liver mitochondria of hypothyroid rats pump $\mathrm{Ca}^{2+}$ ions $\left(30^{\circ} \mathrm{C}\right)$ into the matrix at rates $40 \%$ less than normals when the energy source is added ATP, but at near normal rates $(-7 \%)$ even when supported by slowed substrate oxidation. ${ }^{242,433}$ Arrhenius profiles of such ATP-driven (but not substrate-driven) transport are abnormal (J. Peabody and F. L. Hoch, unpublished data). Again these data indicate that lipid-sensitive utilization of proton flux decreases when protons emanate from the $\mathrm{H}^{+}$-ATPase and is normal (or even increased) when protons originate from substrate oxidation. When liver mitochondria from hypothyroid rats are incubated for $1 \mathrm{~min}$ with $\mathrm{T} 3$ at $37^{\circ} \mathrm{C}$, respiration in State 4 and State 3 remains unchanged, but in the presence of $\mathrm{Ca}^{2+}$ ions, increases in [T3] accelerate State 3 linearly. ${ }^{223}$ At $1 \mu \mathrm{M}$ [T3], the $V$ for $\mathrm{Ca}^{2+}$-uptake and the rate constant for $\mathrm{Ca}^{2+}$-efflux are increased maximally, suggesting that the hormone acts directly on the inner membrane to increase $\mathrm{Ca}^{2+}$ ion mobility. Thyroid-state control over $\mathrm{Ca}^{2+}$ removal from cytoplasm is more extensively studied in myocardial sarcoplasmic reticulum (see Section III.C).

\section{Pyridine Nucleotide Transhydrogenase}

As discussed in Section II.C.2, hypothyroidism doubles the $V$ of the $\sim \mathrm{TH}$ reaction but not the TH reaction in liver mitochondrial inner membrane vesicles, and T3-treatment restores normal low values of $V$. In intact liver mitochondria, the normal steady state of substrate-induced delay in reduction of intrinsic pyridine nucleotides during the State $3 \rightarrow$ State 4 transition, caused by the rate-controlling $\sim$ TH reaction, is much accelerated in hypothyroid mitochondria. ${ }^{232,234}$ Oligomycin similarly accelerates reduction in mitochondria for normal rats, ${ }^{234}$ by blocking proton egress through the ATP-synthetase and thereby stimulating TH activity even in non-phosphorylating submitochondrial particles. ${ }^{343}$ Thus, the increased proportion of inner membrane 18:2 acyls in hypothyroidism may conduct protons to the $\sim \mathrm{TH}$ and away from the ATP-synthetase.

In addition to this unusual increase in rate, the TH differs from the transporters in liver mitochondria in lipid dependence and in sensitivity to a decoupler. Energy mode of the TH reactions determines lipid dependence. ${ }^{512-516}$ The energy-independent reaction is much less inhibited by Crotalus terrificus phospholipase $\mathrm{A}_{2}$ (which is specific for PC and PE but hydrolyses little $\mathrm{CL}^{18}$ ) than is the $\sim \mathrm{TH}$ reaction in submitochondrial particles ${ }^{330,367}$ and membrane particles of Escherichia coli WS1. ${ }^{557}$ Liposomes formed from any of several PL reconstitute purified transhydrogenase from beef heart submitochondrial particles to highest TH activity; low [CL] reconstitutes most effectively, but high [CL] inhibits. ${ }^{514}$ PC is specific for the $\sim \mathrm{TH}$ mode, and small amounts of $\mathrm{CL}$ inhibit. Arrhenius profiles for $\sim \mathrm{TH}$ activity, measured in everted vesicles prepared from liver mitochondria of hypothyroid rats before or after T-treatment, maintain normal values for $E_{\mathrm{a}}$ and $T_{\mathrm{t}}$ (transition temperature). ${ }^{136,137}$ Since one protein catalyzes both $\mathrm{TH}$ and $\sim \mathrm{TH}$ reactions, these observations suggest that the lipids exert their effects via the proton leak and $\Delta p$ (as they do to regulate State 4 respiration) rather than by binding to the TH protein (as they may bind to the transporters that regulate State 3 respiration).

As noted, the 'decoupler' action of palmitate at $25^{\circ} \mathrm{C}$ does not inhibit energy-dependent TH activities in rat liver submitochondrial particles while it inhibits succinate-supported reverse electron-flow and increases State 4 respiration and ATPase activity. ${ }^{506}$ If, as Rottenberg and Hashimoto ${ }^{505,506}$ propose, the ATPase membrane-associated $\mathrm{F}_{0}$-complex serves as a 'proton capacitor' that is intermediate between oxidation and phosphorylation, and that is discharged by free fatty acids, then the lack of effect of palmitate on $\sim \mathrm{TH}$ activities indicates that a different proton pathway couples $\sim \mathrm{TH}$ to oxidation and the ATPase. If, as is discussed above, proton coupling between oxidation and phosphorylation involves 18:2 fatty acyls and CL, then the proton coupling to the $\sim \mathrm{TH}$ does not appear to involve 18:2 and CL the same way, or at all. 


\section{Repetitive Additions of $A D P$}

Recent measurements of respiration of liver mitochondria from hypothyroid rats show rates of State 4 and State 3 (succinate, $26^{\circ} \mathrm{C}$ ) that are at control levels, but both decrease progressively as ADP is added repetitively, while rates are maintained in controls. ${ }^{139}$ Hypothyroid mitochondria consume less $\mathrm{O}_{2}$ during State $4 \rightarrow 3 \rightarrow 4$ transitions. Treatment of hypothyroid rats with small doses of $\mathrm{T} 4,0.02 \mu \mathrm{g} / \mathrm{g} /$ day $\times 21$ days, reverses these abnormalities. Objections to these data include the presentation of only derivative traces, $\mathrm{d}\left[\mathrm{O}_{2}\right] / \mathrm{d} t$, rather than absolute rates, so that $\mathrm{O}_{2}$ used during proton leakage is measured indiscriminately with $\mathrm{O}_{2}$ used during phosphorylation; and the absence of added $\mathrm{Mg}^{2+}$ ions to support maximal State 3 respiration. ${ }^{552}$ Since $\Delta p$ is abnormally high in State 4 in mitochondria of thyrotoxics (Table $7 ; \Delta p$ data are needed in mitochondria from hypothyroid rats), the absence of differences in State 4 rates in the data of Ezawa et al. ${ }^{139}$ seems important. To simulate conditions in vivo, they added pulses of ADP successively. Cycling of ADP additions is recommended to 'improve' rates of State 3 respiration in normal rat liver mitochondria, ${ }^{371}$ but it beclouds physiological control mechanisms. Successive additions of ADP increase respiration in State 4 (i.e. increase proton leakage) and State 3 (i.e. redistribute $C_{\mathrm{i}}$ contributions) progressively but do not change respiratory control ratios much. Data similarly disparate with respiration before and after single additions of ADP come from ADP-cycling with liver mitochondria from EFA-deficient rats (see Section IV.E). The mechanisms do not appear to have been studied. However, the ADP/ATP carrier is electrogenic and moves protons across the inner membrane, not as part of the mechanism, but electrophoretically. ${ }^{339}$ Thus, if this carrier is involved in the respiratory effects of ADP-cycling, it may be by way of its introducing additional proton circuits. Further, multiple additions of neutralized ADP solutions introduce $\mathrm{Na}^{+}$or $\mathrm{K}^{+}$ ions, to contribute to proton movements.

\section{Liver Mitochondria of Fetal and Neonatal Rats}

Plasma [T4] and [T3] of newborn rats rise 7- to 8-fold and linearly from day 2 to day $21,{ }^{86,622}$ as shown in Table 10 . Accompanying changes in liver mitochondrial function and lipid composition should be informative on physiological and developmental effects of the hormone, as opposed to effects of hormone injections. Reports on mitochondrial respiration during the fetal and postnatal periods conflict. Some investigators find that fetal mitochondria, as compared with those from adult rats, respire (succinate, $30^{\circ} \mathrm{C}$ ) more slowly in State 3 but not in State 4..$^{11,19,206,417}$ This respiratory defect is ascribed to the low contents of adenine nucleotides, which are thought to be due to low ADP/ATP carrier activity. ${ }^{417}$ Since this carrier exchanges ADP or ATP one-for-one, some other explanation seems necessary. The proton leak in mitochondria of newborns is said to be equivalent to $7 \mathrm{pmol}$ of FCCP per $\mathrm{mg}$ protein, and in adults, to $21.5 \mathrm{pmol} / \mathrm{mg}$, but the titration curves

TABle 10. Neonatal Liver Mitochondria: Plasma [T4] and [T3]; Mitochondrial Respiration (Succinate, $25-30^{\circ} \mathrm{C}$ ) and Carbamoyl-P Synthetase Content; Liver Phospholipid Composition; and Developmental Changes

\begin{tabular}{|c|c|c|c|c|c|}
\hline & \multicolumn{4}{|c|}{ Days after birth } & \multirow[b]{2}{*}{ (Refs) } \\
\hline & $1-2$ & $4-5$ & $9-10$ & $15-21$ & \\
\hline Plasma [T4]: $(\mu \mathrm{g} / \mathrm{dl})$ & 0.6 & 1.1 & 2.5 & 3.0 & $(86,622)$ \\
\hline Plasma [T3]: (ng/dl) & 10 & 20 & 42 & 79 & $(86,622)$ \\
\hline \multirow{7}{*}{$\begin{array}{r}\text { Respiration: } \\
\text { State } 4 \text { (ng atom O/min/mg) } \\
\text { State } 3 \text { (ng atom O/min/mg) } \\
\text { Carbamoyl-P synthetase: } \\
(\% \Sigma \text { polypeptides) } \\
\text { Liver PL: } 18: 2 / \mathrm{PL}(\%) \\
18: 2 / \mathrm{CL}(\%) \\
\mathrm{CL} / \mathrm{PL}(\%)\end{array}$} & & & & & \\
\hline & 53 & 30 & 26 & 25 & $(402,480)$ \\
\hline & 159 & 150 & 143 & 150 & $(402,480)$ \\
\hline & 12 & 16 & 20 & 20 & $(402)$ \\
\hline & 5.8 & 5.5 & & 10.6 & (546) \\
\hline & 43 & 47 & 45 & 78 & $(402,640)$ \\
\hline & 6.0 & 8.8 & 9.9 & 12.2 & $(402)$ \\
\hline
\end{tabular}


are not linear for the newborns, and the respiratory rates at $[\mathrm{FCCP}]=0$ (i.e. State 4) are similar to those in adults. ${ }^{19}$ Comparison of methods for isolating mitochondria shows that the presence of bovine serum albumin greatly improves the poor recovery of fetal (but not adult) mitochondria over that in conventional media containing sucrose-EDTA, ${ }^{480}$ which suggests that conventional methods selectively damage fetal mitochondria. With albumin or mannitol in the isolating media, mitochondria prepared by these methods from livers of 1-day old rats leak protons (State 4, succinate, $25-30^{\circ} \mathrm{C}$ ) $70-100 \%$ faster than do mitochondria from adult rats and are permeable to low molecular-weight solutes, while State 3 rates are at adult levels ${ }^{402,480}$ (Table 10). State 4 respiration halves between day 2 and 9, while State 3 respiration stays constant. In spite of this leakiness, such fetal mitochondria phosphorylate ADP efficiently ${ }^{480}$ and the ADP/O ratio stays at 2.0 after birth. ${ }^{402}$ Thus, the proton leak correlates inversely with the increasing plasma [T3] and [T4]-the opposite of the direct correlation seen when adult thyroid state is altered. In newborns, the ATP synthetase $\left(C_{\mathrm{i}}=0.6\right)$, the $\mathrm{P}_{\mathrm{i}}$ carrier $\left(C_{\mathrm{i}} \leqslant 0.13\right)$, the dicarboxylate carrier $\left(C_{\mathrm{i}}=0.12\right)$ and the ADP/ATP carrier $\left(C_{\mathrm{i}}=0.15\right)$ regulate State $3 ;{ }^{19}$ the first two do not regulate in adult liver mitochondria.

Liver phospholipids of 1-day rats contain $5.8 \% 18: 2$, which doubles between days 5 and $15 .^{546}$ The 18:2 deficit in neonates reflects in part the amount and composition of mitochondria: the $18: 2$ content of $C L$ is $26 \%$ lower in fetal livers than in maternal livers 640 and rises $31 \%$ between days 5 and $15 ;^{546}$ the low ratio of CL/ $\Sigma$ PL in the newborn liver further contributes to the low $18: 2$ contents in liver lipids, and increases linearly from day 1 to $15^{402}$ (see Ref. 106). However, because mitochondrial $\mathrm{CL} / \mathrm{mg}$ protein does not change, it appears that more mitochondria are made during early development. ${ }^{276}$ Thus, the amounts of 18:2 acyls and of CL correlate inversely with the proton leak, as is seen in adults. Relative amounts of the mitochondrial $165 \mathrm{kDa}$ carbamoyl-phosphate synthetase increase $67 \%$ from days $1-10 ; ;^{90,402}$ this enzyme is induced during the postnatal rise in plasma thyroid hormones much as it is during spontaneous Anuran metamorphosis, ${ }^{16,587}$ and so serves as a marker for gene expression induced by spontaneously rising thyroid hormone levels. Other enzymes are also induced in this period: activities of $\beta$-hydroxybutyrate, succinate, glutamate, malate, and NADH dehydogenases increase 4- to 20-fold in homogenates, less in mitochondria (further showing mitochondrial replication). ${ }^{206,276,627}$ Mitochondrial glycerol-3-phosphate dehydrogenase activity does not increase, ${ }^{206}$ indicating that thyroid hormones are not omnipotent in inducing hepatic enzymes during postnatal development.

Thyroid levels do not affect liver PL contents of 18:2 acyls and CL/PL ratios in newborns like they do in adults. The effect of thyroid in newborns may be more on the incorporation of 18:2 into liver PL and especially CL (Table 2) than on the depletion of 18:2 acyls by further desaturation as it is in adults. Fetal storage of maternal 18:2 is limited, and CL 18:2 is low. ${ }^{640}$ EFA-deficiency may produce the rapid mitochondrial proton leak (see Section IV.E) and subsequent suckling may supply 18:2 while 18:2-incorporating enzymes develop under thyroid and insulin ${ }^{158}$ influences. Interestingly, the 18:2 content of PC and PE fractions of fetal livers are at adult levels, but their contents of $16: 1$ and $18: 1$ are 2.5 to 4 times above contents in the dam. ${ }^{275}$ The greater contribution of mono-unsaturates to overall unsaturation resembles that seen in tadpole livers as compared with frog livers, and decreases as rapidly after injecting the tadpole with LT3 as the induction of the enzymes synthesizing urea (Ref. 238; see Section II.I).

\section{Heart and Brain Mitochondria}

Thyrotoxicosis has been said to make heart mitochondria inefficient more readily than liver mitochondria, ${ }^{39}$ but evidence to the contrary is in Table 6 . The decreased economy of isometric contraction observed in rabbits treated with LT4, $0.2 \mu \mathrm{g} / \mathrm{g} /$ day $\times 14$ days, is not related to decreased mitochondrial resynthesis of ATP.9.10 Calorimetry shows that the ratio [recovery heat liberated during diastole]/[initial heat liberated during contraction and 
relaxation] decreases, and this is mostly accounted for by an abnormal accumulation of the $V_{1}$ myosin isoprotein, which develops isometric force less efficiently for structural reasons. On the other hand, saturation transfer ${ }^{31} \mathrm{P}-\mathrm{NMR}$ studies demonstrate that perfused hearts of euthyroid rats injected with LT4, $0.35 \mu \mathrm{g} / \mathrm{g} /$ day $\times 10-14$ days, respire more rapidly but do not increase ATP synthesis flux: ${ }^{304}$ the ATP synthetase is thyroiddependent in situ and in vivo. These findings confirm that excess thyroid hormone uncouples when measured by 'non-invasive' methods, as had been suggested in hyperthyroid muscle, liver and kidney by decreased ${ }^{32} \mathrm{P}$-uptake into ATP in vivo ${ }^{281}$ (see Ref. 226). In contrast, ${ }^{31} \mathrm{P}-\mathrm{NMR}$ measurements on hearts of hypothyroid rats show a slowed ATP synthesis that is accounted for by decreased respiratory rate, not uncoupling. ${ }^{547 a}$

Isolated heart mitochondria require a greater LT3 or LT4 dose-time treatment than do liver mitochondria to achieve comparable increases in State 4 respiration ${ }^{474}$ and to deplete 18:2 (Table 6). Heart mitochondria from our thyrotoxic rats, prepared by treating normal animals rather briefly with $\mathrm{LT} 3,1 \mu \mathrm{g} / \mathrm{g} /$ day for 3 days, are not uncoupled or loose-coupled, contain normal amounts of $18: 2$ and $C L$ in $\Sigma L$, but lose State 3 respiration on standing at $4^{\circ} \mathrm{C}$ for a reason not revealed by the lipid analyses done. ${ }^{237}$ Such increased 'fragility' might be connected with the mitochondrial swelling that occurs in hypotonic sucrose solutions together with activation of endogenous phospholipase and hydrolysis of CL and $\mathrm{PC}^{297}$ or with effects like those in glucagon-treated rats, where liver mitochondria tend to lose PC, PE, and CL during preparation. ${ }^{555}$ It takes 44-60 daily hormone injections to produce heart mitochondria that are uncoupled in vitro and half-depleted of their 18:2 acyls (Table 6).

In hypothyroid rats, heart mitochondrial State 4 respiration (glutamate) is at normal levels at $32^{\circ} \mathrm{C} .{ }^{550}$ Injecting hypothyroid rats once with LT3, $0.25 \mu \mathrm{g} / \mathrm{g}$, increases State 4 rate by $80 \%$ in 1 day and maintains an elevated rate for at least 3 days. State 3 respiration responds to altered temperatures abnormally: rates are below normal only when measured at temperatures between $20^{\circ} \mathrm{C}$ and $34^{\circ} \mathrm{C}$, reflecting the fact that Arrhenius profiles of hypothyroid preparations are normal below about $18^{\circ} \mathrm{C}$, but show an abnormally high $E_{\mathrm{a}}$ above that temperature. The mitochondrial $\Sigma \mathrm{L}$ and PL contents are normal, as are the proportions of PC, PE, PS, and CL (c.f. the $72 \%$ increase in CL/PL in liver mitochondria). The 18:2 content in hypothyroid PL is $35.5 \%$ (normals, $26.8 \%$ ), due to rises in 18:2 content of PC and PE, but not in CL.

Heart and liver mitochondria have different lipid compositions, carrier protein contents, and regulatory mechanisms, ${ }^{117,216}$ which may account for the differences in thyroid effects. Although the heart has less apparatus for lipid syntheses, heart mitochondria contain twice as much $C L$ per $\Sigma$ PL as liver mitochondria, and the CL contains about $50 \%$ more 18:2. Fasting/refeeding depletes the 18:2 acyls in heart mitochondria much less than in liver mitochondria ${ }^{7,8}$ (Table 5). Heart mitochondria contain twice as much ATP synthetase and 4 times as much ADP/ATP carrier. Their State 3 respiration (pyruvate/malate, $25^{\circ} \mathrm{C}$ ) is regulated by the ATP synthetase $\left(C_{\mathrm{i}}=0.46\right)$ and the NADH dehydrogenase $\left(C_{\mathrm{i}}=0.37\right)$, and not at all by the ADP/ATP carrier ${ }^{117}$-all in contrast to the roles of these component steps in liver mitochondria (Table 8).

If mitochondrial 18:2 acyls and/or CL mediate some thyroid actions, as they may in liver mitochondria and less in heart mitochondria, then brain mitochondria offer another system to test the generality. Mature brain mitochondrial function is completely insensitive to thyroid status. ${ }^{564}$ Brain mitochondrial $\mathrm{CL} / \mathrm{PL}$ ratios are 2-5\%, and CL 18:2 acyl content is only $10 \%$; PC contains no $18: 2$, PE contains $1 \% 18: 2 .{ }^{632}$ Fasting/refeeding fails to increase the activities of lipogenic enzymes in brain (see Section II). Essential fatty acid (EFA) deficiency depletes tissue 18:2 in the order liver $>$ heart $>$ brain, and depletes brain $20: 4$ little, if at all. Thus, the insensitivity of brain mitochondria to thyroid status is in contrast to the low sensitivity of heart mitochondria, which contain lots of CL with lots of $18: 2$ in it, both of which are hard to deplete. However, mitochondria in immature brain are quite thyroid-responsive, ${ }^{564}$ but contain no $\mathrm{CL}$ : as in neonatal liver mitochondria, the thyroid-induced development of an adult complement of enzymes and transporters seems to predominate over thyroid effects mediated by membrane lipids. 


\section{E. Essential Fatty Acid Deficiency}

Essential fatty acid metabolism and the thyroid state of animals are related: the BMR increases within 1-2 weeks (the earliest sign) in animals on a fat-free diet, and feeding small amounts of methyl linoleate corrects it within days; ${ }^{360.630}$ the hypermetabolism is not accompanied by thyroid hypersecretion; ${ }^{408}$ dietary linoleic acid protects against experimental thyrotoxicity, ${ }^{134}$ and Klein and Johnson ${ }^{310}$ noted that fat deficiency and hyperthyroidism each uncouple oxidative phosphorylation in a mechanism that might involve mitochondrial unsaturated fatty acids.

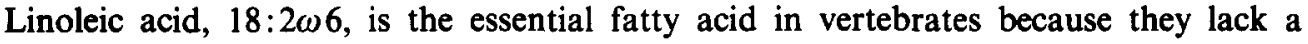
$\Delta$ 12-desaturase; its 20:4 06 derivative is absent from most lab chow diets for rats. Considerable evidence contradicts the view ${ }^{207}$ that $\omega 6$-PUFA are essential as precursors of prostanoids but not for the function of any membrane. Daily EFA requirements are orders of magnitude greater than production of prostaglandins; prostaglandin synthetase inhibitors do not induce EFA-deficiency; administration of PG does not relieve all the symptoms of EFA-deficiency; ; $^{24.250}$ those symptoms are not usually associated with actions of PG; ${ }^{394,495}$ columbinic acid cures EFA-deficiency but is not a PG precursor and even inhibits the primary enzyme for PG synthesis. Columbinic acyls replace 20:4 acyls in tissues, and restore the decreased rate of ATP synthesis in heart mitochondria ${ }^{256,258}$ - but State 4 respiration rates are not mentioned.

Problems with the measurements of mitochondrial function in EFA-deficiency include the following. Different essential fatty acids have different functions ${ }^{495}$ Individual tissues and their individual PL fatty acyls respond differently to EFA-deficiency and replacement. ${ }^{422}$ Different temperatures of measurement may produce conflicting results, since membrane PUFA distribution affects Arrhenius profiles. ${ }^{489}$ Respiratory control ratios are sometimes presented without mention of the rate of State 4 respiration whereby to estimate the proton leak. Respiratory rates or control ratios presented after ADP-cycling ${ }^{44,393,508}$ are similar in controls and EFA-deficient animals; see Section IV.B.10 for objections to this procedure.

Protein-free vesicles formed from natural purified PCs are as impermeable to trapped $\mathrm{Na}^{+405}$ and $\mathrm{K}^{+465}$ as are erythrocyte membranes, since $E_{\mathrm{a}}$ for diffusion is $15-17 \mathrm{kcal} / \mathrm{g}$ ion in all. Phosphatidylcholines purified from livers, kidneys and hearts of rats fed a fat-free diet for months are severely depleted of 18:2 (-82\%) and 20:4 (-90\%) acyls, and form vesicles that leak $\mathrm{Na}^{+}$, the $E_{\mathrm{a}}$ being $4.5 \mathrm{kcal} / \mathrm{g}$ ion-about the value for free ion diffusion in water. Proton diffusion in such membranes, as well as $\Delta p$ and proton leakage rates in mitochondria of EFA-deficient rats, have not, to my knowledge, been measured as yet.

Several dietary regimens to induce EFA-deficiency deplete mitochondrial 18:2 (especially in CL) and 20:4 acyls (Table 11). Feeding completely saturated fatty acids accentuates 18:2 $\omega 6$ fatty acid deficiency. Feeding diets rich in saturated fatty acids decreases the ratio of $\omega 6 / \omega 3$ PUFA in PL of mitochondria from rat liver, heart, or kidney, but leaves the ratio of saturated/unsaturated fatty acyls unchanged, suggesting a "homeostatic' mechanism to Gibson et al. ${ }^{172}$ Such homeostasis, like the increased $\omega 9$-unsaturation, may provide membrane fluidity for some nonspecific systems and prevent lethal outcome of EFA-deficiency. But only sufficient $\omega 6$-PUFA prevent appearance of abnormal mitochondrial oxidative phosphorylation and membrane properties.

When State 4 respiration is reported, EFA-deficiency almost always accelerates State 4. State 3 respiration and respiratory control ratios almost always decrease. Phosphorylative efficiency usually decreases only slightly, indicating that EFA-deficiency produces loose coupling ${ }^{342}$ or decoupling. ${ }^{506}$ Alfin-Slater and Aftergood ${ }^{6}$ concluded that defective oxidative phosphorylation is an early lesion in EFA-deficiency. One group ${ }^{283,574,636}$ reports no abnormalities in mitochondrial oxidative phosphorylation, but shows only respiratory control ratios and no absolute values for State 4 or State 3 respiratory rates. Mitochondrial $\omega 6-P U F A$ contents in these experiments are greatly decreased, and in correlation the lipid domains are tighter packed and allow less motional freedom of spin labels and a slower frequency of volume oscillations during energy-dependent ion transport. ${ }^{636}$ Some research- 
Table 11. Essential Fatty Acid Deficiency: Changes in Oxidative Phosphorylation and Mitochondrial $\% 18: 2$ Content after Feeding Diets Deficient in 18:2 for 4-14 Weeks; Controls Received Diets High in 18:2

\begin{tabular}{|c|c|c|c|c|c|c|c|c|}
\hline \multirow{2}{*}{$\begin{array}{c}\text { Dietary } \\
\text { fatty } \\
\text { acid }\end{array}$} & \multirow[b]{2}{*}{$\begin{array}{l}\text { Tissue } \\
\text { prep. }\end{array}$} & \multirow[b]{2}{*}{$\begin{array}{c}\text { Substrate } \\
\mathbf{T}^{\circ}\end{array}$} & \multicolumn{5}{|c|}{$\Delta \%$ vs. Control } & \multirow[b]{2}{*}{ (Ref.) } \\
\hline & & & $\begin{array}{c}\text { State } \\
4\end{array}$ & $\begin{array}{c}\text { State } \\
3\end{array}$ & RCR & $\mathbf{P} / \mathrm{O}$ & $\begin{array}{c}\% 18: 2 \\
\text { Frac. }\end{array}$ & \\
\hline 1. 0 & LMito. & $\alpha$ KGlutarate $30^{\circ}$ & & 0 & & -32 & & $(310)$ \\
\hline 2. 0 & LMito. & Succinate $30^{\circ}$ & +57 & & & & & (353) \\
\hline 3. 0 & LMito. & $\alpha \mathrm{K}$ Glutarate $30^{\circ}$ & +71 & & & & & $(560)$ \\
\hline 4. 0 & LMito. & Succinate $25^{\circ}$ & & & -16 & +2 & & (274) \\
\hline 5. 0 & LMito. & Succinate $25^{\circ}$ & & -14 & & -9 & $-78 \mathrm{CL}$ & $(40)$ \\
\hline 6. Sat & LMito. & Succinate $25^{\circ}$ & & & -17 & -8 & $-85 \mathrm{CL}$ & $(636)$ \\
\hline 7. Sat & LMito. & $\alpha \mathrm{K}$ Glutarate $30^{\circ}$ & +77 & & & & & $(560)$ \\
\hline 8. Sat & HMito. & $\begin{array}{l}\text { Glutamate } 37^{\circ} \\
18: 1 \text { Carnitine } 37^{\circ}\end{array}$ & $\begin{array}{l}+42 \\
+33\end{array}$ & $\begin{array}{l}-4 \\
-9\end{array}$ & $\begin{array}{l}-32 \\
-31\end{array}$ & $\begin{array}{r}-5 \\
0\end{array}$ & $-43 \mathrm{CL}$ & (259) \\
\hline 9. Tallow $\dagger$ & LMito. & Succinate $26^{\circ}$ & -35 & -20 & +23 & +14 & $-80 \Sigma \mathbf{L}$ & (3) \\
\hline 10. Lard & HMito.* & Pyruvate $37^{\circ}$ & +9 & +8 & -1 & -3 & $-47 \Sigma L$ & (508) \\
\hline 11. $+22: 1$ & HMito. & Glutamate $37^{\circ}$ & +49 & -26 & -51 & -10 & $-23 \mathrm{CL}$ & (259) \\
\hline 12. $+22: 1$ & HMito.* & $\begin{array}{l}\text { Succinate } 37^{\circ} \\
\text { Pyruvate } 37^{\circ} \mathrm{C}\end{array}$ & $\begin{array}{r}+57 \\
+119\end{array}$ & & -2 & -29 & $-35 \mathrm{CL}$ & $(88)$ \\
\hline 13. $+22: 1 \ddagger$ & $\begin{array}{l}\text { LMito. } \\
\text { HMito. }\end{array}$ & Glutamate $25^{\circ}$ & $\begin{array}{l}+17 \\
-30\end{array}$ & $\begin{array}{r}+8 \\
-60\end{array}$ & $\begin{array}{r}-7 \\
-54\end{array}$ & $\begin{array}{l}+7 \\
-7\end{array}$ & & $(257)$ \\
\hline 14. 0 & Rat & BMR & +25 & & & & & $(630)$ \\
\hline 15. 0 & Rat & BMR & +9 & & & & & (113) \\
\hline 16. 0 & Rat & BMR & +61 & & & & & (464) \\
\hline 17. 0 & $\begin{array}{l}\text { LSlice } \\
\text { Diaphragm }\end{array}$ & $\begin{array}{l}\text { Succinate } 37^{\circ} \\
\text { Succinate } 37^{\circ}\end{array}$ & $\begin{array}{l}+17 \\
+31\end{array}$ & & & & & (113) \\
\hline 18. 0 & LSlice & $\begin{array}{l}\text { Succinate } 37^{\circ} \\
\text { Caprylate } 37^{\circ}\end{array}$ & $\begin{array}{l}+6 \\
+70\end{array}$ & & & & & $(561)$ \\
\hline $\begin{array}{l}\text { 19. } 0 \\
20.0 \\
\text { 21. Sat }\end{array}$ & $\begin{array}{l}\text { LHomog. } \\
\text { LMito. } \\
\text { LMito. }\end{array}$ & $\begin{array}{l}\text { Citrate } 37^{\circ} \\
\text { CytoOxidase } 25^{\circ} \\
\text { SuccinateDeH } 25^{\circ} \\
\beta \text { OHButyrateDeH } 25^{\circ} \\
\text { MalateDeH } 25^{\circ}\end{array}$ & $\begin{array}{r}+56 \\
+38 \\
+140 \\
+150 \\
+292\end{array}$ & & & & & $\begin{array}{c}(561) \\
(325) \\
(217,218)\end{array}$ \\
\hline
\end{tabular}

LMito = liver mitochondria; HMito = heart mitochondria; $\mathbf{R C R}=$ respiratory control ratio; $\mathrm{CL}=$ cardiolipins; $\Sigma \mathrm{L}=$ total lipids. ${ }^{*} \mathrm{ADP}$-cycled. †Contains $3.4 \% 18: 2$. $\ddagger$ Fed 3 days.

ers find biochemical and structural abnormalities in mitochondria from EFA-deficient rats and conclude that they are artifacts resulting from damage during isolation, caused by mitochondrial fragility ${ }^{282,353,561,636}$ presumably arising from the abnormal fatty acyls and PL in the membranes. A similar argument of artifact was raised against thyroid effects. ${ }^{379}$

Indirect estimations indicate altered oxidative phosphorylation and mitochondrial function in vivo and in situ in EFA-deficiency. The increased BMR so indicates, assuming that resting cells respire in State 4..$^{226,231,551}$ Respiration in intact cells of liver slices from EFA-deficient rats is said to be at control rates, ${ }^{560}$ but when endogenous respiration is subtracted the corrected values are $+17 \%$ to $70 \%$ faster than controls ${ }^{113}$ (Table 11). In liver homogenates prepared from EFA-deficient rats, the 'un-isolated' mitochondria oxidize citrate $56 \%$ faster than in homogenates from controls. ${ }^{560}$ On the other hand, some but not all animal cell lines in culture require no essential fatty acids but subsist on endogenous formation of mono-enoic acids. ${ }^{567}$ No studies on the respiration or function of mitochondria in such cell lines are available to decide if the State 4 respiratory rate and proton leak are 'normal'.

EFA-deficiency also increases activities of the mitochondrial dehydrogenases specific for succinate, $\beta$-hydroxybutyrate, isocitrate, or malate. ${ }^{217,218}$ The fact that disruption of normal mitochondrial membranes by hypo-osmotic lysis, sonication, or phospholipase A raises these activities to the levels in EFA-deficiency shows that altered membrane-dependency, not synthesis of more dehydrogenases, is responsible. Such altered dependency also appears in erythrocyte and tissue membranes of EFA-deficient animals, in the loss of normal cooperativity of ionic effectors $\left(\mathrm{Na}^{+}, \mathrm{K}^{+}, \mathrm{Mg}^{2+}, \mathrm{F}^{-}\right)$of several ATPases. ${ }^{147}$

Mitochondrial structure is reported to be normal in electron microscopic studies of liver slices from EFA-deficient rats but abnormal in the isolated mitochondria, ${ }^{561}$ supporting the 
argument for artifact. However, other electron microscope studies on tissue slices find

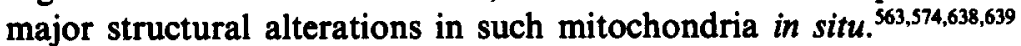

The importance, if not the essentiality, of 18:2 and its derivative fatty acyls in mitochondrial membrane function receives support from the available data on EFAdeficient animals. Crucial experimentation, such as determination of $C_{\mathrm{i}}$ values in State 3 and proton leakage in State 4 are, to my knowledge, still not reported.

\section{PERSPECTIVE}

Evidence is presented here, some of it circumstantial or incomplete, that thyroid hormone occupancy of specific receptors sets off a train of amplifying and diversifying mechanisms. A suggested sequence starts with the expression and modification of a few lipogenic enzymes; proceeds through changes in membrane phospholipid fatty acyl and cholesterol contents; with subsequent modulation of enzyme, transporter, and receptor systems, as well as unmediated membrane permeability to mono-cations; that in turn regulate concentrations of multipotent intracellular effectors. These systems include: cell membrane adenylate cyclase and intracellular cyclic nucleotide phosphodiesterases, and cell and organelle membrane $\mathrm{Ca}^{2+}$-pumps, that regulate [cAMP] and thereby protein kinases and phosphoprotein phosphatases; cell membrane $\mathrm{Na}^{+}, \mathrm{K}^{+}$-ATPase and $\mathrm{Na}^{+}-\mathrm{H}^{+}$-antiporter, that regulate intracellular $\left[\mathrm{H}^{+}\right]$and thereby gene expression and protein synthesis; phospholipases $A$ and prostaglandin synthetases; mitochondrial inner membrane permeability and transporters that regulate [ATP] and [cations]. The reduction of the diversity of thyroid hormone effects to such amplifying systems is a continuing effort.

Acknowledgements-This work was supported in part by a grant from the American Heart Association of Michigan.

(Received 22 December 1987)

\section{REFERENCES}

1. Abelin, I. and Kürsteiner, P. Biochem. Z. 198, $19-46$ (1928).

2. Abeywardena, M. Y., McMurchie, E. J., Russell, G. R., Sawyer, W. H. and Charnock, J. S. Biochim. Biophys. Acta 776, 48-59 (1984).

3. Abuirmeileh, N. M. and Elson, C. E. Lipids 15, 918-924, 925-931 (1980).

4. Akera, T., Chol, Y. R. and Yamamoto, S. In Na, K-ATPase. Structure and Kinetics, pp. 405-420 (Skou, J. C. and NorbY, J. G., eds) Academic Press, New York, 1979.

5. Alberts, A. W. and Greenspan, M. D. In New Compr. Biochem. 7 (Fatty Acid Metabolism and Its Regulation), pp. 29-58 (Numa, S., ed.), Elsevier, Amsterdam, 1984.

6. Alfin-Slater, R. B. and Aftergood, L. Physiol. Rev. 48, 758-784 (1968).

7. Allmann, D. W. and Gibson, D. M. J. Lipid Res. 6, 51-62 (1965).

8. AllmanN, D. W., Hubbard, D. D. and Gibson, D. M. J. Lipid Res. 6, 63-74 (1965).

9. Alpert, N. R. and Mulier,, L. A. Basic Res. Cardiol. 72, 153-159 (1977).

10. Alpert, N. R. and Mulieri, L. A. Fed. Proc. 45, 2597-2600 (1986).

11. Aprille, J. R. and Asimakis, G. K. Arch. Biochem. Biophys. 201, 564-575 (1980).

12. Armstrong, K. J. and Stouffer, J. E. J. Biol. Chem. 249, 4226-4231 (1974).

13. Asano, Y. Experientia (Suppl.) 32, 199-203 (1978).

14. Asano, Y., Liberman, U. A. and Edelman I. S. J. Clin. Invest. 57, 368-379 (1976).

15. Ashani, Y. and Catravas, G. N. Anal. Biochem. 109, 55-62 (1980).

16. Ashley, H., Katti, P. and Frieden, E. Dev. Biol. 17, 293-307 (1968).

17. Augenfeld, A. and Fritz, I. B. Can. J. Biochem. 48, 288-294 (1970).

18. Awasthi, Y. C., Berezney, R., Ruzicka, F. J. and Crane, F. L. Biochim. Biophys. Acta 189, $457-460$ (1969).

19. Baggetto, L., Gautheron, D. and Godinot, C. Arch. Biochem. Biophys. 232, 670-678 (1984).

20. Baneruee, S. P. and Sharma, V. K. Br. J. Pharmacol. 65, 615-621 (1979).

21. Bannett, R. R., Sampson, S. R. and Shainberg, A. Brain Res. 294, 75-82 (1984).

22. Barbero, M. C., Rial, E., Gurtubay, J. I. G., Goñi, F. M. and Macarulla, J. M. In Dev. Bioenerg. Biomemb. 6 (Structure and Function of Membrane Proteins), pp. 335-338 (Quagliarifllo, E. and Palmier, F., eds) Elsevier, Amsterdam, 1983.

23. Barker, S. B. Physiol. Rev. 31, 205-243 (1951).

24. Barker, S. B. Proc. Soc. Exp. Biol. Med. 90, 109-111 (1955).

25. Barker, S. B. and Klitganad, H. M. Am. J. Physiol. 170, 81-86 (1952).

26. Barsano, C. P. and DeGroot, L. In Molecular Basis of Thyroid Hormone Action, pp. 134-177 (OPPenheimer, J. H. and Samuels, H. H., eds) Academic Press, New York, 1983. 
27. Bartels, P. D. and Sestoft, L. Biochim. Biophys. Acta 633, 56-67 (1980).

28. Beavo, J. A., Hansen, R. S., Harrison, S. A., Hurwitz, R. L., Martins, T. J. and Mumby, M. C. Mol. Cell. Endocrinol. 28, 387-410 (1982).

29. Beg, Z. H., Stonik, J. A. and Brewer, H. B., JR. Proc. Natl. Acad. Sci. U.S.A. 75, 3678-3682 (1978).

29a. Benbrook, D. and Pfahl, M. Science 238, 788-791 (1987).

30. Benfey, B. G., Kunos, G. and Nickerson, M. Br. J. Pharmacol. 51, 253-257 (1974).

31. Bergseth, S., Lund, H., Poisson, J.-P., Bremer, J., Davis-Van Thienen, W. and Davis, E. J. Biochim. Biophys. Acta 876, 551-558 (1986).

32. Bergstrom, J. D. and Reitz, R. C. Arch. Biochem. Biophys. 204, 71-79 (1980).

33. Berrez, J. M., Pattus, F. and Latruffe, N. Arch. Biochem. Biophys. 243, 62-69 (1985).

34. Beyer, K. and Klingenberg, M. Biochemistry 24, 3821-3826 (1985).

35. Bieber, L. L., Emaus, R., Valkner, K. and Farrell, S. Fed. Proc. 41, 2858-2862 (1982).

36. BieBer, L. L. and Fiol, C. J. Biochem. Soc. Trans. 14, 674-676 (1986).

37. Bieber, L. L., Markwell, M. A. K., Blair, M. and Helmrath, T. A. Biochim. Biophys. Acta 326, $145-154$ (1973).

38. Bilheimer, D. W. Am. J. Cardiol. 57, 7H-15H (1986).

39. Bing, R. J. Am. J. Med. 30, 679-691 (1961).

40. Biran, L. A., Bartley, W., Carter, C. W. and Renshaw, A. Biochem. J. 94, 247-251 (1965).

41. Bird, M. I., Munday, L. A., Saggerson, E. D. and Clark, J. B. Biochem. J. 226, $323-330$ (1985).

42. Biron, R., Burger, A., Chinet, A., Clausen, T. and Dubois-Ferrière, R. J. Physiol. (London) 297, $47-60$ (1979)

43. Bisaccia, F., Tommasino, M. and Palmieri, F. In. Dev. Bioenerg. Biomemb. 6 (Structure and Function of Membrane Proteins), pp. 339-346, (Quagliariello, E. and Palmieri, F., eds) Elsevier, Amsterdam, 1983.

44. Bishop, P. D. and Atkinson, D. E. Arch. Biochem. Biophys. 230, 335-344 (1984).

45. Block, M. R., Pougeots, R. and Vignais, P. V. FEBS Lett. 117, 335-340 (1980)

46. Blond, D. M. and Whittam, R. Biochem. J. 92, 158-167 (1964).

47. Boardman, L., Huett, M., Lamb, J. F., Newton, J. P. and Polson, J. M. J. Physiol. (London) 241, $771-794$ (1974).

48. Bogdanovsky-Sequeval, D., Raymondjean, M., Bachner, L., Kneip, B. and Schapira, G. Life Sci. 27, 387-394 (1980).

49. Bohnensack, R., Küster, U. and Letko, G. Biochim. Biophys. Acta 680, 271-280 (1982).

50. Brady, P. S. and Brady, L. J. Fed. Proc. 46, 2005 (1987).

51. Brand, M. D. and MurPhy, M. P. Biol. Rev. 62, 141-193 (1987).

52. Brameall, J. Biochemistry 26, 2848-2855 (1987).

53. BREMER, J. Biochim. Biophys. Acta 665, 628-631 (1981).

54. BREMER, J. Physiol. Rev. 63, 1420-1480 (1983).

55. Bremer, J. and Osmundsen, H. In New Compr. Biochem. 7, (Fatty Acid Metabolism and Its Regulation), pp. 113-154 (Numa, S., ed.) Elsevier, Amsterdam, 1984.

56. Brenner, R. R. Prog. Lipid Res. 23, 69-96 (1984).

57. Bressler, R. and Friedierg, S. J. Arch. Biochem. Biophys. 104, 427-432 (1964).

58. Bressler, R. and Wittels, B. J. Clin. Invest. 45, 1326-1333 (1966).

59. Brierley, G. P., Merola, A. J. and Fleischer, S. Biochim. Biophys. Acta 64, 218-228 (1962).

60. Brindley, D. N. and Sturton, R. G. In New Compr. Biochem. 4 (Phospholipids), pp. 179-213 (Hawthorne, J. N. and Ansell, G. B., eds) Elsevier, Amsterdam, 1982.

61. Bronk, J. R. Science 141, 816-818 (1963).

62. Bronk, J. R. Science 153, 638-639 (1966).

63. Brooks, B., ArCh, J. R. S. and Newsholme, E. A. FEBS Lett. 146, 327-330 (1982).

64. Brooks, B., ArCh, J. R. S. and Newsholme, E. A. Biosci. Rept. 3, 263-267 (1983).

65. Brosnan, J. T., Kopec, B. and Fritz, I. B. J. Biol. Chem. 248, 4075-4082 (1973).

66. Brown, G. C. and Brand, M. D. Biochem. J. 225, 399-405 (1985).

67. Brown, G. C. and Brand, M. D. Biochem. J. 234, 75-81 (1986).

68. Brown, M. S. and Goldstein, J. L. Science 232, $34-47$ (1986).

69. Buccino, R. A., Spann, J. F., Jr., Pool, P. E., Sonnenblick, E. H. and Braunwald, E. J. Clin. Invest. 10, 1669-1682 (1967).

70. Buckley, G. A. and Jordan, C. C. Br. J. Pharmacol. 38, 394-398 (1970).

71. BUERgi, U. and AbBuehl, U. Metabolism 33, 326-328 (1984).

72. BusA, W. B. Curr. Top. Membr. Transp. 26, 291-305 (1986).

73. Capasso, G., Lin, J.-T., De Santo, N. G. and KinNe, R. Pflügers Arch. 403, 90-96 (1985).

74. Chait, A., Bierman, E. L. and Albers, J. J. J. Clin. Endocrinol. Metab. 48, 887-889 (1979).

75. Chambaz, J., Gulllouzo, A., Cardot, P., Pepin, D. and Bereziat, G. Biochim. Biophys. Acta 878, 310-319 (1986).

76. Chance, B. and Williams, G. R. J. Biol. Chem. 217, 409-424 (1955).

77. Chapman, D., Gómez-Fernández, J. C. and GoÑi, F. M. Trends Biochem. Sci. 7, 67-70 (1982).

78. Charnock, J. S., Almeida, A. F. and To, R. Arch. Biochem. Biophys. 167, 480-487 (1975).

79. Charnock, J. S. and Bashrord, C. L. Mol. Pharmacol. 11, 766-774 (1975).

80. Chaudhury, S., Ismail-Beigi, F., Gick, G., LeVenson, R. and Edelman, I. S. Mol. Endocrinol. 1, 83-89 (1987).

81. Chen, Y.-D. I. Fed. Proc. 34, 314 (1975).

82. Chen, Y.-D. I. and НоcH, F. L. Arch. Biochem. Biophys. 172, 741-744 (1976).

83. CHEN, Y.-D. I. and Hoch, F. L. Arch. Biochem. Biophys. 181, 470-483 (1977)

84. Cheng, S.-Y., Gong, Q.-H., Parkison, C., Robinson, E. A., Appella, E., Merlino, G. T. and Pastan, I. J. Biol. Chem. 262, 11221-11227 (1987). 
85. Cheng, S.-Y., Hasumura, S., Willingham, M. C. and Pastan, I. Proc. Natl. Acad. Sci. U.S.A. 83, 947-951 (1986).

86. Cheron, R. G., Kaplan, M. M. and Larsen, P. R. Endocrinology 106, 1405-1409 (1980).

87. Ciaraldi, T. and Marinetti, G. V. Biochem. Biophys. Res. Commun. 74, 984-991 (1977).

88. Clandinin, M. T. J. Nutr. 108, 273-281 (1978).

89. Clarke, P. R. H. and Bieber, L. L. J. Biol. Chem. 256, $9869-9873$ (1981).

90. Clarke, S. J. Biol. Chem. 251, 950-961 (1976).

91. Clausen, T. Physiol. Rev. 66, 542-580 (1986).

92. Clejan, S., Collipp, P. J. and Maddaiah, V. T. Arch. Biochem. Biophys. 203, 744-752 (1980).

93. Coleoni, A. H. and DeGroot, L. J. Endocrinology 106, 1103-1107 (1980).

94. COOK, H. W. and SPENCE, M. W. Biochim. Biophys. Acta 369, 129-141 (1974).

95. Coots, R. H. J. Lipid Res. 5, 473-476 (1964).

96. Correze, C., Auclair, R. and Nunez, J. Mol. Cell. Endocrinol. 5, 67-79 (1976).

97. Correze, C., Berriche, S., Tamayo, L. and Nunez, J. Eur. J. Biochem. 122, 387-392 (1982).

98. Correze, C., Laudat, M. H., Laudat, P. and Nunez, J. Mol. Cell. Endocrinol. 1, 309-327 (1974).

99. Correze, C., Pierre, M., Thibout, H. and Toru-Delbauffe, D. Biochem. Biophys. Res. Commun. 126, 1061-1068 (1985).

100. Correze, C., Pinell, P. and Nunez, J. FEBS Lett. 23, 87-91 (1972).

101. Crews, F. T. In Phospholipids and Cellular Regulation, Vol. 1, pp. 131-158, (Kuo, J. F., ed.) CRC Press, Boca Raton, FL, 1985.

102. Cunningham, C. C. and George, D. T. J. Biol. Chem. 250, 2036-2044 (1975).

103. Curfman, G. D., Crowley, T. J. and Smith, T. W. J. Clin. Invest. 59, 586-590 (1977).

104. Dang, A. Q., FaAs, F. H. and CARTER, W. J. Lipids 20, 897-902 (1985).

105. DAs, D. K. Arch. Biochem. Biophys. 203, 25-36 (1980).

106. Daum, G. Biochim. Biophys. Acta 822, 1-42 (1985).

107. Davis, E. J. and Davis-Van Thienen, W. I. A. Arch. Biochem. Biophys. 233, 573-581 (1984).

108. Davis, E. J. and Lumeng, L. J. Biol. Chem. 250, 2275-2282 (1975).

109. Davis, P. J. and Blas, S. D. Biochem. Biophys. Res. Commun. 99, 1073-1080 (1981).

110. Davidson, J. B. and Stanacev, N. Z. Biochem. Biophys. Res. Commun. 42, 1191-1199 (1971).

111. DeAn, W. L. and TANFoRD, C. Biochemistry 17, 1638-1690 (1978).

112. Debons, A. F. and Schwartz, I. L. J. Lipid Res. 2, 86-89 (1961).

113. Demartinis, F. D. and Paschicis, K. E. Am. J. Physiol. 199, 879-882 (1960).

114. Deykin, D. and Vaughan, M. J. Lipid Res. 4, 200-203 (1963).

115. Diamant, S., Gorin, E. and Shafrir, E. Eur. J. Biochem. 26, 553-559 (1972).

116. Dillon, R. S. and Hoch, F. L. Biochem. Med. 1, 219-229 (1967).

117. Doussiere, J., Ligeti, E., Brandolin, G. and Vignais, P. V. Biochim. Biophys. Acta 766, 492-500 (1984).

118. Dow, D. S. Biochemistry 6, 2915-2922 (1967).

119. Dow, D. S. (1967) Biochemistry 6, 3350-3355 (1967)

120. Dubinsky, W., Kandrach, A. and Racker, E. In Membrane Bioenergetics, pp. 267-280 (LEe, C.-P., Schatz, G. and ERnSTER, L., eds) Addison-Wesley, Reading, MA, 1979.

121. Dugan, R. E., Ness, G. C., Lakshmanan, M. R., Nepokroeff, C. M. and Porter, J. W. Arch. Biochem. Biophys. 161, 499-504 (1974).

122. Dugan, R. E. and Porter, J. W. In Biochemical Actions of Hormones, Vol. 4, pp. 197-247 (LiTwack, G., ed.) Academic Press, New York, 1977.

122a. Duszynski, J., Groen, A. K., Wanders, R. J. A., Vervoorn, R. C. and TAger, J. M. FebS Lett. 146, 262-266 (1982).

123. Eble, K. S. and Cunningham, C. C. Fed. Proc. 46, 1966 (1987).

124. Eckel, J., RaO, G. S., RAO, M. L. and Breuer, H. Biochem. J. 182, 473-491 (1979).

125. Edelman, I. S. N. Engl. J. Med. 290, 1303-1308 (1974).

126. Edelman, I. S., Pressley, T. H. and Hiatt, A. In The Sodium Pump: Proc. 4th Intl. Conf. on Na, $K$-ATPase, Aug. 1984, Cambridge. pp. 153-159, The Company of Biologists, Cambridge, UK, 1985.

127. Edman, J. C., Ellis, L., Blacher, R. W., Roth, R. A. and RutTer, W. J. Nature 317, 267-270 (1985).

128. Edwards, Y. N., Chase, J. F. A., Edwards, M. R. and TubBs, P. K. Eur. J. Biochem. 46, 209-215 (1974)

129. Elks, M. L. and Mangiello, V. C. Endocrinology 117, 947-953 (1985).

130. Ellefson, R. D. and Mason, H. L. Endocrinology 75, 179-186 (1964).

131. Else, P. L. and Hulbert, A. J. Am. J. Physiol. 253, R1-R7 (1987).

132. Emmelot, P., Bos, C. J. and van Hoeven, R. P. Meth. Enzymol. 31, 75-90 (1974).

133. Engelhard, V. H., Esko, J. D., Storm, D. R. and Glaser, M. Proc. Natl. Acad. Sci. U.S.A. 73, 4482-4486 (1976).

134. ERShOFF, B. H. J. Nutr. 39, 259-281 (1949).

135. Estabrook, R. W. Meth. Enzymol. 10, 41-47 (1967).

136. Evans, T. C. Ph.D. Thesis, Department of Biological Chemistry, The University of Michigan, Ann Arbor, MI, 1976.

137. Evans, T. C. and Носн, F. L. Biochem. Biophys. Res. Commun. 69, 635-640 (1976).

138. Everett, A. W., Umeda, P. K., Sinha, A. M., Rabinowitz, M. and Zak, R. Fed. Proc. 45, $2568-2572$ (1986).

139. Ezawa, I., Yamamoto, M., Kimura, S. and Ogata, E. Eur. J. Biochem. 141, 9-13 (1984).

140. FAAS, F. H. and CARTER, W. J. Biochem. J. 193, 845-852 (1981)

141. FAAS, F. H. and CARTER, W. J. Biochem. J. 207, 29-35 (1982).

142. Fabregat, I., Satrústegui, J. and Machado, A. Biochem. Med. 32, 289-295 (1984).

143. FAIN, J. N. and Rosenthal, J. W. Endocrinology 89, 1205-1211 (1971).

144. Fambrough, D. M. Cold Spring Harbor Symp. Quant. Biol. 48, (Pt. 1), 297-304 (1983). 
145. Fambrough, D. M. and Bayne, E. K. J. Biol. Chem. 258, 3926-3935 (1983).

146. Fanburg, B. L. J. Clin. Invest. 47, 2499-2506 (1968).

147. Farias, R. N., Bloj, B., Morero, R. D., Sineriz, F. and Trucco, R. E. Biochim. Biophys. Acta 415, 231-251 (1975).

148. Fernandez, J. L., Rosemblatt, M. and Hidalgo, C. Biochim. Biophys. Acta 599, 552-568 (1980).

149. Fiol, C. J. and Bieber, L. L. J. Biol. Chem. 259, 13084-13088 (1984).

150. Fischer, P. W. F. and Goodridge, A. G. Arch. Biochem. Biophys. 190, 334-355 (1978).

151. FitTs, R. H., Winder, W. W., Brooke, M. H., Kaiser, K. K. and Holloszy, J. O. Am. J. Physiol. 238, CI5-C20 (1980).

152. Fleischer, S., Mclntyre, J. O., Churchill, P., Fleer, E., and Maurer, A. In Dev. Bioenerg. Biomemb. 6 (Structure and Function of Membrane Proteins), pp. 283-300 (QUagliariello, E. and Palmieri, F., eds) Elsevier, Amsterdam, 1983.

153. Fleischer, S. and Rouser, G. J. Am. Oil Chem. Soc. 42, 588-607 (1965).

154. Folke, M. and Sestoft, L. J. Physiol. (London) 269, $407-419$ (1977).

155. Franzone, J., Natale, T., Negro, F., Curzio, M., Torrielli, M. V. and Sena, L. M. Boll. Soc. Ilal. Biol. Sper. 57, 604-609 (1981).

156. Freake, H. C., Mooradian, A. D., Schwartz, H. L. and Oppenheimer, J. H. Mol. Cell. Endocrinol. 44, 25-35 (1986).

157. French, S. W., Ihrig, T. J., Shaw, G. P., Tanaka, T. T. and Norum, M. L. Res. Commun. Chem. Pathol. Pharmacol. 2, 567-585 (1971).

158. Friedmann, N., Gellhorn, A. and Benjamin, W. Israel J. Med. Sci. 2, 677-682 (1966).

159. Fritz, I. B. Physiol. Rev. 41, 52-129 (1961).

160. FrY, M. and Green, D. E. Proc. Natl. Acad. Sci. U.S.A. 77, 6391-6395 (1980).

161. Fry, M. and Green, D. E. Biochem. Biophys. Res. Commun. 93, 1238-1246 (1980).

162. Fry, M. and Green, D. E. J. Biol. Chem. 256, 1874-1880 (1981).

163. Fukuda, H., Katsurada, A. and Iritani, N. Biochim. Biophys. Acta 921, 43-49 (1987).

164. Galo, M. G., Uñates, L. E. and Farias, R. N. J. Biol. Chem. 256, 7113-7114 (1981).

165. Gazzotti, P., BoCK, H.-G. and Fleischer, S. J. Biol. Chem. 250, 5782-5790 (1975).

166. Geering, K., Meyer, D. I., Paccolat, M.-P., Kraehendühl, J.-P. and Rossier, B. C. J. Biol. Chem. 260, 5154-5160 (1985).

167. Gellerich, F. N., Bohnensack, R. and Kunz, W. Biochim. Biophys. Acta 722, 381-391 (1983).

168. Gellhorn, A. and Benjamin, W. Biochim. Biophys. Acta 84, 167-175 (1964).

168a. Gervais, A., Busch, U., Wood, J. M. and Schwartz, A. J. Mol. Cell. Cardiol. 10, 1003-1015 (1978).

169. Gharbi-Chihi, J., Bismuth, J., Lissitzky, S. and Torresani, J. Biochim. Biophys. Acta 750, 282-290 (1983).

170. Gibson, D. M., Lyons, R. T., Scott, D. F. and Muto, Y. Adv. Enzyme Reg. 10, 187-204 (1972).

171. Gibson, K., Tichonicky, L. and KRUH, J. Mol. Cell. Biochem. 9, 79-83 (1975).

172. Gibson, R. A., McMurchie, E. J., Charnock, J. S. and KneEbone, G. M. Lipids 19, 942-951 (1984).

173. Gnon, G. V., Geelen, M. J. H., Buleveld, C., Quagliariello, E. and van den Bergh, S. G. Biochem. Biophys. Res. Commun. 128, 525-530 (1985).

174. Gold, M., ScotT, J. C. and Spitzer, J. J. Am. J. Physiol. 213, 239-244 (1967).

175. Gompertz, D. and Greenbaum, A. L. Biochim. Biophys. Acta 116, 441-459 (1966).

176. Goodman, H. M. and Bray, G. A. Am. J. Physiol. 210, 1053-1058 (1966).

177. Goodridge, A. G. In Molecular Basis of Thyroid Hormone Action, pp. 246-265. (OPpenHelmer, J. H. and Samuels, H. H., eds) Academic Press, New York, 1983.

178. Goodwin, C. D., Cooper, B. W. and Margolis, S. J. Biol. Chem. 257, 44694472 (1982).

179. GoOrmaghtigh, E., Brasseur, R. and RuYsschaert, J. M. Biochem. Biophys. Res. Commun. 104, 314-320 (1982).

180. Goormaghtigh, E., Chatelain, P., Caspers, J. and Ruysschaert, J. M. Biochim. Biophys. Acta 597, 1-14 (1980).

181. Goormaghtigh, E., Huart, P., Brasseur, R. and Ruysschaert, J. M. Biochim. Biophys. Acta 878, 83-94 (1986).

182. Gordon, E. R., Rochman, J., Arai, M. and Lieber, C. S. Science 216, 1319-1321 (1982).

183. Gordon, E. S. and Hemming, A. E. Endocrinology 34, 353-360 (1944).

184. Gordon, G. G. and Southren, A. L. Bull. N.Y. Acad. Med. 53, 241-259 (1977).

185. Goswami, A. and Rosenberg, I. N. Endocrinology 108, 1105-1108 (1981).

186. Goswama, A. and Rosenberg, I. N. J. Biol. Chem. 260, 82-85 (1985).

187. GREEN, K. and MATTY, A. J. J. Endocrinol. 28, 205-211 (1964).

188. GregG, V. A. and Edelman, I. S. Biochim. Biophys. Acta 887, 319-322 (1986).

189. GREIF, R. L. and SLOANE, D. Endocrinology 103, 1899-1902 (1978).

190. Gries, F. A., Matschinsky, F. and Wieland, O. Biochim. Biophys. Acta 56, 615-617 (1962).

191. GriLl, V. and Rosenovist, U. Acta Med. Scand. 194, 129-133 (1973).

192. Grill, V. and RosenQvist, U. Acta Endocrinol. 78, 39-43 (1975).

193. Grinstein, S., Cohen, S., Goetz, J. D., Rothstein, A., Mellors, A. and Gelfand, E. W. Curr. Top. Membr. Transp. 26, 115-134 (1986).

193a. Gruppo, J. and Menkes, J. H. Pediatr. Res. 5, 466-471 (1971).

194. Groen, A. K., Wanders, R. J. A., Westerhoff, H. V., van der Meer, R. and Tager, J. A. J. Biol. Chem. 257, 2754-2757 (1982).

195. Grossre, J. J. Cell. Physiol. 88, 117-126 (1976).

196. Gudbjarnason, S., Oskarsdottir, G., Olafsdottir, H. and Olafsson, G. Recent Adv. Siud. Card. Struct. Metab. 6, 193-203 (1975).

197. Guder, W., Nolte, I. and Wieland, O. Eur. J. Biochem. 4, 273-278 (1968).

198. Gudernatsch, J. F. Arch. Entwicklungsmech. Org. 35, 457-483 (1912). 
199. Guguen-Gullouzo, C., Tichonicky, L., Glaise, D. and KruH, J. Biol. Cell 44, 101-109 (1982).

200. Gumaa, K. A., Hothersall J. S., Greenbaum, A. L. and McLean, P. FEBS Lett. 80, $45-48$ (1977). 201. GutKNeCHT, J. J. Membr. Biol. 82, 105-112 (1984).

202. HABER, R. S. and LOEB, J. N. Endocrinology 111, 1217-1223 (1982).

203. HABER, R. S. and LOEB, J. N. Endocrinology 115, 291-297 (1984).

204. HABER, R. S. and LOEB, J. N. Endocrinology 118, 207-211 (1986).

205. Hahn, P. and Hassanali, S. Biol. Neonate 41, 1-7 (1982).

206. Hallman, M. Biochim. Biophys. Acta 253, 360-372 (1971).

207. Hansen, H. S. Trends Biochem. Sci. 11, 263-265 (1986).

208. Hanski, E., Rimon, G., Braun, S. and Levitzki, A. Dev. Cell Biol. 4, 213-216 (1979).

209. Hansson, P., Valdemarsson, S. and Nilsson-Ehle, P. Horm. Metab. Res. 15, 449-452 (1983).

210. Harano, Y., Kashiwagi, A., Komma, H., Suzuki, M., Hashimoto, T. and Shgeta, Y. FEBS Lett. 188, 267-271 (1985).

211. Hardie, D. G. In Mol. Asp. Cell. Reg. 1 (Recently Discovered Systems of Enzyme Regulation by Reversible Phosphorylation), pp. 33-62 (CoHEN, P., ed.) Elsevier, Amsterdam, 1980.

212. Hardie, G. Trends Biochem. Sci. 6, 75-77 (1981).

213. Harris, S. I., Balaban, R. S., Barrett, L. and Mandel, L. J. J. Biol. Chem. 256, 10319-10328 (1981).

214. Hashizume, K., Kobayashi, M., Miyamoto, T. and Yamauchi, K. Endocrinology 119, 1063-1070 (1986).

215. Haslam, J. M., Proudlock, J. W. and Linnane, A. W. Bioenergetics. 2, 351-370 (1971).

216. Hassinen, I. E., Ylikahri, R. H. and KähönEN, M. T. Arch. Biochem. Biophys. 147, 255-261 (1971).

217. Hayashida, T. and Portman, O. W. Arch. Biochem. Biophys. 91, 206-209 (1960).

218. Hayashida, T. and Portman, O. W. J. Nutr. 81, 103-109 (1963).

219. Hegrvary, C. Res. Commun. Chem. Pathol. Pharmacol. 17, 689-702 (1977).

220. HeINRICH, R. and RAPAPORT, T. A. Eur. J. Biochem. 42, 97-105 (1974).

221. HEINRICH, R. and RAPOPORT, S. M. Biochem. Soc. Trans. 11, 31-35 (1983).

222. Hemker, H. C. Biochim. Biophys. Acta 63, 46-54 (1962).

223. Herd, P. A. Arch. Biochem. Biophys. 188, 220-225 (1978).

224. Hiatt, A., McDonough, A. A. and Edelman, I. S. J. Biol. Chem. 259, 2629-2635 (1984).

225. Hidalgo, C., Ikemoto, N. and Gergely, J. J. Biol. Chem. 251, 4224-4232 (1976).

225a. Himms-Hagen, J. Ann. Rev. Physiol. 38, 315-351 (1976).

226. Hoch, F. L. Physiol. Rev. 42, 605-671 (1962).

227. HoCH, F. L. Endocrinology 77, 991-998 (1965).

228. Hoch, F. L. Proc. Natl. Acad. Sci. U.S.A. 58, 506-512 (1967).

229. Носн, F. L. Arch. Biochem. Biophys. 124, 238-247 (1968).

230. HocH, F. L. Arch. Biochem. Biophys. 124, 248-257 (1968).

231. HoCH, F. L. Energy Transformations in Mammals: Regulatory Mechanisms, In Physiological Chemistry, Vol. 3, 217 pp. (Masoro, E. J. ed.) Saunders, Philadelphia, PA, 1971.

232. Hoch, F. L. Arch. Biochem. Biophys. 150, 810-811 (1972).

233. Hосн, F. L. In Handbook of Physiology, Section 7, Endocrinology, Vol. 3, Thyroid, pp. $391-411$ (Greer, M. A. and Solomon, D. H., eds) American Physiological Society, Washington, DC, 1974.

234. Hoch, F. L. J. Bioenerg. Biomembr. 8, 223-238 (1976).

234a. HoCH, F. L. Arch. Biochem. Biophys. 178, 535-545 (1977).

235. HосH, F. L. In Endocrinology 1980, pp. $409-412$ (Cummings, I. A., Funder, J. W. and Mendelsohn, F. A. O., eds) Elsevier, Amsterdam, 1980.

236. HoCH, F. L. Prog. Lipid Res. 20, 225-228 (1982).

237. НоCH, F. L. J. Mol. Cell. Cardiol. 14, 81-86 (1982).

238. Hoch, F. L. In Phylogenic Aspects of Thyroid Hormone Actions, 19th Gunma Symposium on Endocrinology, pp. 139-149 (Institute of Endocrinology, Gunma University, eds.) Center for Academic Publications Japan, Tokyo, Japan, 1982.

239. Носн, F. L. In The Roots of Modern Biochemistry. Fritz Lipmann's Squiggle and its Consequences, pp. 645-655 (KLeinKauf, H., von Döhren, H. and JAENiCKE, L. ed.) De Gruyter, Berlin, 1988.

240. Hoch, F. L. and Chavis, T. R. Fed. Proc. 41, 968 (1982).

241. HoCh, F. L. and Chavis, T. R. Fed. Proc. 42, 325 (1983).

242. Hoch, F. L., Chen, Y.-D. I., Evans, T. C., Shaw, M. J. and Neymark, M. A. In Proc. 7th Intl. Thyroid Conf., Boston 1975, pp. 347-351 (RobBins, J. and BraVerman, L. E., eds) Excerpta Medica, Amsterdam, 1976.

243. Hoch, F. L., DePierre, J. W. and Ernster, L. Eur. J. Biochem. 109, 301-306 (1980).

244. HOCH, F. L. and Hö̀rer, B. J. Bioenerg. Biomembr. 13, 25-35 (1981).

245. HoCH, F. L. and LipManN, F. Fed. Proc. 12, 218 (1953).

246. HoCh, F. L. and LiPManN, F. Proc. Natl. Acad. Sci. U.S.A. 40, 909-921 (1954).

247. НоCH, F. L. and MotTA, M. V. Proc. Natl. Acad. Sci. U.S.A. 59, 118-122 (1968).

248. Hoch, F. L., Subramanian, C., Dhopeshwarkar, G. A. and Mead, J. F. Lipids 6, 328-335 (1981).

249. Holman, R. T. Prog. Chem. Fats Other Lipids 9, 275-348 (1971).

250. Holman, R. T., Johnson, S. B. and Hatch, T. F. Am. J. Clin. Nutr. 35, 617-623 (1982).

251. Holness, M., Crespo-Armas, A. and Mowbray, J. FEBS Lett. 177, 231-235 (1984).

252. Horrum, M. A., Tobin, R. B. and Ecklund, R. E. Biochem. Biophys. Res. Commun. 138, 381-386 (1986)

253. Hostetler, K. Y. In New Compr. Biochem. 4 (Phospholipids), pp. 215-261 (HAwthoRne, J. N. and ANSELl, G. B., eds) Elsevier, Amsterdam, 1982.

254. Hostetler, K. Y., VAN den Bosch, H. and Van Deenen, L. L. M. Biochim. Biophys. Acta 239, 113-119 (1971).

255. Hoult, J. R. S. and Moore, P. K. Br. J. Pharmacol. 62, 416P (1978).

256. Houtsmuller, U. M. T. Prog. Lipid Res. 20, 889-896 (1982). 
257. Houtsmuller, U. M. T., Struijk, C. B. and Van der Beek, A. Biochim. Biophys. Acta 218, $564-566$ (1970).

258. Houtsmuller, U. M. T., Van der Beek, A. and ZaAlberg, J. Lipids 4, 571-574 (1969).

259. Hsu, C. M. L. and KuMmerow, F. A. Lipids 12, 486-494 (1977).

260. HÜBSCHER, G. In Lipid Metabolism, pp. 280-370 (WakIL, S. J., ed.) Academic Press, New York, 1970.

261. Hudson, R. L. and SchultZ, S. G. Science 224, 1237-1239 (1984).

262. Hulbert, A. J., Augee, M. L. and Raison, J. K. Biochim. Biophys. Acta 455, 597-601 (1976).

263. Hülsmann, W. C., Oerlemans, M. C. and Geelhoed-Mieras, M. M. Biochem. Biophys. Res. Commun. 79, 784-788 (1977).

264. Hunter, C. F. and Rodwell, V. W. J. Lipid Res. 21, 399-405 (1980).

265. Ingebritsen, T. S. and COHEN, P. Science 221, 331-338 (1983).

266. Ingebritsen, T. S., Geelen, M. J. H., Parker, R. A., Evenson, K. J. and Gibson, D. M. J. Biol. Chem. 254, 9986-9989 (1979).

267. Ingebritsen, T. S. and Gibson, D. M. In Mol. Asp. Cell Reg. I (Recently Discovered Systems of Enzyme Regulation by Reversible Phosphorylation), pp. 63-94 (COHEN, P., ed.) Elsevier, Amsterdam, 1980.

268. Ingerritsen, T. S., Lee, H.-S., PARker, R. A. and Gibson, D. M. Biochem. Biophys. Res. Commun. 81, 1268-1277 (1978).

269. Ismail-Beigi, F. and Edelman, I. S. J. Gen. Physiol. 57, 711-722 (1971).

270. Ismall-Beigi, F. and Edelman, I. S. Am. J. Physiol. 225, 1172-1177 (1973).

271. Ismall-Beigi, F., Gick, G., Pressley, T. A., Chaudhury, S. and Edelman, I. S. Program 61st Meeting, Am. Thyroid Assoc., Sept. 10-13. 1986, Phoenix, AZ, P. T54, 1986.

272. Ismail-BeIGI, F., HABER, R. S. and LOEB, J. N. Endocrinology 119, 2527-2536 (1986).

273. Israel, Y., Videla, L. and Bernstein, J. Fed. Proc. 34, 2052-2059 (1975).

274. Ito, T. and Johnson, R. M. J. Biol. Chem. 239, 3201-3208 (1964).

275. Jahn, A., Joppe, U., Winkler, L. and Goetze, E. Acta Biol. Med. Ger. 19, 231-239 (1967).

276. Jakovcic, S., HadDock, J., GeTz, G. S., Rabinowitz, M. and SwifT, H. Biochem. J. 121, 341-347 (1971).

277. Jakovcic, S., SwifT, H. H., Gross, N. J. and Rabinowitz, M. J. Cell Biol. 77, 887-901 (1978).

278. JeFFCOAT, R. Essays Biochem. 15, 1-36 (1979).

279. Jeffcoat, R. and JAmes, A. T. In New Compr. Biochem. 7 (Fatty Acid Metabolism and Its Regulation), pp. 85-112 (Numa, S., ed.) Elsevier, Amsterdam, 1984.

280. Jeffcoat, R., Roberts, P. A., Ormesher, J. A. and James, A. T. Eur. J. Biochem. 101, 439-445 (1979).

281. Johnson, P. C., Posey, A. F., Patrick, D. R. and Caputto, R. Am. J. Physiol. 192, $279-282$ (1958).

282. Johnson, R. M. J. Nutr. 81, $411-414$ (1963).

283. Johnson, R. R., Bouchard, P., Tinoco, J. and Lyman, R. L. Biochem. J. 105, 343-350 (1967).

284. Jones, J. K., Ismall-Beigi, F. and Edelman, I. S. J. Clin. Invest. 51, 2498-2501 (1972).

285. Joshi, V. C. and Aranda, L. P. J. Biol. Chem. 254, 11779-11782 (1979).

286. Jothy, S., Bilodeau, J. L., Champsaur, H. and Simpkins, H. Biochem. J. 150, 133-135 (1975).

287. JUMP, D. B. and OPPENHEIMER, J. H. Endocrinology 117, 2259-2266 (1985).

288. Jurtshux, P., Sekuzu, I. and Green, D. E. Biochem. Biophys. Res. Commun. 6, 71-75 (1961).

289. KACSER, H. Biochem. Soc. Trans. 11, 35-40 (1983).

290. Kacser, H. and Burns, J. A. In Rate Control of Biological Processes, pp. 65-104 (Davies, D. D., ed.) Cambridge University Press, London, 1973.

291. Kadenbach, B. Biochem. Z. 344, 49-75 (1966).

292. Kadenbach, B., Mende, P., Kolbe, H. V. J., Stipani, I. and Palmieri, F. FEBS Lett. 139, $109-112$ (1982).

293. Kaiser, W. and Bygrave, F. L. Eur. J. Biochem. 11, 93-96 (1969).

294. KAKO, K. J. and LIU, M. S. FEBS Lett. 39, 243-246 (1974).

295. Kako, K. J. and Patterson, S. D. Biochem. J. 152, 313-323 (1975).

296. Kaplan, R. S. and Pedersen, P. L. J. Biol. Chem. 260, 10293-10298 (1985).

297. Kargapolov, A. V. Biokhimiya 44, 293-296 (1979); CA 90, $163410 \mathrm{f}$ (1980).

298. Kato, R., TAKahashi, A. and OMORI, Y. J. Biochem. (Tokyo) 68, 603-612 (1970).

299. KATZ, A. I. and EPSTEIN, F. H. J. Clin. Invest. 46, 1999-2011 (1967).

300. Kawashima, Y. and Kozuka, H. Biochim. Biophys. Acta 834, 118-123 (1985).

301. KeEnaN, T. W. and MorrE, D. J. Biochemistry 9, 19-25 (1970).

302. Kemp, A., JR., Groot, G. S. P. and ReITSMA, H. J. Biochim. Biophys. Acta 180, $28-34$ (1969).

303. Kempson, S., Marinetti, G. V. and Shaw, A. Biochim. Biophys. Acta 540, 320-329 (1978).

304. KeOGH, J. M., Matthews, P. M., Seymour, A.-M. and Radda, G. K. Adv. Myocardiol. 6, 299-309 (1985).

305. KeYes, W. G., WilcoX, H. G. and Heimberg, M. Metabolism 30, 135-146 (1981).

306. KiM, D. and SMITH, T. W. J. Clin. Invest. 74, 1481-1488 (1984).

307. Kimelberg, H. K. and Papahadjopoulos, D. J. Biol. Chem. 249, 1071-1080 (1974),

308. King, M. E., Stavens, B. W. and Spector, A. A. Biochemistry 16, 5280-5285 (1977).

309. Kinsella, J. L. and SACKTOR, B. Proc. Natl. Acad. Sci. U.S.A. 82, 3606-3610 (1985).

310. Klein, P. D. and Johnson, R. M. J. Biol. Chem. 211, 103-110 (1954).

311. Kleinzeller, A. and BRonNer, F. (eds) $\mathrm{Na}^{+}-\mathrm{H}^{+}$Exchange, Intracellular $\mathrm{pH}$, and Cell Function (ARONSON, P. S. and Boron, W. R., guest eds). Curr. Topics Membranes Transport 26, 315 pp., Academic Press, New York, 1986.

312. KlitgaARD, H. M. Endocrinology 78, 642-644 (1966).

313. KopeC, B. and Fritz, I. B. Can. J. Biochem. 8, 941-948 (1971).

314. KopeC, B. and Fritz, I. B. J. Biol. Chem. 248, 4069-4074 (1973).

315. Koper, J. W., Hoeben, R. C., HochstenbaCh, F. M. H., van Golde, L. M. G. and Lopes-Cardozo, M. Biochim. Biophys. Acta 887, 327-334 (1986).

316. KRÄMER, R. FEBS Lett. 176, 351-354 (1984).

317. KRÄMER, R. and KLINGENBERG, M. FEBS Lett. 119, 257-260 (1980). 
318. Krämer, R., MaYr, U., Heberger, C. and Tsompanidou, S. Biochim. Biophys. Acta 855, $201-210$ (1986).

319. Krebs, E. G. and Beavo, J. A. Ann. Rev. Biochem. 48, 923-959 (1979).

320. Kreiner, P. W., Keirns, J. J. and Bitensky, M. W. Proc. Natl. Acad. Sci. U.S.A. 70, $1785-1789$ (1973).

321. Krenning, E. P., Docter, R., Bernard, B., Visser, T. and Henneman, G. Biochim. Biophys. Acta 676, 314-320 (1981).

322. Krishna, G., Hynie, S. and Brodie, B. B. Proc. Natl. Acad. Sci. U.S.A. 59, 884-889 (1968).

323. Krishnamoorthy, G. and Hinkle, P. C. Biochemistry 23, 1640-1645 (1984).

324. KRUH, J. and Tichonicky, L. Eur. J. Biochem. 62, 109-115 (1976).

324a. Kumar, S., Das, D. K., Dorfman, A. E. and Asato, N. Arch. Biochem. Biophys. 178, $507-516$ (1977).

325. Kunkel, H. O. and Williams, J. N., JR. J. Biol. Chem. 189, 755-761 (1951).

326. KunOS, G., Mucci, L. and JAEGER, V. Life Sci. 19, $1597-1602$ (1976).

327. Kunos, G. and SzentivínYi, M. Nature 217, 1077-1078 (1968).

328. Kunos, G., Vermes-KunOS, I. and Nickerson, M. Nature 250, 779-781 (1974).

329. Kunos, G., Yong, M. S. and Nickerson, M. Nature 241, 119-120 (1973).

330. Kupriyanov, V. V. and Luzikov, V. N. FEBS Lett. 45, 267-270 (1974).

331. Kwok, C. T., Burnett, W. and Hardie, I. R. J. Lipid Res. 22, 570-579, 580-589 (1981).

332. Kwok, C. T., Pillay, S. P. and Hardie, I. R. In Cholesterol 7 $\alpha$-hydroxylase (7 $\alpha$-Monooxygenase) (FEARs, R. and SABINE, J, R., eds), in CRC Series in Enzyme Biology, pp. 89-102 (SABINE, J. R., ed.) CRC Press, Boca Raton, FL, 1986.

333. LAKER, M. E. and MAYES, P. A. Biochem. J. 196, 247-255 (1981).

334. Lakshmanan, M. R., Dugan, R. E., Nepokroeff, C. M., Ness, G. C. and Porter, J. W. Arch. Biochem. Biophys. 168, 89-95 (1975).

335. Lambeth, J. D. In Phospholipids and Cellular Regulation, Vol. 2, pp. 189-228 (Kuo, J. F., ed.) CRC Press, Boca Raton, FL, 1985.

336. Landriscina, C., Gnoni, G. V. and Quagliariello, E. Eur. J. Biochem. 71, 135-143 (1976).

337. Landriscina, C., Megli, F. M. and Quagliariello, E. Lipids 11, $61-66$ (1976).

338. LANDS, W. E. M. Biochem. Soc. Trans. 8, 25-27 (1980).

339. Lanoue, K., Mizani, S. M. and Klingenberg, M. J. Biol. Chem. 253, 191-198 (1978).

340. LaNout, K. F. and Schoolwerth, A. C. Metab. Transp. Mammalian Mitochon. Bioenerg. 9, 221-268 (1984).

341. Lakdy, H. A., Lee, Y.-P. and TAkemori, A. Ann. N.Y. Acad. Sci. 86, 506-511 (1960).

342. Lardy, H. A. and Wellman, H. J. Biol. Chem. 195, 215-224 (1952).

343. LeE, C.-P. and Ernster, L. Biochim. Biophys. Acta 81, 187-190 (1964)

344. LeE, H.-S. and Gibson, D. M. Hanguk Nonghwa Hakhoe Chi 23, 23-30 (1980); C. A. 93, 237567y (1981).

345. LeE, K.-L. and Miller, O. N. Arch. Biochem. Biophys. 120, 638-645 (1967).

346. Lee, K. S., Schwartz, A. and Burstein, R. J. Pharmacol. Exp. Therap. 129, 123-127 (1960).

347. Lee, M. P. and GeAR, A. R. L. J. Biol. Chem. 249, 7541-7549 (1974).

348. LeE, Y.-P. and LARDY, H. A. J. Biol. Chem. 240, 1427-1436 (1965).

349. Levey, G. S. Am. J. Med. 50, 413-420 (1971).

350. Levey, G. S. Biochem. Biophys. Res. Commun. 43, 108-113 (1971).

351. LeVeY, G. S. Ann. N.Y. Acad. Sci. 185, 449-457 (1971).

352. Levey, G. S. and Epstein, S. E. Biochem. Biophys. Res. Commun. 33, 990-995 (1968).

352a. LeVey, G. S., Skelton, C. L. and Epstein, S. E. Endocrinology 85, 1004-1009 (1969).

353. Levin, E., Johnson, R. M. and Alpert, S. J. Biol. Chem. 228, 15-21 (1957).

354. Limas, C. J. Am. J. Physiol. 253, H745-H752 (1978).

355. Lin, M. H. and Akera, T. J. Biol. Chem. 253, 723-726 (1978).

356. LIN, M. H., Romsos, D. R., AKerA, T. and Leveille, G. A. Experientia 35, 735-736 (1979).

357. Lo, C.-S., August, T. R., Liberman, U. A. and Edelman, I. S. J. Biol. Chem. 251, 7826-7833 (1976).

358. Lo, C.-S. and Edelman, I. S. J. Biol. Chem. 251, 7834-7840 (1976).

359. Lo, C.-S. and Lo, T. N. J. Biol. Chem. 255, 2131-2136 (1980).

360. LoEb, H. G. and Burr, G. O. J. Nutr. 33, 541-551 (1947).

361. LoH, H. H. and LAW, P. Y. Ann. Rev. Pharmacol. Toxicol. 20, 201-234 (1980).

362. López-Moratalla, N., Segovia, J. L. and Santiago, E. Rev. Espan. Fisiol. 29, 329-334 (1973).

363. Louis, S. L., Brivio-Haugland, R. P. and Williams, M. A. J. Supramol. Struct. 4, 487-496 (1976).

364. Lowe, A. G. and Jones, M. N. Trends Biochem. Sci. 9, 11-12 (1984).

365. LuND, H. Biochim. Biophys. Acta 918, 67-75 (1987).

366. Lund, H., Stakkestad, J. A. and Skrede, S. Biochim. Biophys. Acta 876, 685-687 (1986).

367. Luzikov, V. N., KupriYanov, V. V. and MAKhlis, T. A. J. Bioenerg. 4, 521-532 (1973).

368. Lyman, E. M., Dove, J. L. and Sribney, M. Can. J. Biochem. 54, 15-21 (1976).

369. LynCh, D. V. and Thompson, G. A. JR. Trends Biochem. Sci. 9, 442-445 (1984).

370. LyoNs, J. M. Cryobiology 9, 341-350 (1972).

371. Lyons, J. M., Raison, J. K. and Kumamoto, J. Meth. Enzymol. 32B, 258-262 (1974).

372. Maddaiah, V. Y., Clejan, S., Palekar, A. G. and Collipp, P. J. Arch. Biochem. Biophys. 210, 666-677 (1981).

373. Madden, T. D., Chapman, D. and Quinn, P. J. Nature 279, 538-540 (1979).

374. MADSEN, S. N. and SONNE, O. Nature 262, 793-795 (1976).

375. Maebashi, M., Kawamura, N., Sato, M., Imamura, A., Yoshinaga, K. and Suzuki, M. Metab. Clin. Exp. 26, 351-356, 357-361 (1977).

376. Magnuson, M. A., Dozin, B. and Nikodem, V. M. J. Biol. Chem. 260, 5906-5912 (1985).

376a. Malbon, C. C. and Greenberg, M. L. J. Clin. Invest. 69, 414426 (1982).

377. Malbon, C. C., LI, S. and Fain, J. N. J. Biol. Chem. 253, 8820-8825 (1978).

378. Malbon, C. C., Rapiejko, P. J. and Mangano, T. J. J. Biol. Chem. 260, 2558-2564 (1985).

379. Maley, G. F. Am. J. Physiol. 188, 35-39 (1957). 
380. Mariash, C. N., Kaiser, F. E., Schwartz, H. L., Towle, H. C. and Oppenheimer, J. L. J. Clin. Invest. 65, 1126-1134 (1980).

381. Mariash, C. N. and OpPenheimer, J. H. Mol. Cell. Endocrinol. 43, 3-13 (1985).

382. Marino, A., Fedriani, J. R., Sancho, M. J. and Macarulla, J. M. Exp. Clin. Endocrinol. 84, 245-251 (1984).

383. Martin, B. R. In Metabolic Regulation: A Molecular Approach, pp. 65-82, Blackwell, Oxford, 1987.

384. Martius, C. and Hess, B. Arch. Biochem. Biophys. 33, 486-487 (1951).

385. Martius, C. and Hess, B. Biochem. Z. 326, 191-203 (1955).

386. Martonosi, A., Donley, J. and Halpin, R. A. J. Biol. Chem. 243, 61-70 (1968).

387. Mathes, D. and Chevallier, F. Biochim. Biophys. Acta 441, 155-164 (1976).

388. Mayes, P. A. In Harper's Biochemistry, 21st Ed., pp. 218-225 (MurRay, R. K., Granner, D. K., MaYes, P. A. and Rodwell, V. W., eds) Lange, Los Altos, CA, 1988.

389. Mazat, J.-P., Jean-Bart, E., Rigoulet, M. and Gù̇rin, B. Biochim. Biophys. Acta 849, 7-15 (1986)

390. McDonough, A. In The Sodium Pump: Proc. 4th Intl. Conf. on Na, K-ATPase, Aug 1984, Cambridge, pp. 161-163. The Company of Biologists, Cambridge, 1985.

391. McDonough, A. and Schmit, C. Am. J. Physiol. 248, C247-C251 (1985).

392. McGarry, J. D. and Foster, D. W. Ann. Rev. Biochem. 49, 395-420 (1980).

393. McMurchie, E. J. and Raison, J. K. Biochim. Biophys. Acta 554, 364-374 (1979).

394. Mead, J. F., Alfin-Slater, R. B., Howton, D. R. and Popják, G. Lipids. Chemistry, Biochemistry, and Nutrition, p. 145. Plenum Press, New York, 1986.

395. Miceli, J. N. and Ferrell, W. J. Lipids 8, 722-727 (1973).

396. Michel, R. and Truchor, R. Bull. Soc. Chim. Biol. 44, 1141-1152 (1962).

397. Miettinen, T. A. J. Lab. Clin. Med. 71, 537-547 (1968).

398. Mitchell, P. and MoYle, J. Biochem. J. 104, 588-600 (1967).

399. Mitchell, P. and MOYLE, J. Biochem. J. 105, 1147-1162 (1967).

400. Mitchell, P. and Moyle, J. In Biochemistry of Mitochondria, pp. 53-74 (Slater, E. C., KaniUga, Z. and WortczaK, L., eds) Academic Press, New York, 1987.

401. Mitropoulos, K. A., Venkatesan, S. and Balasubramaniam, S. Biochim. Biophys. Acta 619, 247-257 (1980).

402. Miyahara, M., Kitazoe, Y., Hiraoka, N., Takeda, K., Watanabe, S., Sasaki, J., Okimasu, E., Osaki, Y., Yamamoto, H. and UtSUmi, K. Biol. Neonate 45, 129-141 (1984).

403. MoolenaAr, W. H., Mummery, C. L., van der SAag, P. T. and de LaAt, S. W. Cell 23, 789-798 (1981).

404. Mooradian, A. D., Schwartz, H. L., Mariash, C. N. and Oppenheimer, J. H. Endocrinology 117, 2449-2456 (1985).

405. Moore, J. L., Richardson, T. and Deluca, H. F. Chem. Phys. Lipids 3, 39-58 (1969).

406. MoOre, P. K. and Hoult, J. R. S. Nature 288, 271-273 (1980).

407. Morgan, D. W., Shahten, O., Keyes, W. G. and Heimberg, M. Endocrinology 110, $260-264$ (1982).

408. Morris, D. M., Panos, T. C., Finerty, J. C., Wall, R. L. and Klein, G. F. J. Nutr. 62, 119-128 (1957).

409. Müller, M., Cheneval, D. and Carafoli, E. In Dev. Bioenerg. Biomemb. 6 (Structure and Function of Membrane Proteins), pp. 351-356 (Quagliariello, E. and PalmiERI, F., eds) Elsevier, Amsterdam, 1983.

410. Müller, M., Cheneval, D. and Carafoli, E. Eur. J. Biochem. 140, 447-452 (1984).

411. Müller, M. J., Köster, H. and Seitz, H. J. Biochim. Biophys. Acta 666, 475-481 (1981).

412. Müller, M. J. and SerTz, H. J. Biochem. Pharmacol. 33, 1579-1584 (1984).

413. Muto, Y. and Gibson, D. M. Biochem. Biophys. Res. Commun. 38, 9-15 (1970).

414. Myant, N. B. and Mitropoulos, K. A. J. Lipid Res. 18, 135-153 (1977).

415. Myers, C., GianNi, L., Zweier, J., Muindi, J., Sinha, B. K. and Eliot, H. Fed. Proc. 45, 2792-2797 (1986).

416. Nakamura, H., Rue, P. A. and DeGroot, L. J. Endocrinology 112, 1427-1433 (1983).

417. Nakazawa, T., Asami, K., Suzuki, H. and Yukawa, O. J. Biochem. (Tokyo) 73, $397-406$ (1973).

418. Nakęcz, K. A., Bolli, R., Wojtczak, L. and Azzl, A. Biochim. Biophys. Acta 851, $29-37$ (1986).

419. NakȩCz, M., Nahȩcz, K. A., Broger, C., Bolli, R., WojtczaK, L. and Azzi, A. FEBS Lett. 196, 331-336 (1986).

420. Narayan, P., Liaw, C. W. and Towle, H. C. Proc. Natl. Acad. Sci. U.S.A. 81, 4687-4691 (1984).

421. Nayler, W. G., Merrillees, N. C. R., Chipperfield, D. and Kurtz, J. B. Cardiovasc. Res. 5, 469-482 (1971).

422. Nefdleman, P., Turk, J., Jakschik, B. A., Morrison, A. R. and Lefkowitz, J. B. Ann. Rev. Biochem. 55, 69-102 (1986).

423. Neelands, P. J. and Clandinin, M. T. Biochem. J. 212, 573-583 (1983).

424. Nelson, D. R. and CoRNATZER, W. E. Endocrinology 77, 37-44 (1965).

425. Nemat-Gorgani, M. and Meisami, E. J. Neurochem. 32, 1027-1032 (1979).

426. Nervi, A. M., Peluffo, R. O., Brenner, R. R. and Leikin, A. I. Lipids 15, 263-268 (1980).

427. Ness, G. C. J. Lipid Res. 20, 1048 (1979).

428. Ness, G. C. Mol. Cell. Biochem. 53/54, 299-306 (1983).

429. Ness, G. C., Dugan, R. E., Lakshmanan, M. R., Nepokroeff, C. M. and Porter, J. W. Proc. Natl. Acad. Sci. U.S.A. 70, 3839-3842 (1973).

430. Ness, G. C., Eales, S. J., Pendleton, L. C. and Smith, M. J. Biol. Chem. 260, $12391-12393$ (1985),

431. Ness, G. C., SAmple, C. E., Smith, M., Pendleton, L. C. and Eichler, D. C. Biochem. J. 233, 167-172

432. Ness, G. C., Spindler, C. D. and Moffler, M. H. Arch. Biochem. Biophys. 197, 483-499 (1979).

433. Neymark, M. A. Ph.D. Thesis, Department of Biological Chemistry, The University of Michigan, Ann Arbor, MI, 1975.

434. NG, W. G. and MaRX, L. Endocrinology 70, 669-672 (1962). 
435. Nicholls, D. G. Eur. J. Biochem. 50, 305-315 (1974).

436. Nicholls, D. G. Bioenergetics. An Introduction to the Chemiosmotic Theory, 190 pp., Academic Press, New York, 1982.

437. Nicholls, D. G. In New Compr. Biochem. 9 (Bioenergetics), pp. $29-48$ (ERnster, L., ed.) Elsevier, Amsterdam, 1984.

438. Nicholls, P. and Wenner, C. E. Arch. Biochem. Biophys. 151, 206-215 (1972).

439. Nichols, J. W. and Deamer, D. W. Proc. Nall Acad. Sci. U.S.A. 77, 2038-2042 (1980).

440. Niemeyer, H., Crane, R. K., Kennedy, E. P. and Lipmann, F. Bol. Soc. Biol. Santiago, Chile 10, 54-58 (1953).

441. Nievel, J. G. and Robinson, N. Biochem. Soc. Trans. 4, 930-931 (1976).

442. Nishiki, K., Erecinska, M., Wilson, D. F. and CoOPER, S. Am. J. Physiol. 235, C212-C219 (1978).

443. NoËL, H. and PANDE, S. V. Eur. J. Biochem. 155, 99-102 (1986).

444. Nordstrom, J. L., Rodwell, V. W. and Mitschelen, J. J. J. Biol. Chem. 252, 8924-8934 (1977).

445. Numa, S. and TANabe, T. In New Compr. Biochem. 7 (Fatty Acid Metabolism and Its Regulation), pp. 1-28 (Numa, S., ed.) Elsevier, Amsterdam, 1984.

446. NunEz, J. Mol. Cell. Endocrinol. 37, 125-132 (1984).

446a. Obata, T., Kitagawa, S., Gong, Q.-H., Pastan, I. and Cheng, S.-Y. J. Biol. Chem. 263, 782-785 (1988).

447. Ochs, R. S., Hanson, R. W. and Hall, J., (eds) Metabolic Regulation, 244 pp., Elsevier, Amsterdam, 1985.

448. Olubadewo, J. O. and Heimberg, M. Biochem. Biophys. Res. Commun. 132, 1001-1007 (1985).

449. Olubadewo, J. O., Wilcox, H. G. and Heimberg, M. J. Biol. Chem. 259, 8857-8862 (1984).

450. Omura, T. and SATo, R. J. Biol. Chem. 239, 2370-2378 (1964).

451. OPPENHEIMER, J. H. Science 203, 971-979 (1979).

452. OPpenheimer, J. H. Ann. Int. Med. 102, 374-384 (1985).

453. Oppenheimer, J. H. and SAmuels, H. H., (eds) Molecular Basis of Thyroid Hormone Action, 498 pp., Academic Press, New York, 1983.

454. Oppenheimer, J. H., Schwartz, H. L., Mariash, C. N., Kinlaw, W. B., Wong, N. C. W. and FreAke, H. C. Endocrine Rev. 8, 288-308 (1987).

455. OPPENHRimer, J. H., SchWARTZ, H. L. and Surks, M. I. Endocrinology 95, 897-903 (1974).

455a. Orrego, H., Blake, J. E., Blendis, L. M., Compton, K. V. and Israel, Y. New Engl. J. Med. 317, 1421-1427 (1987).

456. O'Shea, P. S., Feuerstein-Thelen, S. and Azzi, A. Biochem. J. 220, 795-801 (1984).

457. Oshino, N., Imal, Y. and SATo, R. J. Biochem. (Tokyo) 69, 155-167 (1971).

458. Oshino, N. and Sato, R. Arch. Biochem. Biophys. 149, 369-377 (1972).

459. Padan, E. and Rottenderg, H. Eur. J. Biochem. 40, 431-437 (1973).

460. Page, E. and McAllister, L. P. Am. J. Cardiol. 31, 172-181 (1973).

461. Pande, S. and Murthy, M. Fed. Proc. 46, 1180 (1987).

462. Pande, S. V., Murthy, M. S. R. and NoËL, H. Biochim. Biophys. Acta 877, 223-230 (1986).

463. Pande, S. V. and Parvin, R. In Carnitine Biosynthesis, Metabolism, and Functions, pp. 143-157 (Frenkel, R. A. and McGarry, J. D., eds) Academic Press, New York, 1980.

464. Panos, T. C. and Finerty, J. C. J. Nutr. 54, 315-331 (1954).

465. Papahadjopoulos, D. and Watkins, J. C. Biochim. Biophys. Acta 135, 639-652 (1967).

466. Park, C. E., Maral, E. and MookerJea, S. Biochim. Biophys. Acta 270, 50-59 (1972).

467. Parker, R. A., Miller, S. J. and Gibson, D. M. Biochem. Biophys. Res. Commun. 125, 629-635 (1984).

468. Patton, J. F. and Platner, W. S. Proc. Soc. Exp. Biol. Med. 137, 196-201 (1971).

469. Pavlovic-Hournac, M., Delbauffe, D. and Ohayon, R. Mol. Cell. Endocrinol. 12, 255-265 (1978).

470. Pavlovic-Hournac, M., Delbauffe, D. and Ohayon, R. Proc. FEBS Meet. 1978. 54 (Cyclic Nucleotides Protein Phosphorylation Cell Regulation), pp. 263-272 (1979).

471. Peifer, J. J. J. Lipid Res. 9, 193-199 (1968).

472. Philippot, J. R., CoOper, A. G. and Wallach, D. F. H. Proc. Natl. Acad. Sci. U.S.A. 74, 956-960 (1977).

473. Philipson, K. D. and Edelman, I. S. Am. J. Physiol. 232, C202-C206 (1977).

474. Piatnek-Leunissen, D. A. and Leunissen, R. L. A. Circ. Res. 25, 171-181 (1969).

475. Pietrobon, D. and Caplan, S. R. Biochemistry 24, 5764-5776 (1985).

476. Pihlajaniem, T., Helaakoski, T., Tasanen, K., Myllylä, R., Huhtala, M.-L., Koivu, J. and KiviRIKKo, K. I. EMBO J. 6, 643-649 (1987).

477. Pitt-Rivers, R. and TAta, J. R. In The Thyroid Hormones, pp. 120-123, Pergamon, New York, 1959.

478. Platner, W. S., Patnayak, B. C. and Chaffee, R. R. J. Proc. Soc. Exp. Biol. Med. 140, 857-861 (1972).

479. Pliam, N. B. and Goldfine, I. D. Biochem. Biophys. Res. Commun. 79, 166-172 (1977).

480. Pollak, J. K. Biochem. J. 150, 477-488 (1975).

481. PoOn, R., Richards, J. M. and Clark, W. R. Biochim. Biophys. Acta 649, 58-66 (1981).

482. Poorthuis, B. J. H. M. and Hostetler, K. Y. Biochim. Biophys. Acta 431, 408-415 (1976).

483. Portnay, G. I., McClendon, F. D., Bush, J. E., Braverman, L. E. and Babior, B. M. Biochem. Biophys. Res. Commun. 55, 17-21 (1973).

484. Powell, G. L., Knowles, P. F. and Marsh, D. Biochim. Biophys. Acta 816, 191-194 (1985).

485. Preiksaitis, H. G. and Kunos, G. Life Sci. 24, 35-42 (1979).

486. Pugh, E. L. and KATES, M. Lipids 14, 159-165 (1979).

487. Qureshi, A. A., Jenik, R. A., KIM, M., LoRnitzo, F. A. and PORTER, J. W. Biochem. Biophys. Res. Commun. 66, 344-351 (1975).

488. Rahimifar, M. and Ismall-Beigi, F. Mol. Cell. Endocrinol. 6, 327-331 (1977).

489. RAlson, J. K. Bioenergetics 4, 285-309 (1973).

490. Ramsay, R. R. Fed. Proc. 46, 1013 (1987).

491. Ramsay, R. R., Derrick, J. P., Friend, A. S. and Tubbs, P. K. Biochem. J. 244, 271-278 (1987). 
492. RAo, G. S. Mol. Cell. Endocrinol. 21, 97-108 (1981).

493. Ridgway, N. D. and Dolphin, P. J. J. Lipid Res. 26, 1300-1313 (1985).

494. Rimon, G., Hanski, E., Braun, S. and Levitzki, A. Nature 276, $394-396$ (1978).

495. Rivers, J. P. W. and Frankel, T. L. Br. Med. Bull. 37, 59-64 (1981).

495a. Rivers, J. P. W., Sinclair, A. J. and CrawFord, M. A. Nature 258, 171-173 (1975).

496. Robinson, N. C., STRY, F. and TAlBert, L. Biochemistry 19, 3656-3661 (1980).

497. Rodbell, M. and Jones, A. B. J. Biol. Chem. 241, 140-142 (1966).

498. Rogers, K. S., Higains, E. S. and Grogan, W. MCL. J. Nutr. 110, 248-254 (1980).

499. Roncari, D. A. K. and Murthy, V. K. J. Biol. Chem. 250, 4134-4138 (1975).

500. Roodyn, D. B., Freeman, K. B. and Tata, J. R. Biochem. J. 94, 628-641 (1965).

501. Roques, M., Tirard, A., DeGroot, L. J. and Rapaud, J. Endocrinology 100, 967-973 (1977).

502. Rosenqvist, U. Acta Med. Scand. 192, 353-359 (1972).

503. Rosenovist, U. Mol. Cell. Endocrinol. 12, 111-117 (1978).

504. Rossignol, M., Thomas, P. and Grignon, C. Biochim. Biophys. Acta 684, 195-199 (1982).

505. Rottenberg, H. Mol. Cell Biol. 4, 47-83 (1985).

506. Rottenberg, H. and Hashimoto, K. Biochemistry 25, 1747-1753 (1986).

507. Rottenberg, H., Robertson, D. E. and Rubin, E. Biochim. Biophys. Acta 809, 1-10 (1985).

508. Royce, S. M. and Holmes, R. P. Biochim. Biophys. Acta 792, 371-375 (1984).

509. Rubalcava, B. and Rodbell, M. J. Biol. Chem. 248, 3831-3837 (1973).

510. Ruggiero, F. M., Landriscina, C., Gnoni, G. V. and Quagliariello, E. Horm. Metabol. Res. 16, 37-40 (1984).

511. Ruggiero, F. M., Landriscina, C., Gnoni, G. V. and Quagliariello, E. Lipids 19, $171-178$ (1984).

512. RYDSTröm, J. Biochim. Biophys. Acta 463, 155-184 (1977).

513. RYdSTRÖM, J. J. Biol. Chem. 254, $8611-8619$ (1979).

514. RYDSTRÖM, J. and FleisCher, S. Meth. Enzymol. 50, 811-816 (1979).

515. Rydström, J., Hoek, J. B., ERICSON, B. G. and Hundal, T. Biochim. Biophys. Acta 430, $419-425$ (1976).

516. Rydström, J., Kanner, N. and RACKer, E. Biochem. Biophys. Res. Commun. 67, $831-839$ (1975).

517. Sabine, J. R. Trends Biochem. Sci. 8, 234-236 (1983).

518. Sacktor, B. and Kinsella, J. L. Curr. Topics Membr. Transp. 26, 223-244 (1986).

519. Saggerson, E. D., Bird, M. I., Carpenter, C. A., Winter, K. A. and Wright, J. J. Biochem. J. 224, $201-206$ (1984).

520. Sample, C. E., Pendleton, L. C. and Ness, G. C. Biochemistry 26, 727-731 (1987).

521. Samuels, H. H., Perlman, A. J., RaAcka, B. M. and Stanley, F. In Molecular Basis of Thyroid Hormone Action, pp. 100-139 (OPpentrermer, J. H. and Samuels, H. H., eds) Academic Press, New York, 1983.

522. Sancho, M. J., Macarulla, J. M., Segovia, J. L. and Gonzalez, C. An. Quim. 75, 413-415 (1979).

523. Sandermann, H., JR. Biochim. Biophys. Acta 515, 209-213 (1978).

524. Sanghvi, A., Grassi, E., Warty, V., Diven, W., Wight, C. and Lester, R. Biochem. Biophys. Res. Commun. 103, 886-892 (1981).

525. Santiago, E., Lopez-Moratalla, N. and Segovia, J. L. Biochem. Biophys. Res. Commun. 53, $439-445$ (1973).

526. Sap, J., Muñoz, A., Damm, K., Goldberg, Y., Ghysdael, J., Leutz, A., Beug, H. and VENNSTRÖM, B. Nature 324, 635-640 (1986).

527. Savageau, M. A. Arch. Biochem. Biophys. 145, 612-621 (1971).

528. Savageau, M. A. Curr. Topics Cell. Reg. 6, 63-130 (1972).

529. Savageau, M. A. Biomed. Biochim. Acta 44, 839-844 (1985).

530. Scarabottolo, L., Trezzi, E., Roma, P. and Cotapano, A. L. Atherosclerosis 59, 329-333 (1986).

531. Scarpulla, R. C., Kilar, M. C. and Scarpulla, K. M. J. Biol. Chem. 261, 4660-4662 (1986).

532. SCHMit, C. and McDonough, A. Fed. Proc. 46, 361 (1987).

533. Schneider, J. W., Mercer, R. W., Caplan, M., Emanuel, J. R., Sweadner, K. J., Benz, E. J., JR and Levenson, R. Proc. Natl. Acad. Sci. U.S.A. 82, 6357-6361 (1985).

534. SCHROEDER, F. J. Membr. Biol. 68, 1-10 (1982).

535. Schuurmans Stekhoven, F. M. A. H. and Bonting, S. L. Physiol. Rev. 61, 1-76 (1981)

536. Schuurmans Stekhoven, F. M. A. H., Sani, B. P. and Sanadi, D. R. Biochim. Biophys. Acta 226, 20-32 (1971).

537. Schwartz, H. L., Trence, D., Oppenheimer, J. H., Jiang, N. S. and Jump, D. B. Endocrinology 113, 1236-1243 (1983).

538. Segal, J., BuckLey, C. and Ingbar, S. H. Endocrinology 116, 2036-2043 (1985).

539. SEGAL, J. and GoRdon, A. Endocrinology 101, 150-156 (1977).

540. Segal, J. and INGBar, S. H. J. Clin. Invest. 68, 103-110 (1981).

541. Segal, J., Schwartz, H. and Gordon, A. Endocrinology 101, 143-149 (1977).

542. Seitz, H. J., Müller, M. J. and SoBoll, S. Biochem. J. 227, 149-153 (1985).

543. Sejersted, O. M. Biochim. Biophys. Acta 717, 163-174 (1982).

544. Sekuzu, I., Jurtshuk, P. and Green, D. E. J. Biol. Chem. 238, 975-982 (1963).

545. Sellers, E. A. and You, S. S. Am. J. Physiol. 163, 81-91 (1950).

546. Sen, N. (Hulbert, A. J.) Honours Master of Science Thesis, U. Wollongong, Australia, 1986.

547. Severson, D. L. and Fletcher, T. Biochim. Biophys. Acta 675, 256-264 (1981).

547a. Seymour, A.-M. L., KeOGH, J. M. and Radda, G. K. Biochem. Soc. Trans. 11, 376-377 (1983).

548. Sharma, V. K. and BanerJee, S. P. Mol. Pharmacol. 14, 122-129 (1978).

549. Shaw, M. J. and Носн, F. L. Life Sci. 19, 1359-1364 (1976).

550. Shaw, M. J. and Hoch, F. L. J. Mol. Cell. Cardiol. 9, 749-761 (1977).

551. SheArs, S. B. J. Theor. Biol. 82, 1-13 (1980).

552. Shears, S. B. Eur. J. Biochem. 146, 233-235 (1985).

553. SheArs, S. B. and Bronk, J. R. Biochem. J. 178, 505-507 (1979). 
554. ShEARs, S. B. and Bronk, J. R. J. Bioenerg. Biomembr, 12, 379-393 (1980).

555. Siess, E. A., Kientsch-Engel, R. I. and Fahimi, F. M. Biochem. Soc. Trans. 13, 657-659 (1985).

556. Sinensky, M., Pinkerton, F., Sutherland, E. and Simon, F. R. Proc. Natl. Acad. Sci. U.S.A. 76, 4893-4897 (1979).

557. Singh, A. P. and Bragg, P. D. J. Bioenerg. 7, 175-188 (1975).

558. Sipat, A. B. and SAbine, J. R. J. Lipid Res. 20, 1049 (1979).

559. Smith, E. L., Hill, R. L., Lehman, I. R., Lefkowitz, R. J., Handler, P. and White, A. In Principles of Biochemistry: General Aspects, 7th Ed, p. 372, McGraw-Hill, New York, 1983.

560. SMItH, J. and DeLuCA, H. F. J. Nutr. 78, 416-422 (1963).

561. Smith, J. and DeLuCA, H. F. J. Cell. Biol. 21, 15-26 (1964).

562. SMith, R. L. and OLDFIELD, E. Science 225, 280-288 (1984).

562a. Smith, V. L., Brent, L. G. and Thompson, R. E. J. Lipid Res. 20, 1049 (1979).

563. SNIPES, R. L. Lab. Invest. 18, 179-189 (1968).

564. Sokoloff, L. In Thyroid Horm. Brain Dev. [Proc. Conf.], pp. 367-382 (Grave, G. D., ed.) Raven, New York, 1977.

565. Solomonson, L. P., Liepkalns, V. A. and SPeCtor, A. A. Biochemistry 15, 892-897 (1976).

566. Somjen, D., Ismall-Beigi, F. and Edelman, I. S. Am. J. Physiol. 240, E146-E154 (1981).

567. SPECTOR, A. A. and YoreK, M. A. J. Lipid Res. 26, 1015-1035 (1985).

568. Spencer, T. L., See, J. K. and Bygrave, F. L. Biochim. Biophys. Acta 423, 365-373 (1976).

569. Sperling, M. A., Ganguli, S., Voina, S., Kaptein, E. and Nicoloff, J. T. Endocrinology 107, 684-690 (1980).

570. SPReCHER, H. In Polyunsaturated Fatty Acids, pp. 1-18 (Kunau, W.-H. and Holman, R. T., eds) Am. Oil Chemists Soc., Champaign, IL, 1977.

571. Stakkestad, J. A. and Bremer, J. Biochim. Biophys. Acta 711, 90-100 (1982).

572. Stakkestad, J. A. and Bremer, J. Biochim. Biophys. Acta 750, 244-252 (1983)

573. Stanacev, N. Z., Davidson, J. B., Stuhne-SekaleC, L. and Domazet, Z. Can. J. Biochem. 51, 286-304 (1973).

574. Stancliff, R. C., Williams, M. A., Itsumi, K. and Packer, L. Arch. Biochem. Biophys. 131, $629-642$ (1969).

575. Steffen, D. G. and Platner, W. S. Am. J. Physiol. 231, 650-654 (1976).

576. Steinberg, D. Pharmacol. Rev. 18, 217-235 (1966).

577. Sterling, K. New Engl. J. Med. 300, 117-123, 173-177 (1979).

578. Sterling, K. Endocrinology 119, 292-295 (1988).

579. Stiles, G. L., Caron, M. G. and Lefkowitz, R. J. Physiol. Rev. 64, 661-743 (1984).

580. Stipani, I., Barbero, M. C. and Palmieri, F. In Dev. Bioenergy. Biomemb. 6 (Structure and Function of Membrane Proteins), pp. 369-372 (Quagliariello, E. and Palmieri, F., eds) Elsevier, Amsterdam, 1983.

581. StORCH, J. and SCHACHTER, D. Biochemistry 23, 1165-1170 (1984).

582. StORY, J. A., TePPER, S. A. and KRITChevsky, D. Biochem. Med. 10, 214-218 (1974).

583. Strubelt, O. Arch. Intl. Pharmacodyn. Ther. 176, 263-288 (1968).

584. Stumpf, D. A., Eguren, L. A. and Parks, J. K. Biochem. Med. 34, 226-229 (1985).

584a. Sugden, M. C., Watts, D. I. and Marshall, C. E. Biosci. Rep. 1, 757-764 (1981).

585. Suko, J. Biochim. Biophys. Acta 252, 324-327 (1971).

586. Sun, G. Y. and SuN, A. Y. J. Neurochem. 22, 15-18 (1974).

587. Suzuki, M., Imai, K., Ito, A., Omura, T. and Sato, R. J. Biochem. (Tokyo) 62, 447-455 (1967).

588. SuzukI, S. and SuzuKI, M. Gen. Comp. Endocrinol. 45, 74-81 (1981).

589. Swanluung, P., Frigeri, K., Ohlson, K. and Ernster, L. Biochim. Biophys. Acta 305, $519-533$ (1973).

590. SWEadner, K. J. J. Biol. Chem. 254, 6060-6067 (1979).

591. Tabachnick, I. I. A. and Bonnycastle, D. D. J. Biol. Chem. 207, 757-760 (1954).

592. Takeuchi, N., Ito, M., Uchida, K. and Yamamura, Y. Biochem. J. 148, 499-503 (1975).

593. Takeuchi, N., ITo, M. and YamamuRA, Y. Atherosclerosis 20, $481-494$ (1974).

594. Tamkun, M. M. and Fambrough, D. M. J. Biol. Chem. 261, 1009-1019 (1986).

595. Tanaka, R. and Sakamoto, T. Biochim. Biophys. Acta 193, 384-393 (1969).

596. TATA, J. R. Nature 213, 566-569 (1967).

597. TATA, J. R. Biochem. J. 104, 1-16 (1967).

598. TATA, J. R. Nature 219, 331-337 (1968).

599. TATA, J. R. Biochem. J. 116, 617-630 (1970).

600. Tata, J. R., Ernster, L., Lindberg, O., Arrhenius, E., Pedersen, J. and Hedman, R. Biochem. J. 86, 408-428 (1963).

601. TAylor, V. J. and Ragan, C. I. Biochim. Biophys. Acta 851, 49-56 (1986).

602. Tepperman, H. M. and Tepperman, J. Am. J. Physiol. 202, 401-406 (1962).

603. Tepperman, H. M. and Tepperman, J. Am. J. Physiol. 206, 357-361 (1964).

604. Tepperman, J. and Tepperman, H. M. Am. J. Physiol. 200, 1069-1073 (1961).

605. Thompson, C. C., Weingerger, C., Lebo, R. and Evans, R. M. Science 237, 1610-1614 (1987).

606. Thompson, G. A., JR. The Regulation of Membrane Lipid Metabolism, 218 pp., CRC Press, Boca Raton, FL, 1980 .

607. TishleR, P. V. and HAMmond, M. E. Enzyme 20, 349-358 (1975).

608. Towle, H. C. In Molecular Basis of Thyroid Hormone Action, pp. 179-213 (OpPENHEIMER, J. H. and Samuels, H. H., eds) Academic Press, New York, 1963.

609. TSAl, J. S. and CHEN, A. Nature 275, 138-140 (1978).

610. TSE, J., WRENN, R. W. and KUo, J. F. Endocrinology 107, 6-10 (1980).

611. Turchetto, E., Sanguinetti, F. and Rossi, C. A. Boll. Soc. Ital. Biol. Sper. 31, 1324-1326 (1955).

612. Tyson, C. A., VAnde ZANDE, H. and Green, D. E. J. Biol. Chem. 251, 1326-1332 (1976). 
613. Umeki, S. and NozawA, Y. Biochim. Biophys. Acta 835, 514-526 (1985).

614. Underwood, A. H., Emmett, J. C., Ellis, D., Glynn, S. B., Leeson, P. D., Benson, G. M., Novelli, R., Pearce, N. J. and Shah, V. P. Nature 324, 425-429 (1986).

615. van Hoeven, R. P. and Emmelot, J. J. Membr. Biol. 9, 105-126 (1972).

616. Van Inwegen, R. G., Robison, G. A., Thompson, W. J., Armstrong, K. J. and Stouffer, J. E. J. Biol. Chem. 250, 2452-2456 (1975).

617. Vasdev, S. C., Korecky, B., Rastogi, R. B., Singhal, R. L. and Kako, K. J. Can. J. Physiol. Pharmacol. 55, $1311-1319$ (1977).

618. Verhoeven, A. J., Kamer, P., Groen, A. K. and Tager, J. A. Biochem. J. 226, 183-192 (1985).

619. Vignais, P. V. and Lauquin, G. J. M. Trends Biochem. Sci. 4, 90-92 (1979).

620. VolPE, J. J. and Kishimoto, Y. J. Neurochem. 19, 737-753 (1972).

621. WaKIL, S. J., Stoops, J. K. and Joshi, V. C. Ann. Rev. Biochem. 52, 537-579 (1983).

622. Walker, P., Dubois, J. D. and Dussault, J. H. Pediatr. Res. 14, 247-249 (1980).

623. Wallach, D. F. H. (with contributions by R. Schmidt-Ullrich) Membrane Molecular Biology of Neoplastic Cells, 525 pp., Elsevier, Amsterdam, 1975.

624. Wallach, S., Bellavia, J. V., Gamponia, P. J. and Bristrim, P. J. Clin. Invest. 51, 1572-1577 (1972).

625. Walravens, Ph. and Chase, H. P. J. Neurochem. 16, 1477-1484 (1969).

626. Waring, A. J., Rottenberg, H., Ohnishi, T. and Rubin, E. Proc. Natl. Acad. Sci. U.S.A. 78, 2582-2586 (1981).

627. Warshaw, J. B. Dev. Biol. 28, 537-544 (1972).

628. Weinberger, C., Thompson, C. C., Ong, E. S., Lebo, R., Gruol, D. J. and Evans, R. M. Nature 324, 641-646 (1986).

629. WERNER, H. V. Eur. J. Biochem. 48, 45-52 (1974).

630. Wesson, L. G. and Burr, G. O. J. Biol. Chem. 91, 525-539 (1931).

631. Westerhoff, H. V., Coken, A.-M. and VAN DAM, K. Biochem. Soc. Trans. 11, 81-85 (1983).

632. White, D. A. In Form and Function of Phospholipids (ANSELl, G. B., HaWthorne, J. N. and Dawson, R. M. C., eds) BBA Library 3, pp. 441-482, 1973.

633. WhittaM, R. In Cellular Functions of Membrane Transport, pp. 139-154 (HoFrman, J. F., ed.) Prentice-Hall, Englewood Cliffs, NJ, 1963.

634. WhitTAM, R. and Blond, D. M. Biochem. J. 92, 147-158 (1964).

634a. WiEDERER, O. and LÖFFLER, G. J. Lipid Res. 28, 649-658 (1987)

635. Williams, H. J. and Barrie, S. E. Biochem. Biophys. Res. Commun. 84, 89-94 (1978).

636. Williams, M. A., Stancliff, R. C., Packer, L. and KeITH, A. D. Biochim. Biophys. Acta 267, $444-456$ (1972).

637. Williams, R. J. P. FEBS Lett. 85, 9-19 (1978)

638. Wilson, J. W. and Leduc, E. H. J. Cell. Biol. 16, 281-296 (1963).

639. Wilson, J. W. and Leduc, E. H. Natl. Cancer Inst. J. 33, 721-739 (1964).

640. Winkler, L., Buanga, N.-F. and Goetze, E. Biochim. Biophys. Acta 231, 535-536 (1971).

641. WolfF, E. C. and WolfF, J. In The Thyroid Gland, Vol. 1, pp. 237-282 (PITT-RIVER, R. and TrotTER, W. R., eds) Butterworths, Washington, DC, 1964

642. Wolterink, L. F., Cornwall, R. L., Vinson, C. and Cole, L. L. Fed. Proc. 12, $158-159$ (1953).

643. Wood, J. MCM. J. Biol. Chem. 250, 3062-3066 (1975).

644. Wykle, R. L. In Lipid Metabolism in Mammals, Vol. 1, pp. 317-366 (F. SNYder, ed.) Plenum Press, New York, 1977

645. Yates, F. E., Urquhart, J. and Herbst, A. L. Fed. Proc. 17, 174 (1958).

646. York, D. A., Bray, G. A. and Yukimura, Y. Proc. Natl. Acad. Sci. U.S.A. 75, 477-481 (1978).

647. Young, D. L. and LYNEN, F. J. Biol. Chem. 244, 377-383 (1969).

648. YU, H. L. and Burton, D. N. Arch. Biochem. Biophys. 161, 297-305 (1974).

649. Yu, L., YU, C.-A. and KING, T. E. J. Biol. Chem. 253, 2657-2663 (1978)

650. Zammit, V. A. Prog. Lipid Res. 23, 39-67 (1984).

651. ZAMmIT, V. A. Biochem. Soc. Trans. 14, 676-679 (1986)

652. Zамmit, V. A. and Easom, R. A. Biochim. Biophys. Acta 927, 223-228 (1987).

653. Zierz, S. and ENGEL, A. G. Eur. J. Biochem. 149, 207-214 (1985). 